$\underline{\text { Preprint typeset in JHEP style - HYPER VERSION }}$

arXiv:0710.5621

$\operatorname{IPPP} / 07 / 82$

$\mathrm{DCPT} / 07 / 164$

KA-TP-28-2007

\title{
Dominant next-to-leading order QCD corrections to Higgs plus three jet production in vector-boson fusion
}

\author{
Terrance Figy \\ Institute of Particle Physics Phenomenology, Durham University, South Road, Durham, \\ DH1 3LE, United Kingdom \\ E-mail: terrance.figy@durham.ac.uk \\ Vera Hankele ${ }^{a}$ and Dieter Zeppenfeld ${ }^{b}$ \\ Institut für Theoretische Physik, Universität Karlsruhe, P.O.Box 6980, 76128 \\ Karlsruhe, Germany \\ E-mail:a vera@particle.uni-karlsruhe.de, ${ }^{b}$ dieter@particle.uni-karlsruhe.de
}

\begin{abstract}
We present the calculation of the dominant next to leading order QCD corrections to Higgs boson production in association with three jets via vector boson fusion in the form of a NLO parton-level Monte Carlo program. QCD corrections to integrated cross sections are modest, while the shapes of some kinematical distributions change appreciably at NLO. Scale uncertainties are shown to be reduced at NLO for the total cross section and for distributions. We consider a central jet veto at the LHC and analyze the veto probability for typical vector boson fusion cuts. Scale uncertainties of the veto probability are sufficiently small at NLO for precise Higgs coupling measurements at the LHC.
\end{abstract}

KeYwords: Standard Model, Higgs Physics, QCD, NLO Computations. 


\section{Contents}

1. Introduction 2

2. The NLO Calculation and Approximations

3. Predictions for the LHC

4. Discussion and Conclusions 26

A. Virtual Corrections 29

B. Cross section formulas 35 


\section{Introduction}

One of the primary goals of the CERN Large Hadron Collider (LHC) is the discovery of the Higgs boson and a thorough investigation of the mechanism of electroweak (EW) symmetry breaking [1, 2]. In this context, vector-boson fusion (VBF) has emerged as a particularly interesting class of processes. Higgs boson production in VBF, i.e. the EW reaction $q q \rightarrow q q H$, where the Higgs decay products are detected in association with two tagging jets, offers a promising discovery channel [3] and, once its existence has been verified, will help to constrain the couplings of the Higgs boson to gauge bosons and fermions [4].

The observation of two forward tagging jets in Higgs production via VBF at the LHC is crucial for the suppression of backgrounds [5, 6, 0, 8, 9, 10]. In addition to forward jet tagging, the veto of any additional jet activity in the central region (central jet veto) leads to further suppression of QCD backgrounds such as $W^{+} W^{-} j j, t \bar{t} j j$, and gluon fusion $H j j$ production [8, 11]. This is due to the fact that the $t$-channel exchange of quarks or gluons tends to radiate harder and more central gluons than in the VBF case. For VBF processes, jet activity in the central region is suppressed due to color singlet exchange in the $t$-channel. For the central jet veto (CJV) proposal, events are discarded if any additional jet with a transverse momentum above a minimal value, $p_{T, v e t o}$, is found between the tagging jets [5, 12, 13, 14, 15, 16, 17].

In order to utilize the CJV for the measurement of Higgs couplings, the reduction factor, $P_{\text {surv }}$, caused by the CJV on the observable signal cross section must be precisely known. The relevant information is contained in the fraction of VBF Higgs events with at least one additional veto jet between the two tagging jets, i.e. we need to know the ratio of the 3-jet Higgs cross section, $\sigma_{j j}$, to the inclusive cross section for VBF Higgs production with two tagging jets, $\sigma_{j j}$. The survival probability for the Higgs signal is then given by $P_{\text {surv }}=1-\sigma_{j j j} / \sigma_{j j}$. Perturbative survival probabilities for the CJV have been calculated for the Higgs boson signal and background processes using LO matrix elements [8, 13]. The cross section for the VBF process $p p \rightarrow H j j j$ is proportional to $\alpha_{s}$ at LO, which leads to substantial theoretical uncertainties (scale variations of $30 \%$ or more). Even though the effect on the survival probability is mitigated by the smallness of $\sigma_{j j j} / \sigma_{j j}$ (about 0.1 to 0.2 for veto thresholds $p_{T, \text { veto }} \approx 20 \mathrm{GeV}$ ), a more reliable prediction requires a calculation of the NLO QCD corrections to the Hjjj cross section. We have performed this calculation and report on the results in this paper. We do not consider additional reductions of the survival probability due to underlying event and pile-up effects. An assessment of these effects is best performed after first LHC data have become available.

A full NLO QCD calculation of the process $p p \rightarrow H j j j X$ involves virtual corrections with hexagon diagrams and would be truly challenging. As we explain in Sec. 2, all pentagon and hexagon contributions are color suppressed by a factor $1 /\left(N^{2}-1\right)$ in an $\mathrm{SU}(\mathrm{N})$ gauge theory, and they are further suppressed by the kinematics of the VBF process. For a prediction of the survival probability of the Higgs signal at the few percent level, commensurate with the knowledge of the VBF cross section for $H j j$ production at NLO and expected experimental accuracies at the LHC, these contributions are completely negligi- 
ble. We therefore perform the calculation by systematically neglecting the gauge invariant subsets of diagrams which involve $t$-channel gluon exchange and which lead to pentagons and hexagons. Similarly, we neglect identical fermion effects for four-quark final states. In Sec. 2 we more explicitly specify and justify these approximations and we briefly describe the calculation of the LO and NLO matrix elements for $H j j j$ production.

Section 3 deals with phenomenological applications of the parton-level Monte Carlo program which we have developed. We consider the $H j j j$ cross section at NLO after typical VBF cuts and discuss the reduction of the scale dependence of relevant distributions. We show that the scale dependence of $P_{\text {surv }}$ is reduced to about $1 \%$ by including the NLO QCD corrections to the three jet cross section. Conclusions are given in Sec. 1 . Explicit formulas for the virtual corrections and for finite collinear terms from initial state radiation in gluon initiated processes are collected in two Appendices. 


\section{The NLO Calculation and Approximations}

The cross section for the leading order process $p p \rightarrow H j j j$, via $\mathrm{VBF}$, has been previously calculated as the NLO real emission correction to $H j j$ production in Refs. [18, 19, 20]. The relevant Feynman graphs are depicted in Fig. 1: one needs to consider the $\mathcal{O}\left(\alpha^{3} \alpha_{s}\right)$ subprocesses $q Q \rightarrow q Q g H$ and crossed subprocesses with vector boson exchange in the $t$-channel. We explicitly exclude $s$-channel weak boson exchange and thus set aside higgsstrahlung processes, i.e. $V H j$ production with subsequent decay
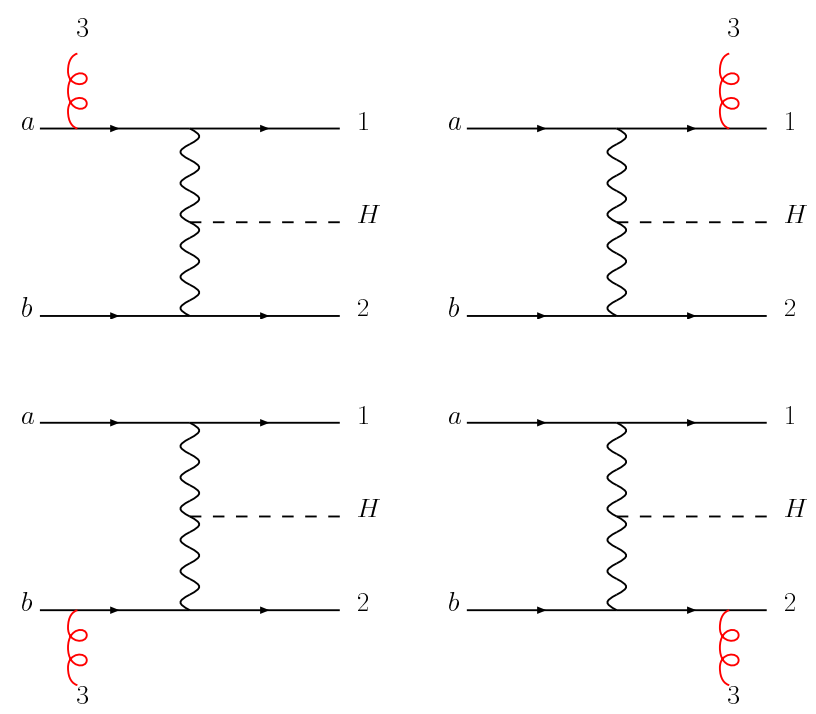

Figure 1: Lowest-order Feynman graphs for $p p \rightarrow H j j j$ via $\mathrm{VBF}$. $V \rightarrow j j$. In the following, higgsstrahlung

is viewed as a separate process and we also neglect any interference of VBF and higgsstrahlung (in the case of identical fermion flavors) since these interference effects are very small in the phase space region relevant for VBF observation at the LHC [21, 22].

In order to clarify our notation and the approximations in our calculation, let us start by considering the form of the Born amplitude for the $q Q \rightarrow q Q g H$ subprocess. By $\mathcal{M}_{3}\left(1_{q}, 2_{Q}, 3_{g}, a_{q}, b_{Q}\right)$ we denote the matrix element for the parton level process

$$
q\left(p_{a}\right)+Q\left(p_{b}\right) \rightarrow q\left(p_{1}\right)+Q\left(p_{2}\right)+g\left(p_{3}\right)+H(P),
$$

shown in Fig. 1. Two distinct color structures contribute to this Born matrix element with three final state colored partons,

$$
\begin{aligned}
\mathcal{M}_{3}\left(1_{q}, 2_{Q}, 3_{g}, a_{q}, b_{Q}\right) & =\mathcal{A}_{3}\left(1_{q}, 3_{g}, a_{q} ; 2_{Q}, b_{Q}\right) \delta_{i_{2} i_{b}} t_{i_{1} i_{a}}^{a_{3}} \\
& +\mathcal{A}_{3}\left(2_{Q}, 3_{g}, b_{Q} ; 1_{q}, a_{q}\right) \delta_{i_{1} i_{a}} t_{i_{2} i_{b}}^{a_{3}} .
\end{aligned}
$$

Focusing on the gluon emission, each of the amplitudes $\mathcal{A}_{3}$ can be viewed as a Compton scattering amplitude for the process $Q\left(k_{1}\right) \rightarrow Q\left(k_{2}\right) g\left(q_{1}\right) V\left(q_{2}\right)$, defined by

$$
\begin{aligned}
\mathcal{M}_{B}\left(k_{2}, q_{1}, q_{2} ; \epsilon_{1}, \epsilon_{2}\right) & =-e g_{\tau}^{V Q_{2} Q_{1}} g_{s} \bar{\psi}\left(k_{2}\right)\left\{\gamma^{\nu} \frac{\left(\not k_{2}+q_{2}\right)}{\left(k_{2}+q_{2}\right)^{2}} \gamma^{\mu}\right. \\
& \left.+\gamma^{\mu} \frac{\left(\not k_{2}+q_{1}\right)}{\left(k_{2}+q_{1}\right)^{2}} \gamma^{\nu}\right\} P_{\tau} \psi\left(k_{1}\right) \epsilon_{1 \mu}\left(q_{1}\right) \epsilon_{2 \nu}\left(q_{2}\right) .
\end{aligned}
$$

Here, $-e g_{\tau}^{V Q_{2} Q_{1}}$ is the left- or righthanded coupling of the quarks to the weak boson, $g_{s}$ denotes the strong coupling constant, $P_{\tau}=\frac{1}{2}\left(1+\tau \gamma^{5}\right)$ is the chirality projector, and $\epsilon_{1}$ and $\epsilon_{2}$ are the polarization vectors of the gluon and of the weak boson, respectively. The 
role of the polarization vector for the weak boson is taken by a current, $h^{\mu}$, which, for the first two diagrams in Fig. 1, is given by

$$
h^{\mu}\left(p_{b} \tau_{b}, p_{2} \tau_{2}\right)=\delta_{\tau_{2} \tau_{b}}(-e) g_{H V V} g_{\tau_{2}}^{V f_{2} f_{b}} D_{V}\left[p_{a 13}^{2}\right] D_{V}\left[p_{b 2}^{2}\right] \bar{\psi}\left(p_{2}\right) \gamma^{\mu} P_{\tau_{2}} \psi\left(p_{b}\right)
$$

with $p_{i j k}=p_{i}-p_{j}-p_{k}$ and $p_{i j}=p_{i}-p_{j}$, while $D_{V}\left[q^{2}\right]=1 /\left[q^{2}-M_{V}^{2}\right]$ is the weak boson propagator, which, in our calculation, only occurs with space-like momentum. In terms of the Compton amplitude of Eq. (2.3) the $\mathcal{A}_{3}$ are then given by

$$
\begin{aligned}
& \mathcal{A}_{3}\left(1_{q}, 3_{g}, a_{q} ; 2_{Q}, b_{Q}\right)=\mathcal{M}_{B}\left(p_{1}, p_{3}, p_{a 13} ; \epsilon_{3}, h\left(p_{b} \tau_{b}, p_{2} \tau_{2}\right)\right) \\
& \mathcal{A}_{3}\left(2_{Q}, 3_{g}, b_{Q} ; 1_{q}, a_{q}\right)=\mathcal{M}_{B}\left(p_{2}, p_{3}, p_{b 23} ; \epsilon_{3}, h\left(p_{a} \tau_{a}, p_{1} \tau_{1}\right)\right) .
\end{aligned}
$$

The $g Q \rightarrow q \bar{q} Q H$ subprocess is obtained by crossing the initial state quark $q\left(p_{a}\right)$ with the final state gluon in Eq. (2.2) and dropping the $s$-channel graphs which result from crossing the diagrams in the second line of Fig. 1. The 3-parton matrix elements $\mathcal{M}_{3}$ have been computed using the helicity amplitude method of Ref. 23].

The real emission corrections to VBF $H j j j$ production consist of four subprocess classes with four final state partons. These classes are (a) $q Q \rightarrow q Q g g H$, (b) $q Q \rightarrow$ $q Q q^{\prime} \bar{q}^{\prime} H$, (c) $g Q \rightarrow q \bar{q} Q g H$, and (d) $g g \rightarrow q \bar{q} Q \bar{Q} H$. The generalization to the crossed processes with $q \rightarrow \bar{q}$ and/or $Q \rightarrow \bar{Q}$ is straightforward.

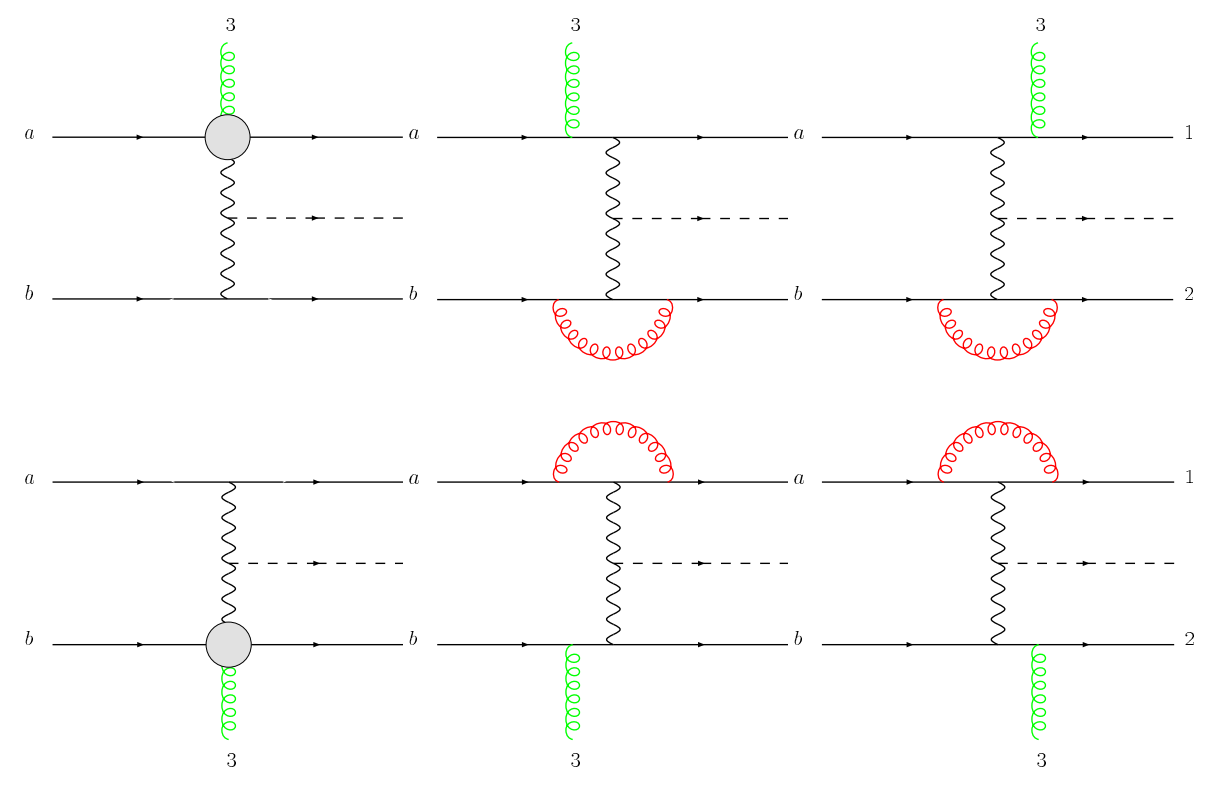

Figure 2: The dominant virtual QCD corrections. The "blobs" correspond to the sum of all virtual corrections to the basic $Q \rightarrow Q g V$ Compton amplitude and are given more explicitly in Fig. 6. The first diagram and the second pair of diagrams in each line form gauge invariant subsets.

The above subprocesses lead to soft and collinear singularities when integrated over the phase space of the final state partons. We use the Catani-Seymour dipole subtraction method to regulate these divergences [26] and to cancel them against those originating from the virtual corrections. The virtual corrections can be divided into two classes of gauge 
covariant subsets. The first class (depicted in Fig. 2) are graphs in which the internal gluon propagator is attached to a single fermion line and which involve up to box corrections. The second class (depicted in Fig. 3) are graphs in which the internal gluon propagates between different fermion lines, i.e. they contain a $t$-channel gluon. These graphs only play a role for subprocesses with two initial state quarks or anti-quarks. For gluon initiated processes they only contribute to the interference of VBF and higgsstrahlung diagrams, which we neglect. The interference of the hexagon and pentagon amplitudes with the Born amplitude is color suppressed by a factor $d_{G}=N^{2}-1$ with respect to the interference of box corrections with the Born amplitude. We neglect the contribution of the hexagon and pentagon amplitudes. However, in doing so we must also consider the color structure of the real corrections and drop contributions which cancel the infrared singularities of the pentagons and hexagons.

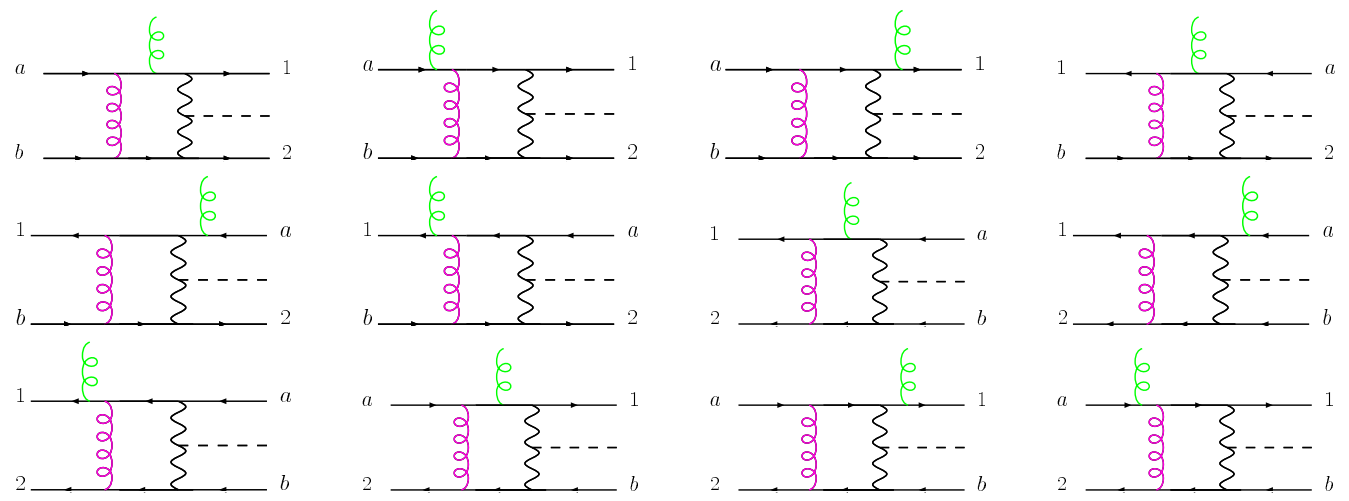

Figure 3: Pentagon and hexagon diagrams for the color structure $\delta_{i_{1} i_{a}} t_{i_{2} i_{b}}^{a_{3}}$. An analogous set appears with the external gluon attached to the lower quark line. Note that hexagon graphs with a three-gluon-vertex correspond to a color structure which cannot interfere with the Born amplitude.

As an example for these real emission processes, consider the matrix element for the subprocess,

$$
q\left(p_{a}\right)+Q\left(p_{b}\right) \rightarrow q\left(p_{1}\right)+Q\left(p_{2}\right)+g\left(p_{3}\right)+g\left(p_{4}\right)+H(P)
$$

depicted in Fig. 4 and denoted by $\mathcal{M}_{4}\left(1_{q}, 2_{Q}, 3_{g}, 4_{g}, a_{q}, b_{Q}\right)$. $\mathcal{M}_{4}$ has the following color decomposition in terms of color subamplitudes, $\mathcal{A}$ and $\mathcal{B}$,

$$
\begin{aligned}
\mathcal{M}_{4}\left(1_{q}, 2_{Q}, 3_{g}, 4_{g}, a_{q}, b_{Q}\right) & =\left(t^{a_{3}} t^{a_{4}}\right)_{i_{1} i_{a}} \delta_{i_{2} i_{b}} \mathcal{A}\left(1_{q}, 3_{g}, 4_{g}, a_{q} ; 2_{Q}, b_{Q}\right) \\
& +\left(t^{a_{4}} t^{a_{3}}\right)_{i_{1} i_{a}} \delta_{i_{2} i_{b}} \mathcal{A}\left(1_{q}, 4_{g}, 3_{g}, a_{q} ; 2_{Q}, b_{Q}\right) \\
& +\left(t^{a_{3}} t^{a_{4}}\right)_{i_{2} i_{b}} \delta_{i_{1} i_{a}} \mathcal{A}\left(2_{Q}, 3_{g}, 4_{g}, b_{Q} ; 1_{q}, a_{q}\right) \\
& +\left(t^{a_{4}} t^{a_{3}}\right)_{i_{2} i_{b}} \delta_{i_{1} i_{a}} \mathcal{A}\left(2_{Q}, 4_{g}, 3_{g}, b_{Q} ; 1_{q}, a_{q}\right) \\
& +t_{i_{1} i_{a}}^{a_{3}} t_{i_{2} i_{b}}^{a_{4}} \mathcal{B}\left(1_{q}, 3_{g}, a_{q} ; 2_{Q}, 4_{g}, b_{Q}\right) \\
& +t_{i_{1} i_{a}}^{a_{4}} t_{i_{2} i_{b}}^{a_{3}} \mathcal{B}\left(1_{q}, 4_{g}, a_{q} ; 2_{Q}, 3_{g}, b_{Q}\right) .
\end{aligned}
$$

The $\mathcal{A}$ terms correspond to both gluons attached to the same quark line, while $\mathcal{B}$ terms describe emission of one gluon from each of the two quark lines. Abbreviating these amplitudes by

$$
\mathcal{A}_{1}=\mathcal{A}\left(1_{q}, 3_{g}, 4_{g}, a_{q} ; 2_{Q}, b_{Q}\right), \quad \mathcal{A}_{2}=\mathcal{A}\left(1_{q}, 4_{g}, 3_{g}, a_{q} ; 2_{Q}, b_{Q}\right),
$$




$$
\begin{array}{ll}
\mathcal{A}_{3}=\mathcal{A}\left(2_{Q}, 3_{g}, 4_{g}, b_{Q} ; 1_{q}, a_{q}\right), & \mathcal{A}_{4}=\mathcal{A}\left(2_{Q}, 4_{g}, 3_{g}, b_{Q} ; 1_{q}, a_{q}\right), \\
\mathcal{B}_{1}=\mathcal{B}\left(1_{q}, 3_{g}, a_{q} ; 2_{Q}, 4_{g}, b_{Q}\right), & \mathcal{B}_{2}=\mathcal{B}\left(1_{q}, 4_{g}, a_{q} ; 2_{Q}, 3_{g}, b_{Q}\right),
\end{array}
$$

the color summed squared matrix element can be written as

$$
\begin{aligned}
& \left|\mathcal{M}_{4}\left(1_{q}, 2_{Q}, 3_{g}, 4_{g}, a_{q}, b_{Q}\right)\right|^{2}=d_{F}^{2} C_{F}^{2}\left\{\left|\mathcal{A}_{1}\right|^{2}+\left|\mathcal{A}_{2}\right|^{2}+\left|\mathcal{A}_{3}\right|^{2}+\left|\mathcal{A}_{4}\right|^{2}+\left|\mathcal{B}_{1}\right|^{2}+\left|\mathcal{B}_{2}\right|^{2}\right. \\
& \left.\quad+2 x \operatorname{Re}\left[\mathcal{A}_{1} \mathcal{A}_{2}^{*}+\mathcal{A}_{3} \mathcal{A}_{4}^{*}\right]+2 y \operatorname{Re}\left[\left(\mathcal{A}_{1}+\mathcal{A}_{2}\right) \cdot\left(\mathcal{A}_{3}+\mathcal{A}_{4}\right)^{*}+\mathcal{B}_{1} \mathcal{B}_{2}^{*}\right]\right\}
\end{aligned}
$$

with $x=1-C_{A} / 2 C_{F}=-1 /\left(N^{2}-1\right)$ and $y=1 / d_{G}=1 /\left(N^{2}-1\right)$, where the explicit value is given for an $S U(N)$ gauge group, with $d_{G}=N^{2}-1$ and $d_{F}=N$. The term in Eq. (2.9) which is proportional to $y$ leads to a soft divergence when integrated over the phase space of the soft/collinear parton, which is in fact canceled by the corresponding soft divergent hexagon and pentagon graphs. Since we neglect the latter, for consistency, we also need to set $y=0$ in Eq. (2.9). The association of the $y$-terms with the hexagon and pentagon diagrams of Fig. 3 is made clear when recognizing that these are all the contributions where the same gluon is attached to both an upper and a lower quark line. The $y$-term and the interference of hexagons and pentagons with the Born amplitude are not only color suppressed by a factor $1 /\left(N^{2}-1\right)$, they are further suppressed because the interfering amplitudes are never large simultaneously when typical VBF cuts are applied. Consider, for example, the $\mathcal{B}_{1}$ and $\mathcal{B}_{2}$ amplitudes in Fig. 1 . $\mathcal{B}_{1}$ is large when $q_{1}$ and $g_{3}$ are forward (i.e. in the initial $q_{a}$ direction) and $q_{2}$ and $g_{4}$ are backward, in the $q_{b}$ hemisphere. For $\mathcal{B}_{2}$ to be large, $q_{1}$ and $g_{4}$ must be forward while $q_{2}$ and $g_{3}$ are backwards. These conditions cannot be satisfied simultaneously for a large rapidity separation between the highest $p_{T}$ jets, which typically will be the two quark jets. The largest interference between $\mathcal{B}_{1}$ and $\mathcal{B}_{2}$ and, similarly, between $\mathcal{A}_{1}+\mathcal{A}_{2}$ and $\mathcal{A}_{3}+\mathcal{A}_{4}$ is to be expected when both factors in the interference terms have similar size, i.e. when both gluons are emitted in the central region. For central gluons, however, all contributing amplitudes are suppressed due to the gluon radiation pattern of the underlying $t$-channel weak boson exchange.

We have estimated the error on the total $H j j j$ cross section, $\Delta \sigma^{N L O}$, which we make in neglecting the hexagon and pentagon topologies (shown in Fig. 3) and the corresponding interference terms ( $y$-terms) in Eq. (2.9). Consider the dominant phase space region where one gluon (say $\left.g_{3}\right)$ is hard and the second one $\left(g_{4}\right)$ is soft. The soft emission can be factorized as an eikonal factor, while the hard part of $\mathcal{B}_{1}$ and $\mathcal{A}_{1}+\mathcal{A}_{2}$ will be given by the upper line of the Born diagram of Fig. 目, i.e. by $\mathcal{A}_{3}\left(1_{q}, 3_{g}, a_{q} ; 2_{Q}, b_{Q}\right)$. Analogously, the hard factor in $\mathcal{B}_{2}$ and $\mathcal{A}_{3}+\mathcal{A}_{4}$ is $\mathcal{A}_{3}\left(2_{Q}, 3_{g}, b_{Q} ; 1_{q}, a_{q}\right)$, corresponding to hard emission from the lower quark line in Fig. 1. Approximately, in the soft region, the $y$-terms plus the corresponding virtual corrections, given by the interference of hexagons and pentagons with the Born amplitude, are proportional to the product

$$
\frac{\alpha_{s}}{2 \pi} \frac{d_{F}^{2} C_{F}^{2}}{\left(N^{2}-1\right)} 2 \operatorname{Re}\left[\mathcal{A}_{3}\left(1_{q}, 3_{g}, a_{q} ; 2_{Q}, b_{Q}\right) \mathcal{A}_{3}\left(2_{Q}, 3_{g}, b_{Q} ; 1_{q}, a_{q}\right)^{*}\right],
$$

integrated over the 3-parton phase space. In Fig. 5, we compare the absolute value of this proxy for the full interference terms (dotted blue curves) with the tree level cross section (dashed red curve). Shown is the distribution in rapidity for the veto jet (lowest $p_{T}$ parton) 

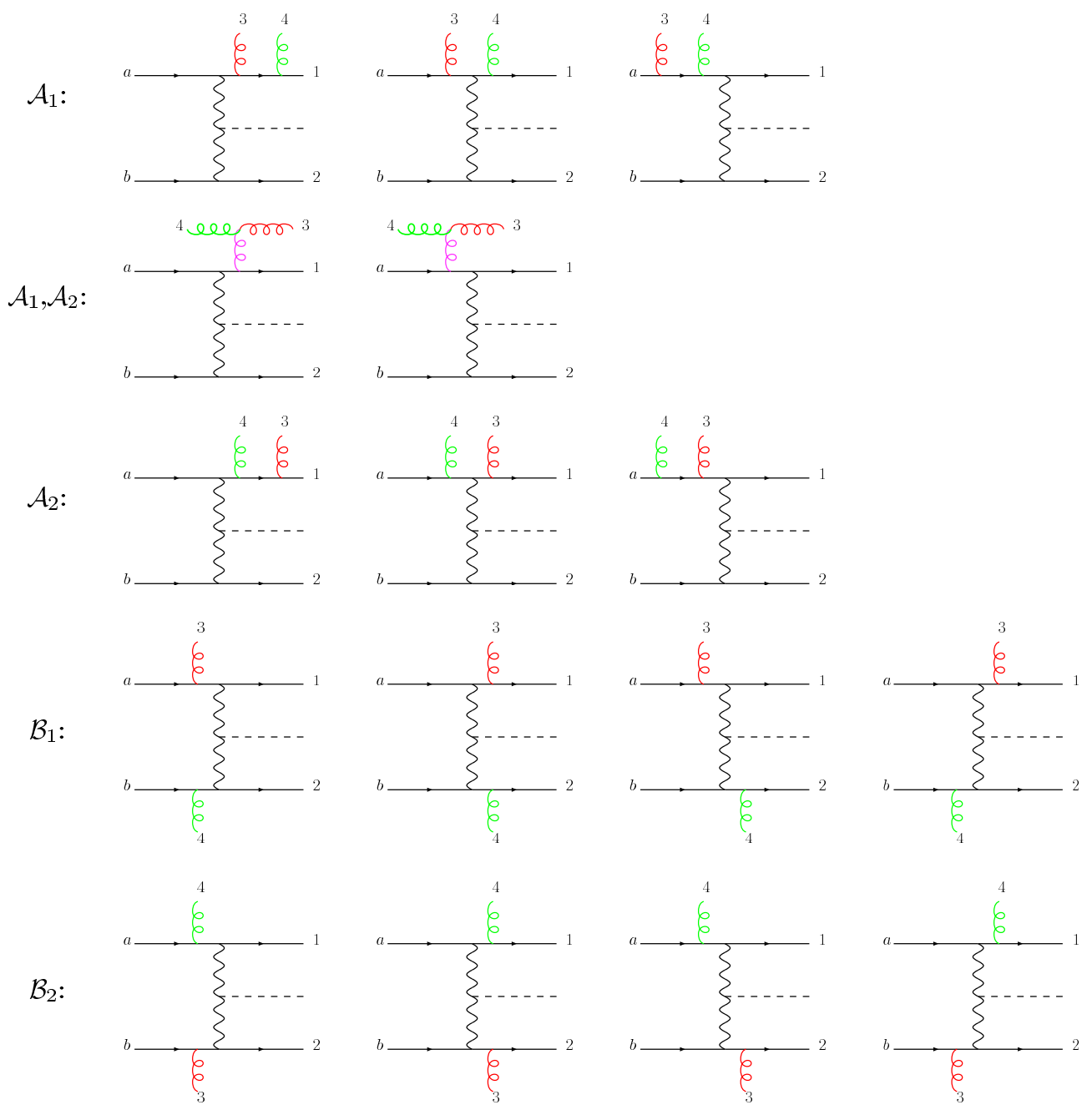

Figure 4: Feynman graphs for the real emission amplitude $\mathcal{M}_{4}\left(1_{q}, 2_{Q}, 3_{g}, 4_{g}, a_{q}, b_{Q}\right)$ as described in Eq. (2.7)

measured with respect to the center of the rapidity of the two tagging jets. In the right panel of Fig. 5, the ratio of the two distributions is shown. As a second estimator for the neglected terms, we have calculated the full $y$-terms for the 4-parton final state and soft approximations for the hexagons and pentagons, by keeping the infrared divergent $C$-function terms only, according to the prescription of Ref. [24]. For both contributions the full Catani-Seymour subtraction has been implemented, with dipole terms as listed for $y=1 / d_{G}$ in Table 1 . The resulting curve for

$$
R\left(y_{\mathrm{rel}}\right)=\frac{d \Delta \sigma^{N L O}\left(\mu_{R}, \mu_{F}\right) / d y_{\mathrm{rel}}}{d \sigma^{L O}\left(\mu_{R}, \mu_{F}\right) / d y_{\mathrm{rel}}}
$$

is shown for $\mu_{R}=\mu_{F}=40 \mathrm{GeV}$. The ratio, $R$, reaches a maximal value of $\approx 10^{-4}$ in the central region between the two tagging jets, in agreement with the result for the proxy discussed above. We conclude that the $y$-terms and the corresponding hexagon and pentagon contributions give a relative contribution below one permille everywhere in phase 
space and can safely be neglected. We note that these interference terms are at the same level as the interference between gluon fusion and vector boson fusion for $p p \rightarrow H j j j$. In a complete calculation, not only would the hexagon and pentagon graphs need to be calculated, gluon fusion contributions would have to be included as well.
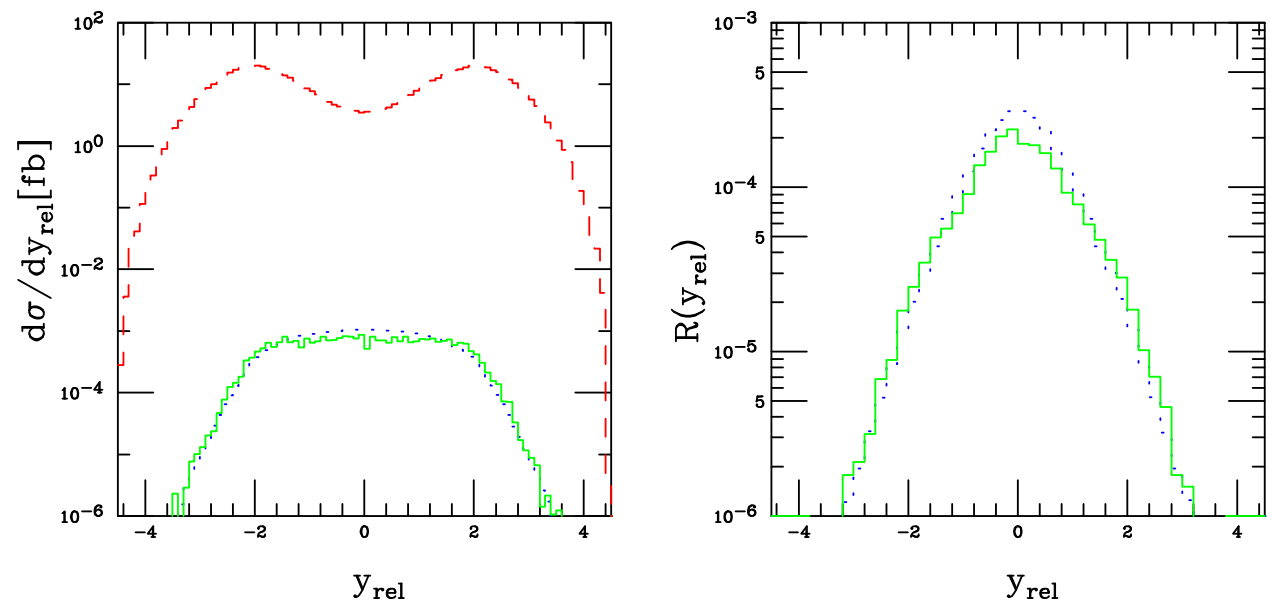

Figure 5: The distribution in rapidity of the veto jet measured with respect to the center of rapidity of the tagging jets. In the left panel, $d \sigma / d y_{\text {rel }}$ is shown at LO (dashed red), for the proxy given by Eq. (2.10) (dotted blue), and for the NLO color suppressed contribution in the soft approximation (solid green). The right panel depicts the ratio, $R$, for both the proxy (dotted blue) and the NLO color suppressed contribution (solid green).

In addition to the $y$-terms discussed above we also neglect any interference terms for identical fermions in our simulations. These terms are color suppressed by a factor $1 / N$ and can only contribute when fermion helicities are the same. For charged current contributions we have determined the size of these interference terms for 4-quark final states and have compared them to the charged current contribution to the LO 3-jet cross section. We find a relative contribution of $7.5 \cdot 10^{-4}$ within the cuts of Section 3: also these "Pauli interference terms" are truly negligible. With these approximations, the fortran code for the real emission matrix element squared was generated with the help of MadGraph [25].

The 4-parton phase space integral of the squared real matrix elements suffers from soft and collinear divergences. The dipole subtraction method of Catani and Seymour provides a means to regulate these divergences [26]. In the Catani-Seymour formalism the NLO corrections consist of three pieces: (a) the contribution of the dipole subtracted real corrections, $\sigma_{4}^{N L O}$, (b) the contribution of the finite virtual corrections, $\sigma_{3}^{N L O}$, and (c) a piece resulting from the factorization of collinear singularities into the parton distribution functions, $\sigma_{3, \mathrm{col}}^{N L O}$. As an example, consider the process, $q Q \rightarrow q Q g g H$, in the $y=0$ case in Eq. (2.9). The subtracted cross section for this process takes the form,

$$
\begin{aligned}
\sigma_{4}^{N L O}(q Q \rightarrow q Q g g H) & =\int_{0}^{1} d x_{a} \int_{0}^{1} d x_{b} f_{q / p}\left(x_{a}, \mu_{F}\right) f_{Q / p}\left(x_{b}, \mu_{F}\right) \frac{1}{2 \hat{s}} d \Phi_{5}\left(p_{a}, p_{b}\right) \\
& \cdot\left\{\left|\mathcal{M}_{4}\left(1_{q}, 2_{Q}, 3_{g}, 4_{g}, a_{q}, b_{Q}\right)\right|^{2} F_{J}^{(4)}\left(p_{1}, p_{2}, p_{3}, p_{4} ; p_{a}+p_{b}\right)\right.
\end{aligned}
$$




\begin{tabular}{|c|c|}
\hline real subprocess & dipole factors \\
\hline$\left(1_{q}, 2_{Q}, 3_{g}, 4_{g}, a_{q}, b_{Q}\right)$ & $\mathcal{D}_{14,3}, \mathcal{D}_{13,4}, \mathcal{D}_{34,1}, \mathcal{D}_{14}^{a}, \mathcal{D}_{13}^{a}$ \\
$y=0$ in Eq. (2.9) & $\mathcal{D}_{34}^{a}, \mathcal{D}_{1}^{a 4}, \mathcal{D}_{1}^{a 3}, \mathcal{D}_{3}^{a 4}, \mathcal{D}_{4}^{a 3}$ \\
& $\mathcal{D}_{24,3}, \mathcal{D}_{23,4}, \mathcal{D}_{34,2}, \mathcal{D}_{24}^{b}, \mathcal{D}_{23}^{b}$ \\
& $\mathcal{D}_{34}^{b}, \mathcal{D}_{2}^{b 4}, \mathcal{D}_{2}^{b 3}, \mathcal{D}_{3}^{b 4}, \mathcal{D}_{4}^{b 3}$ \\
\hline$y=1 / d_{G}$ in Eq. (2.9) & $\mathcal{D}^{a 3, b}, \mathcal{D}^{b 3, a}, \mathcal{D}^{a 4, b}, \mathcal{D}^{b 4, a}, \mathcal{D}_{24}^{a}$ \\
& $\mathcal{D}_{14}^{b}, \mathcal{D}_{2}^{a 4}, \mathcal{D}_{1}^{b 4}, \mathcal{D}_{24,1}, \mathcal{D}_{14,2}$ \\
& $\mathcal{D}_{23}^{a}, \mathcal{D}_{13}^{b}, \mathcal{D}_{2}^{a 3}, \mathcal{D}_{1}^{b 3}, \mathcal{D}_{23,1}, \mathcal{D}_{13,2}$ \\
\hline$\left(1_{q}, 2_{Q}, 3_{q^{\prime}}, 4_{\bar{q}^{\prime}}, a_{q}, b_{Q}\right)$ & $\mathcal{D}_{34,1}, \mathcal{D}_{31,2}, \mathcal{D}_{34}^{a}, \mathcal{D}_{34}^{b}$ \\
& $\mathcal{D}_{3}^{a 1}, \mathcal{D}_{4}^{a 1}, \mathcal{D}_{3}^{b 2}, \mathcal{D}_{4}^{b 2}$ \\
\hline$\left(1_{q}, 2_{Q}, 3_{\bar{q}}, 4_{g}, a_{g}, b_{Q}\right)$ & $\mathcal{D}_{1}^{a 3}, \mathcal{D}_{3}^{a 1}, \mathcal{D}_{4}^{a 3}, \mathcal{D}_{4}^{a 1}$ \\
& $\mathcal{D}_{1}^{a 4}, \mathcal{D}_{3}^{a 4}, \mathcal{D}_{2}^{b 4}, \mathcal{D}_{24}^{b}$ \\
& $\mathcal{D}_{14}^{a}, \mathcal{D}_{34}^{a}, \mathcal{D}_{14,3}, \mathcal{D}_{34,1}$ \\
\hline$\left(1_{q}, 2_{Q}, 3_{\bar{q}}, 4_{\bar{Q}}, a_{g}, b_{g}\right)$ & $\mathcal{D}_{2}^{b 4}, \mathcal{D}_{4}^{b 2}, \mathcal{D}_{1}^{a 3}, \mathcal{D}_{3}^{a 1}$ \\
\hline
\end{tabular}

Table 1: Dipole factors for the real emission corrections to $H j j j$ production. The $y=1 / d_{G}$ line gives the additional dipole factors which are needed for the $q Q \rightarrow q Q g g H$ process when the $y$ terms in Eq. (2.9) are not neglected.

$$
\begin{aligned}
& -\sum_{\substack{\text { pairs } \\
i, j}} \sum_{k \neq i, j} \mathcal{D}_{i j, k}\left(p_{1}, p_{2}, p_{3}, p_{4} ; p_{a}, p_{b}\right) F_{J}^{(3)}\left(p_{1}, . . \tilde{p}_{i j}, \tilde{p}_{k}, . . p_{4} ; p_{a}, p_{b}\right) \\
& -\sum_{\substack{\text { pairs } \\
i, j}}\left[\mathcal{D}_{i j}^{a}\left(p_{1}, p_{2}, p_{3}, p_{4}, ; p_{a}, p_{b}\right) F_{J}^{(3)}\left(p_{1}, . . \tilde{p}_{i j}, . ., p_{4} ; \tilde{p}_{a}, p_{b}\right)+(a \leftrightarrow b)\right] \\
& \left.-\sum_{i} \sum_{k \neq i}\left[\mathcal{D}_{k}^{a i}\left(p_{1}, p_{2}, p_{3}, p_{3} ; p_{a}, p_{b}\right) F_{J}^{(3)}\left(p_{1}, . . \tilde{p}_{k}, \ldots, p_{4} ; \tilde{p}_{a i}, p_{b}\right)+(a \leftrightarrow b)\right]\right\},
\end{aligned}
$$

where the $\mathcal{D}_{i j, k}$ etc. are the dipole factors as defined in Ref. [26], $d \Phi_{5}$ is the 5 -particle phase space measure and $\hat{s}=\left(p_{a}+p_{b}\right)^{2}$ denotes the center-of-mass energy. A complete list of the dipole factors in Eq. (2.12) is shown in Table 1. Notice, that we do not need to consider dipole factors for which there is an initial state singularity with an initial state spectator for the case of $y=0$ because in this approximation radiative corrections to the upper and the lower lines in Fig. 1 effectively decouple. We also show in Table 1 dipole factors for quark-gluon and gluon-gluon initiated processes. The functions $F_{J}^{(3)}$ and $F_{J}^{(4)}$ define the jet algorithm for 4-parton and 3-parton final states and must be infrared safe which formally means that $F_{J}^{(4)} \rightarrow F_{J}^{(3)}$ in any case where the 4-parton and 3-parton configurations are kinematically degenerate.

The dipole factors are integrated in $d=4-2 \epsilon$ space-time dimension over the phase space of the soft/collinear parton. Integrating the dipole factors for the processes, $q Q \rightarrow$ $q Q g g H$ and $q Q \rightarrow q Q q^{\prime} \bar{q}^{\prime} H$, lead to the universal singular factor, $<\mathbf{I}(\epsilon)>$. For the parton level process

$$
q\left(p_{a}\right)+Q\left(p_{b}\right) \rightarrow q\left(p_{1}\right)+Q\left(p_{2}\right)+g\left(p_{3}\right)+H(P),
$$


we can split $<\mathbf{I}(\epsilon)>$ into two pieces according to,

$$
<\mathbf{I}(\epsilon)>=C_{F}\left(\mathcal{I}_{1}(\epsilon)+\mathcal{I}_{2}(\epsilon)\right) .
$$

$\mathcal{I}_{1}(\epsilon)$ is a piece proportional to the Born-level color subamplitude squared, $\left|\mathcal{A}_{3}\left(1_{q}, 3_{g}, a_{q} ; 2_{Q}, b_{Q}\right)\right|^{2}$, and is

$$
\begin{aligned}
\mathcal{I}_{1}(\epsilon)= & \left|\mathcal{A}_{3}\left(1_{q}, 3_{g}, a_{q} ; 2_{Q}, b_{Q}\right)\right|^{2} \frac{\alpha_{s}\left(\mu_{R}^{2}\right)}{2 \pi} \frac{1}{\Gamma(1-\epsilon)} \\
& \cdot\left\{\frac{1}{2}\left(\left(\frac{4 \pi \mu_{R}^{2}}{s_{13}}\right)^{\epsilon}+\left(\frac{4 \pi \mu_{R}^{2}}{s_{a 3}}\right)^{\epsilon}\right)\left(\frac{C_{A}}{\epsilon^{2}}+\frac{\gamma_{g}}{\epsilon}+\gamma_{g}+K_{g}\right)\right. \\
+ & \frac{1}{2} \frac{C_{A}}{C_{F}}\left(\left(\frac{4 \pi \mu_{R}^{2}}{s_{13}}\right)^{\epsilon}+\left(\frac{4 \pi \mu_{R}^{2}}{s_{a 3}}\right)^{\epsilon}-2\left(\frac{4 \pi \mu_{R}^{2}}{s_{a 1}}\right)^{\epsilon}\right)\left(\frac{C_{F}}{\epsilon^{2}}+\frac{\gamma_{q}}{\epsilon}+\gamma_{q}+K_{q}\right) \\
+ & \left.2\left(\left(\frac{4 \pi \mu_{R}^{2}}{s_{a 1}}\right)^{\epsilon}+\left(\frac{4 \pi \mu_{R}^{2}}{s_{b 2}}\right)^{\epsilon}\right)\left(\frac{C_{F}}{\epsilon^{2}}+\frac{\gamma_{q}}{\epsilon}+\gamma_{q}+K_{q}\right)\right\}
\end{aligned}
$$

with

$$
\gamma_{q}=\frac{3}{2} C_{F}, \quad \gamma_{g}=\frac{11}{6} C_{A}-\frac{2}{3} T_{R} N_{f}
$$

and,

$$
K_{q}=\left(\frac{7}{2}-\frac{\pi^{2}}{6}\right) C_{F}, \quad K_{g}=\left(\frac{67}{18}-\frac{\pi^{2}}{6}\right) C_{A}-\frac{10}{9} T_{R} N_{f}
$$

Here $s_{i j}=2 p_{i} \cdot p_{j}$ with $i=1,2,3, a$ or $b . T_{R}=1 / 2, C_{A}=N$, and $C_{F}=\left(N^{2}-1\right) /(2 N)$ in $S U(N)$ gauge theory. The number of flavors is $N_{f}=5 . \mathcal{I}_{2}(\epsilon)$ is obtained from $\mathcal{I}_{1}(\epsilon)$ by interchanging the quark labels, $a \leftrightarrow b$ and $1 \leftrightarrow 2$. The $1 / \epsilon^{2}$ and $1 / \epsilon$ divergences cancel against the virtual corrections shown in Fig. 2.

In our approximation there are two distinct color structures that contribute to this virtual matrix element, $\mathcal{M}_{3}^{\text {virt }}\left(1_{q}, 2_{Q}, 3_{g}, a_{q}, b_{Q}\right)$, with three final state colored partons,

$$
\begin{aligned}
\mathcal{M}_{3}^{\text {virt }}\left(1_{q}, 2_{Q}, 3_{g}, a_{q}, b_{Q}\right) & =\mathcal{A}_{3}^{\text {virt }}\left(1_{q}, 3_{g}, a_{q} ; 2_{Q}, b_{Q}\right) \delta_{i_{2} i_{b}} t_{i_{1} i_{a}}^{a_{3}} \\
& +\mathcal{A}_{3}^{\text {virt }}\left(2_{Q}, 3_{g}, b_{Q} ; 1_{q}, a_{q}\right) \delta_{i_{1} i_{a}} t_{i_{2} i_{b}}^{a_{3}} .
\end{aligned}
$$

The interference between the virtual and Born three parton amplitudes takes on the following form upon summing over final state colors and averaging over initial state colors,

$$
\begin{aligned}
& \overline{\sum_{\text {colors }}} 2 \operatorname{Re}\left[\mathcal{M}_{3}^{\text {virt }} \mathcal{M}_{3}^{*}\right]=C_{F}\left(2 \operatorname{Re}\left[\mathcal{A}_{3}^{\text {virt }}\left(1_{q}, 3_{g}, a_{q} ; 2_{Q}, b_{Q}\right) \mathcal{A}_{3}^{*}\left(1_{q}, 3_{g}, a_{q} ; 2_{Q}, b_{Q}\right)\right]\right. \\
& \left.+\quad 2 \operatorname{Re}\left[\mathcal{A}_{3}^{\mathrm{virt}}\left(2_{Q}, 3_{g}, b_{Q} ; 1_{q}, a_{q}\right) \mathcal{A}_{3}^{*}\left(2_{Q}, 3_{g}, b_{Q} ; 1_{q}, a_{q}\right)\right]\right) .
\end{aligned}
$$

We split the virtual corrections shown in Fig. 2 into two classes: the virtual corrections along a quark line with only one weak boson attached and the virtual corrections along a quark line with a gluon and a weak boson attached. The former, with only a weak boson vertex, are factorizable in terms of the tree-level current $h^{\mu}$ defined by Eq. (2.4). For vertex corrections to the lower line one has

$$
h_{\mathrm{virt}}^{\mu}\left(p_{b} \tau_{b}, p_{2} \tau_{2}\right)=h^{\mu}\left(p_{b} \tau_{b}, p_{2} \tau_{2}\right) C_{F} \frac{\alpha_{s}\left(\mu_{R}\right)}{4 \pi}\left(\frac{4 \pi \mu_{R}^{2}}{s_{b 2}}\right)^{\epsilon} \frac{1}{\Gamma(1-\epsilon)}\left(-\frac{2}{\epsilon^{2}}-\frac{3}{\epsilon}-8\right),
$$



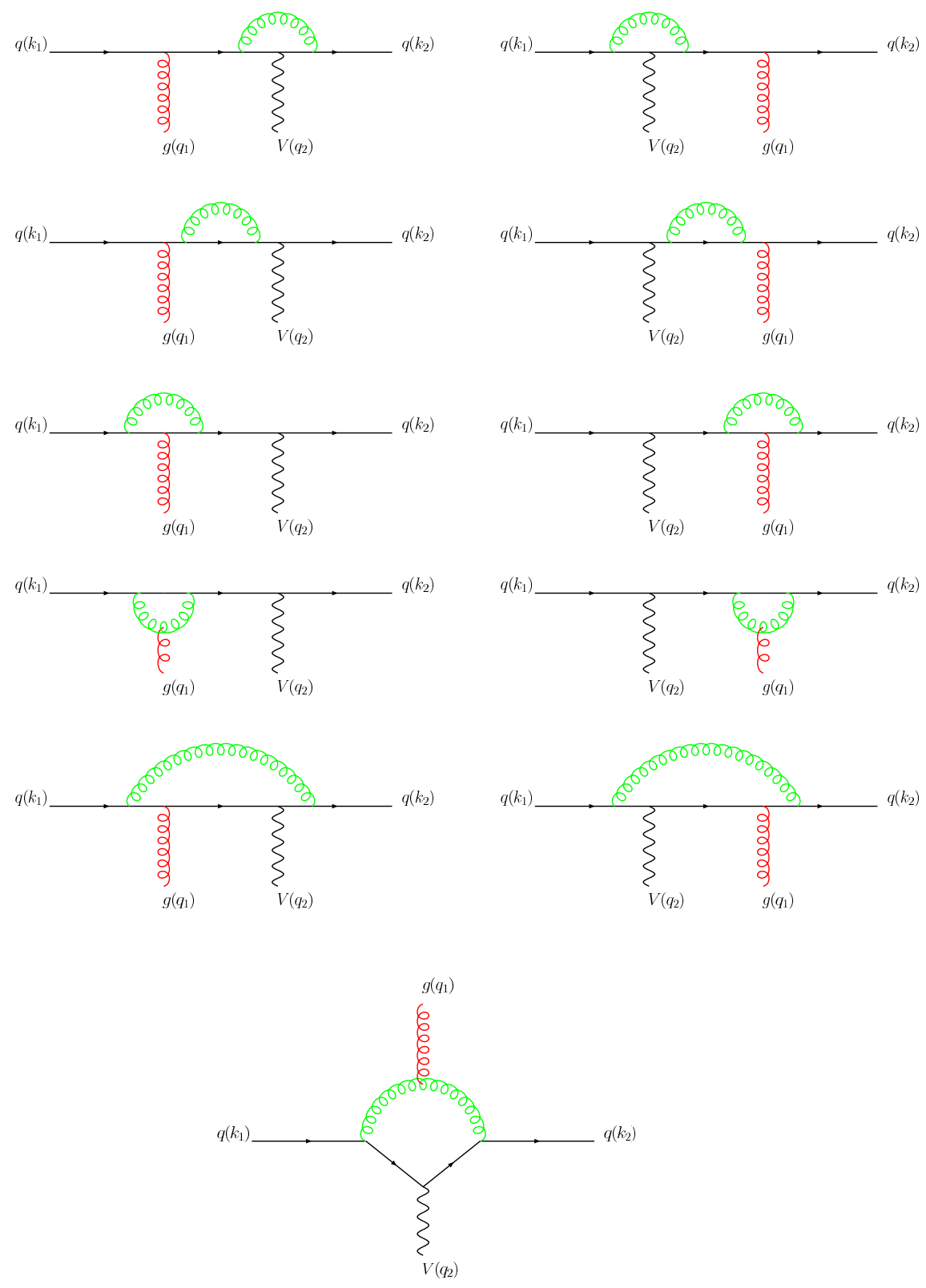

Figure 6: The one-loop QCD corrections for $q \rightarrow q g V$.

and similarly for vertex corrections to the upper line. Here $\mu_{R}$ is the renormalization scale, and $s_{b 2}=2 p_{b} \cdot p_{2}$ is the weak boson virtuality for massless quarks.

The second class of diagrams, corresponding to the "blob" in Fig. 2 and shown explicitly in Fig. 6, are the virtual QCD corrections to the Feynman graphs where a gluon $g$ and an electroweak boson $V$ ( outgoing momenta $q_{1}$ and $q_{2}$ ) are attached to the same fermion line. The kinematics is given by

$$
Q\left(k_{1}\right) \rightarrow Q\left(k_{2}\right)+g\left(q_{1}\right)+V\left(q_{2}\right)
$$

where $k_{1}^{2}=k_{2}^{2}=q_{1}^{2}=0$ and momentum conservation reads $k_{1}=k_{2}+q_{2}+q_{1}$. As in 
Ref. [27], it is convenient to use the Mandelstam variables for a $2 \rightarrow 2$ process which is taken to be $\bar{q} q \rightarrow g V$. The Mandelstam variables are thus defined as

$$
\begin{aligned}
& s=\left(k_{1}-k_{2}\right)^{2}=\left(q_{1}+q_{2}\right)^{2}, \\
& t=\left(k_{1}-q_{1}\right)^{2}=\left(k_{2}+q_{2}\right)^{2}, \\
& u=\left(k_{1}-q_{2}\right)^{2}=\left(k_{2}+q_{1}\right)^{2} .
\end{aligned}
$$

The gluon polarization denoted by $\epsilon_{1}\left(q_{1}\right)$ is transverse, i.e. $\epsilon_{1} \cdot q_{1}=0$ and this permits simplifications in the virtual amplitudes. The electroweak boson $V$ is always virtual in the calculation. Its effective polarization vector $\epsilon_{2}^{\mu}\left(q_{2}\right)$ corresponds to the tree level fermion current $h^{\mu}$. Due to the emission of the Higgs boson off the $t$-channel vector boson propagator, this fermion current is not conserved. Hence, terms with $\epsilon_{2} \cdot q_{2}$ must be kept. However, electroweak gauge invariance of the amplitude is preserved, i.e., $\mathcal{M}_{\mu} q_{2}^{\mu}=0$. We have computed the virtual amplitude in the conventional dimensional regularization scheme (CDR) and used Passarino-Veltman reduction in $d=4-2 \epsilon$ spacetime dimensions to reduce tensor loop integrals into scalar loop integrals 28]. The virtual amplitude $\mathcal{M}_{V}=\mathcal{M}_{V}\left(k_{2}, q_{1}, q_{2} ; \epsilon_{1}, \epsilon_{2}\right)$ for $Q\left(k_{1}\right) \rightarrow Q\left(k_{2}\right) g\left(q_{1}\right) V\left(q_{2}\right)$ is

$$
\begin{aligned}
\mathcal{M}_{V} & =\mathcal{M}_{B} \frac{\alpha_{s}\left(\mu_{R}^{2}\right)}{4 \pi} \frac{1}{\Gamma(1-\epsilon)}\left\{\frac{1}{2}\left(\left(\frac{4 \pi \mu_{R}^{2}}{-u}\right)^{\epsilon}+\left(\frac{4 \pi \mu_{R}^{2}}{-t}\right)^{\epsilon}\right)\left(-\frac{C_{A}}{\epsilon^{2}}-\frac{\gamma_{g}}{\epsilon}\right)\right. \\
& +\frac{1}{2} \frac{C_{A}}{C_{F}}\left(\left(\frac{4 \pi \mu_{R}^{2}}{-u}\right)^{\epsilon}+\left(\frac{4 \pi \mu_{R}^{2}}{-t}\right)^{\epsilon}-2\left(\frac{4 \pi \mu_{R}^{2}}{-s}\right)^{\epsilon}\right)\left(-\frac{C_{F}}{\epsilon^{2}}-\frac{\gamma_{q}}{\epsilon}\right) \\
& \left.+2\left(\frac{4 \pi \mu_{R}^{2}}{-s}\right)^{\epsilon}\left(-\frac{C_{F}}{\epsilon^{2}}-\frac{\gamma_{q}}{\epsilon}\right)+F(-s,-t,-u)-\frac{\pi^{2}}{6} C_{A}-8 C_{F}\right\} \\
& +\widetilde{\mathcal{M}}_{V}
\end{aligned}
$$

where

$$
\begin{gathered}
F(-s,-t,-u)=\frac{C_{A}}{2}\left(\ln ^{2}\left(\frac{-u}{\mu_{R}^{2}}\right)+\ln ^{2}\left(\frac{-t}{\mu_{R}^{2}}\right)\right)-\frac{1}{2}\left(C_{A}-2 C_{F}\right) \ln ^{2}\left(\frac{-s}{\mu_{R}^{2}}\right) \\
+\frac{3}{2}\left(C_{A}-2 C_{F}\right) \ln \left(\frac{-s}{\mu_{R}^{2}}\right)+\left(\frac{1}{3} T_{R} N_{f}-\frac{5}{3} C_{A}\right)\left(\ln \left(\frac{-u}{\mu_{R}^{2}}\right)+\ln \left(\frac{-t}{\mu_{R}^{2}}\right)\right) .
\end{gathered}
$$

The finite part $\widetilde{\mathcal{M}}_{V}=\widetilde{\mathcal{M}}_{V}\left(k_{2}, q_{1}, q_{2} ; \epsilon_{1}, \epsilon_{2}\right)$ is given by

$$
\begin{aligned}
\widetilde{\mathcal{M}}_{V} & =\frac{\alpha_{s}\left(\mu_{R}^{2}\right)}{4 \pi}(-e) g_{\tau}^{V Q_{2} Q_{1}} g_{s} \\
& \cdot\left\{\left(C_{F}-\frac{1}{2} C_{A}\right)\left\{\widetilde{\mathcal{M}}_{\tau}^{(1)}\left(k_{2}, q_{1}, q_{2} ; \epsilon_{1}, \epsilon_{2}\right)+\widetilde{\mathcal{M}}_{\tau}^{(2)}\left(k_{2}, q_{1}, q_{2} ; \epsilon_{1}, \epsilon_{2}\right)\right\}\right. \\
& \left.-\frac{1}{2} C_{A} \widetilde{\mathcal{M}}_{\tau}^{(3)}\left(k_{2}, q_{1}, q_{2} ; \epsilon_{1}, \epsilon_{2}\right)\right\} .
\end{aligned}
$$

Results for physical kinematic regions can be obtained through the analytic continuation of Eq. (2.23) by the replacement of the time-like invariant by $s \rightarrow s+i 0^{+}, t \rightarrow t+i 0^{+}$, or $u \rightarrow u+i 0^{+}$. The $i \pi$ factors which result from the analytic continuation vanish upon 
interfering the virtual amplitude with the Born amplitude. The analytic continuation for any double logarithms is dealt with automatically by the fortran code for the finite part of the virtual amplitude $\widetilde{\mathcal{M}}_{V}$ given by Eq. (2.25).

The $\widetilde{\mathcal{M}}_{\tau}^{(i)}$ for $i=1,2,3$ are finite and can be expressed in terms of the finite parts of the Passarino-Veltman, $B_{0}, C_{0}$, and $D_{i j}$ functions, which we denote as $\widetilde{B}_{0}, \widetilde{C}_{0}$, and $\widetilde{D}_{i j}$. Analytic expressions for the $\widetilde{\mathcal{M}}_{\tau}^{(i)}$ are given in Appendix A.

We can build the virtual color subamplitudes of Eq. (2.18) out of the two classes of virtual corrections discussed above. The virtual color subamplitudes are then

$$
\begin{aligned}
\mathcal{A}_{3}^{\text {virt }}\left(1_{q}, 3_{g}, a_{q} ; 2_{Q}, b_{Q}\right) & =\mathcal{M}_{B}\left(p_{1}, p_{3}, p_{a 13} ; \epsilon_{3}, h_{\text {virt }}\left(p_{b} \tau_{b}, p_{2} \tau_{2}\right)\right) \\
& +\mathcal{M}_{V}\left(p_{1}, p_{3}, p_{a 13} ; \epsilon_{3}, h\left(p_{b} \tau_{b}, p_{2} \tau_{2}\right)\right), \\
\mathcal{A}_{3}^{\text {virt }}\left(2_{Q}, 3_{g}, b_{Q} ; 1_{q}, a_{q}\right) & =\mathcal{M}_{B}\left(p_{2}, p_{3}, p_{b 23} ; \epsilon_{3}, h_{\mathrm{virt}}\left(p_{a} \tau_{a}, p_{1} \tau_{1}\right)\right) \\
& +\mathcal{M}_{V}\left(p_{2}, p_{3}, p_{b 23} ; \epsilon_{3}, h\left(p_{a} \tau_{a}, p_{1} \tau_{1}\right)\right) .
\end{aligned}
$$

For the following we adopt the following abbreviations,

$$
\begin{array}{ll}
\mathcal{A}_{3,1 a}=\mathcal{A}_{3}\left(1_{q}, 3_{g}, a_{q} ; 2_{Q}, b_{Q}\right), & \mathcal{A}_{3,2 b}=\mathcal{A}_{3}\left(2_{Q}, 3_{g}, b_{Q} ; 1_{q}, a_{q}\right) \\
\mathcal{A}_{3,1 a}^{\text {virt }}=\mathcal{A}_{3}^{\text {virt }}\left(1_{q}, 3_{g}, a_{q} ; 2_{Q}, b_{Q}\right), & \mathcal{A}_{3,2 b}^{\text {virt }}=\mathcal{A}_{3}^{\text {virt }}\left(2_{Q}, 3_{g}, b_{Q} ; 1_{q}, a_{q}\right) .
\end{array}
$$

The color decomposed interference of the Born and virtual subamplitudes is then,

$$
\begin{aligned}
\overline{\sum_{\text {colors }} 2 \operatorname{Re}\left[\mathcal{A}_{3,1 a}^{\text {virt }} \mathcal{A}_{3,1 a}^{*}\right]}= & \left|\mathcal{A}_{3,1 a}\right|^{2} \frac{\alpha_{s}\left(\mu_{R}^{2}\right)}{2 \pi} \frac{1}{\Gamma(1-\epsilon)} \\
& \cdot\left\{\frac{1}{2}\left(\left(\frac{4 \pi \mu_{R}^{2}}{s_{13}}\right)^{\epsilon}+\left(\frac{4 \pi \mu_{R}^{2}}{s_{a 3}}\right)^{\epsilon}\right)\left(-\frac{C_{A}}{\epsilon^{2}}-\frac{\gamma_{g}}{\epsilon}\right)\right. \\
& +\frac{1}{2} \frac{C_{A}}{C_{F}}\left(\left(\frac{4 \pi \mu_{R}^{2}}{s_{23}}\right)^{\epsilon}+\left(\frac{4 \pi \mu_{R}^{2}}{s_{a 3}}\right)^{\epsilon}-2\left(\frac{4 \pi \mu_{R}^{2}}{s_{a 1}}\right)^{\epsilon}\right)\left(-\frac{C_{F}}{\epsilon^{2}}-\frac{\gamma_{q}}{\epsilon}\right) \\
& +2\left(\left(\frac{4 \pi \mu_{R}^{2}}{s_{a 1}}\right)^{\epsilon}+\left(\frac{4 \pi \mu_{R}^{2}}{s_{b 2}}\right)^{\epsilon}\right)\left(-\frac{C_{F}}{\epsilon^{2}}-\frac{\gamma_{q}}{\epsilon}\right) \\
& \left.-\frac{\pi^{2}}{6} C_{A}-16 C_{F}+F\left(s_{a 1}, s_{a 3}, s_{13}\right)\right\} \\
& +2 \operatorname{Re}\left[\widetilde{\mathcal{A}}_{3}^{\text {virt }}\left(1_{q}, 3_{g}, a_{q} ; 2_{Q}, b_{Q}\right) \mathcal{A}_{3,1 a}^{*}\right]
\end{aligned}
$$

with $\widetilde{\mathcal{A}}_{3}^{\text {virt }}\left(1_{q}, 3_{g}, a_{q} ; 2_{Q}, b_{Q}\right)=\widetilde{\mathcal{M}}_{V}\left(p_{1}, p_{3}, p_{a 13} ; \epsilon_{3}, h\left(p_{b} \tau_{b}, p_{2} \tau_{2}\right)\right)$. A similar expression for $2 \operatorname{Re}\left[\mathcal{A}_{3,2 b}^{\text {virt }} \mathcal{A}_{3,2 b}^{*}\right]$ is obtained by making the replacements, $a \leftrightarrow b$ and $1 \leftrightarrow 2$, in Eq. (2.29).

Summing together the contributions from Eq. (2.15) and Eq. (2.29) yields the finite 3-parton NLO cross section

$$
\begin{aligned}
\sigma_{3}^{N L O}(q Q \rightarrow q Q g H) & =\int_{0}^{1} d x_{a} \int_{0}^{1} d x_{b} f_{q / p}\left(x_{a}, \mu_{F}\right) f_{Q / p}\left(x_{b}, \mu_{F}\right) \\
& \times \frac{1}{2 \hat{s}} d \Phi_{4}\left(p_{a}, p_{b}\right) F_{J}^{(3)}\left(p_{1}, p_{2}, p_{3}, P ; p_{a}, p_{b}\right) \\
& \cdot\left\{\left|\mathcal{M}_{B}\left(1_{q}, 2_{Q}, 3_{g}, a_{q}, b_{Q}\right)\right|^{2}\left(1+\frac{\alpha_{s}\left(\mu_{R}^{2}\right)}{2 \pi} K_{\mathrm{born}}\right)\right.
\end{aligned}
$$




$$
\begin{aligned}
& +\left|\mathcal{A}_{3}\left(1_{q}, 3_{g}, a_{q} ; 2_{Q}, b_{Q}\right)\right|^{2} \frac{\alpha_{s}\left(\mu_{R}^{2}\right) C_{F}}{2 \pi} F\left(s_{a 1}, s_{a 3}, s_{13}\right) \\
& +\left|\mathcal{A}_{3}\left(2_{Q}, 3_{g}, b_{Q} ; 1_{q}, a_{q}\right)\right|^{2} \frac{\alpha_{s}\left(\mu_{R}^{2}\right) C_{F}}{2 \pi} F\left(s_{b 2}, s_{b 3}, s_{23}\right) \\
& +C_{F}\left(2 \operatorname{Re}\left[\widetilde{\mathcal{A}}_{3}^{\text {virt }}\left(1_{q}, 3_{g}, a_{q} ; 2_{Q}, b_{Q}\right) \mathcal{A}_{3}^{*}\left(1_{q}, 3_{g}, a_{q} ; 2_{Q}, b_{Q}\right)\right]\right. \\
& \left.\left.+2 \operatorname{Re}\left[\widetilde{\mathcal{A}}_{3}^{\text {virt }}\left(2_{Q}, 3_{g}, b_{Q} ; 1_{q}, a_{q}\right) \mathcal{A}_{3}^{*}\left(2_{Q}, 3_{g}, b_{Q} ; 1_{q}, a_{q}\right)\right]\right)\right\}
\end{aligned}
$$

with

$$
K_{\text {born }}=\left(-\frac{2 \pi^{2}}{3}+\frac{50}{9}\right) C_{A}-\frac{16}{9} T_{R} N_{f}+2 C_{F}\left(2-\pi^{2}\right) .
$$

The remaining divergent piece of the integral of the dipole factors in Eq. (2.12) is proportional to the $P^{q q}$ and $P^{g q}$ splitting functions and is factorized into the parton distribution functions. The surviving finite collinear terms are given by

$$
\begin{aligned}
\sigma_{3, \mathrm{col}}^{N L O}(q Q \rightarrow q Q g H)= & \int_{0}^{1} d x_{a} \int_{0}^{1} d x_{b} \frac{1}{2 \hat{s}} d \Phi_{4}\left(p_{a}, p_{b}\right) F_{J}^{(3)}\left(p_{1}, p_{2}, p_{3} ; p_{a}, p_{b}\right) \\
& \cdot\left\{\left(f_{q / p}\left(x_{a}, \mu_{F}\right) f_{Q / p}^{2, b}\left(x_{b}, \mu_{F}, \mu_{R}\right)+f_{q / p}^{1, a}\left(x_{a} ; \mu_{F}, \mu_{R}\right) f_{Q / p}\left(x_{b} ; \mu_{F}\right)\right)\right. \\
& \cdot\left|\mathcal{M}_{3}\left(1_{q}, 2_{Q}, 3_{g} ; a_{q}, b_{Q}\right)\right|^{2} \\
+ & \frac{1}{2} C_{A} f_{q / p}\left(x_{a} ; \mu_{F}\right)\left(f_{Q / p}^{3, b}\left(x_{b} ; \mu_{F}, \mu_{R}\right)-f_{Q / p}^{2, b}\left(x_{b} ; \mu_{F}, \mu_{R}\right)\right) \\
& \cdot\left|\mathcal{A}_{3}\left(2_{Q}, 3_{g}, b_{Q} ; 1_{q}, a_{q}\right)\right|^{2} \\
& +\frac{1}{2} C_{A}\left(f_{q / p}^{3, b}\left(x_{a} ; \mu_{F}, \mu_{R}\right)-f_{q / p}^{1, a}\left(x_{a} ; \mu_{F}, \mu_{R}\right)\right) f_{Q / p}\left(x_{b}, \mu_{F}\right) \\
& \left.\cdot\left|\mathcal{A}_{3}\left(1_{q}, 3_{g}, a_{q} ; 2_{Q}, b_{Q}\right)\right|^{2}\right\},
\end{aligned}
$$

and similarly for the anti-quark initiated processes. Here the quark functions $f_{q / p}^{i, j}\left(x ; \mu_{F}, \mu_{R}\right)$ are given by

$$
\begin{aligned}
f_{q / p}^{i, j}\left(x ; \mu_{F}, \mu_{R}\right) & =\frac{\alpha_{s}\left(\mu_{R}\right)}{2 \pi} \int_{x}^{1} \frac{d z}{z}\left\{f_{g / p}\left(\frac{x}{z} ; \mu_{F}\right) A_{g q}^{i, j}(z)\right. \\
& +\left[f_{q / p}\left(\frac{x}{z} ; \mu_{F}\right)-z f_{q / p}\left(x ; \mu_{F}\right)\right] B_{q q}^{i, j}(z) \\
& \left.+f_{q / p}\left(\frac{x}{z} ; \mu_{F}\right) C_{q q}^{i, j}(z)\right\}+\frac{\alpha_{s}\left(\mu_{R}\right)}{2 \pi} f_{q / p}\left(x ; \mu_{F}\right) D_{q q}^{i, j}(x),
\end{aligned}
$$

with kernels

$$
\begin{aligned}
A_{g q}^{i, j}(z) & =T_{R}\left[z^{2}+(1-z)^{2}\right] \ln \frac{2 p_{j} p_{i}(1-z)}{\mu_{F}^{2} z}+T_{R} 2 z(1-z) . \\
B_{q q}^{i, j}(z) & =C_{F}\left[\frac{2}{1-z} \ln \frac{2 p_{j} p_{i}(1-z)}{\mu_{F}^{2}}-\frac{\gamma_{i}}{C_{i}} \frac{1}{1-z}\right], \\
C_{q q}^{i, j}(z) & =C_{F}\left[-(1+z) \ln \frac{2 p_{j} p_{i}(1-z)}{\mu_{F}^{2} z}-\frac{2}{1-z} \ln z+(1-z)\right], \\
D_{q q}^{i, j}(x) & =C_{F}\left[\frac{2 \pi^{2}}{3}-5-\frac{\gamma_{i}}{C_{i}}-\frac{\gamma_{i}}{C_{i}} \ln (1-x)+\ln ^{2}(1-x)\right. \\
& \left.+\frac{3}{2} \ln \frac{2 p_{i} p_{j}}{\mu_{F}^{2}}+2 \ln (1-x) \ln \frac{2 p_{i} p_{j}}{\mu_{F}^{2}}\right] .
\end{aligned}
$$




\begin{tabular}{cccccc}
\hline \hline$\alpha_{s}^{N L O}\left(M_{Z}\right)$ & $\alpha_{s}^{L O}\left(M_{Z}\right)$ & $M_{Z}$ & $M_{W}$ & $G_{F}$ & $m_{h}$ \\
0.118 & 0.130 & $91.188 \mathrm{GeV}$ & $80.416 \mathrm{GeV}$ & $1.16639 \times 10^{-5} / \mathrm{GeV}^{2}$ & $120 \mathrm{GeV}$ \\
\hline \hline
\end{tabular}

Table 2: Standard Model input parameters

where if parton $i$ is a gluon, $C_{i}=C_{A}$ and if parton $i$ is a quark or anti-quark, $C_{i}=C_{F}$. Likewise, $\gamma_{i}=\gamma_{q}$ if parton $i$ is a quark or anti-quark and $\gamma_{i}=\gamma_{g}$ if parton $i$ is a gluon. The analogous results for gluon initiated processes are given in Appendix B.

We have implemented the QCD corrections for $p p \rightarrow H j j j$ into a fully flexible partonlevel Monte Carlo program. We have checked the dipole subtraction by verifying that the dipole subtraction terms and the real emission matrix elements match in the various singular regions. The gauge invariance of the virtual matrix elements has been checked numerically for random choices of momenta. The finite collinear counter-terms that remain after the factorization of initial-state collinear divergences have been obtained by two independent calculations. We have also introduced a cut, $\alpha \in(0,1]$, on the phase space of the dipoles as described in Ref. [29]. We have checked that the integrated cross section is independent of this parameter and have used $\alpha=0.3$ in our simulations.

In all subsequent calculations we use the input parameters for defining Standard Model (SM) couplings as listed in Table 2. Other SM couplings are computed using LO electroweak relations. Cross sections are computed using CTEQ6M parton distributions 30 for all NLO results and CTEQ6L1 parton distributions for all leading order cross sections. The running of the strong coupling is evaluated at two-loop order, with $\alpha_{s}\left(M_{Z}\right)=0.118$, for all NLO results. For LO results, the running of the strong coupling is evaluated at one-loop with $\alpha_{s}\left(M_{Z}\right)=0.130$. In order to reconstruct jets from the final-state partons, the $k_{T}$ algorithm [31] as described in Ref. [32] is used, with resolution parameter $D=0.8$. 


\section{Predictions for the LHC}

The goal of our calculation is a precise prediction of the LHC cross section for Higgs boson production in VBF with three or more jets. The $k_{T}$ algorithm is used to define jets and these jets are required to have

$$
p_{T j} \geq 20 \mathrm{GeV}, \quad\left|y_{j}\right| \leq 4.5 .
$$

Here $y_{j}$ denotes the rapidity of the (massive) jet momentum which is reconstructed as the four-vector sum of massless partons of pseudorapidity $|\eta|<5$.

At LO, there are exactly three massless final state partons. At NLO these jets may be composed of two partons (recombination effect) or four well-separated partons may be encountered, of which at least three satisfy the cuts of Eq. (3.1) and would give rise to either three or four-jet events. As with LHC data, a choice needs to be made for selecting the tagging jets in such a multijet situation. Here the " $p_{T}$-method" is chosen. For a given event, the tagging jets are defined as the two jets with the highest transverse momentum with

$$
p_{T j}^{\mathrm{tag}} \geq 30 \mathrm{GeV}, \quad\left|y_{j}^{\mathrm{tag}}\right| \leq 4.5 .
$$

The non-tagging jets by default are jets of lowest transverse momenta. They do not need to satisfy the cuts of Eq. (3.2) but must satisfy the cuts of Eq. (3.1).

The Higgs boson decay products (generically called "leptons" in the following) are required to fall between the two tagging jets in rapidity and they should be well observable. While the exact criteria for the Higgs decay products will depend on the channel considered, such specific requirements here are substituted by generating isotropic Higgs boson decay into two massless "leptons" (which represent $\tau^{+} \tau^{-}$or $\gamma \gamma$ final states) and requiring

$$
p_{T \ell} \geq 20 \mathrm{GeV}, \quad\left|\eta_{\ell}\right| \leq 2.5, \quad \triangle R_{j \ell} \geq 0.6,
$$

where $\triangle R_{j \ell}$ denotes the jet-lepton separation in the rapidity-azimuthal angle plane. In addition, the two "leptons" are required to fall between the two tagging jets in rapidity:

$$
y_{j, \min }^{\mathrm{tag}}+0.6<\eta_{\ell_{1,2}}<y_{j, \max }^{\mathrm{tag}}-0.6 .
$$

Note that no reduction due to branching ratios for specific final states has been included in the calculation.

Backgrounds to vector-boson fusion are significantly suppressed by requiring a large rapidity separation for the two tagging jets. Tagging jets are required to reside in opposite detector hemispheres with

$$
y_{j}^{\operatorname{tag} 1} \cdot y_{j}^{\operatorname{tag} 2}<0
$$

and to have a large rapidity separation of

$$
\Delta y_{j j}=\left|y_{j}^{\operatorname{tag} 1}-y_{j}^{\operatorname{tag} 2}\right|>4,
$$

sometimes called "rapidity gap cut". QCD backgrounds for the Higgs signal typically occur at small invariant masses, due to a the dominance of gluons at small Feynman $x$ in the 
incoming protons [8]. The QCD backgrounds can be reduced by imposing a lower bound on the invariant mass of the tagging jets of

$$
m_{j j}=\sqrt{\left(p_{j}^{\operatorname{tag} 1}+p_{j}^{\operatorname{tag} 2}\right)^{2}}>600 \mathrm{GeV} .
$$

The cross section for Higgs production via VBF in association with three jets or more (Hjjj), within the cuts of Eqs. (3.1)-(3.7), is shown in Fig. [7. The scale dependence of the NLO and LO cross sections is shown for factorization and renormalization scales, $\mu_{F}$ and $\mu_{R}$, which are tied to a fixed reference scale $\mu_{0}=40 \mathrm{GeV}$,

$$
\mu_{R}=\xi_{R} \mu_{0}, \quad \mu_{F}=\xi_{F} \mu_{0} .
$$

The value $\mu_{0}=40 \mathrm{GeV}$ was chosen to minimize the scale dependence of the NLO predictions and at the same time it provides optimal agreement of the LO approximation with the NLO result.

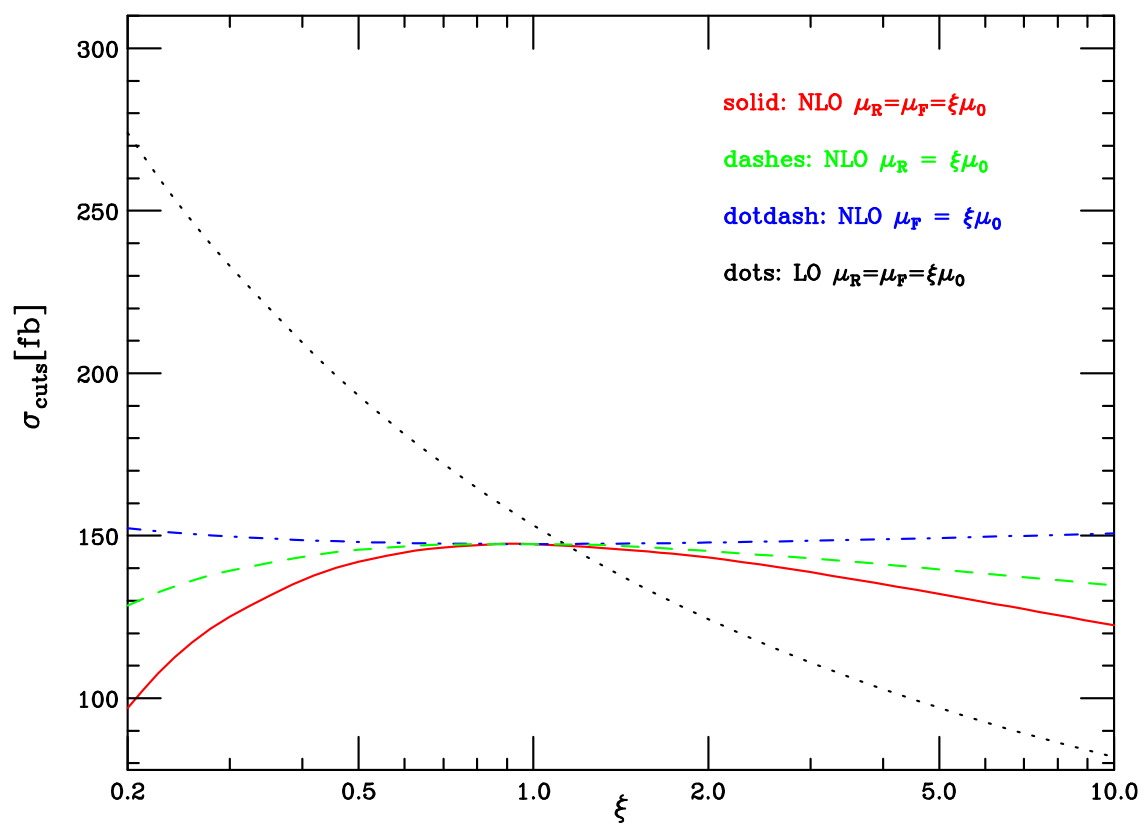

Figure 7: Scale dependence of the total cross section at LO and NLO within the cuts of Eqs. (3.1)(3.7) for VBF $H j j j$ production at the LHC. The factorization scale $\mu_{F}$ and the renormalization scale $\mu_{R}$ are taken as multiples, $\xi \mu_{0}$, of the fixed reference scale $\mu_{0}=40 \mathrm{GeV}$. The NLO curves are for $\mu_{R}=\mu_{F}=\xi \mu_{0}$ (solid red line), $\mu_{F}=\mu_{0}$ and $\mu_{R}=\xi \mu_{0}$ (dashed green line), and $\mu_{F}=\xi \mu_{0}$ and $\mu_{R}=\xi \mu_{0}$ (dot-dashed blue line ). The dotted black curve shows the scale dependence of the LO cross section for $\mu_{R}=\mu_{F}=\xi \mu_{0}$.

The LO cross section depends on both the factorization and renormalization scale. For $\mu_{R}=\mu_{F}=\xi \mu_{0}$ with $0.5<\xi<2$ the scale variation is $+26 \%$ to $-19 \%$ for the LO cross section. The large scale variation is primarily due the fact that the LO Hjjj production cross section is proportional to $\alpha_{s}$. This is in contrast to $H j j$ production in VBF, which only depends on the factorization scale at LO. At NLO three choices are shown: (a) $\xi_{R}=\xi_{F}=\xi$ (solid red line); (b) $\xi_{R}=\xi, \xi_{F}=1$ (dashed green line); (c) $\xi_{R}=1$, 
$\xi_{F}=\xi$ (dot-dashed blue line). Allowing for a factor 2 variation in either direction, i.e., considering the range $0.5 \leq \xi \leq 2$, the NLO cross section changes by less than $5 \%$ in all cases.
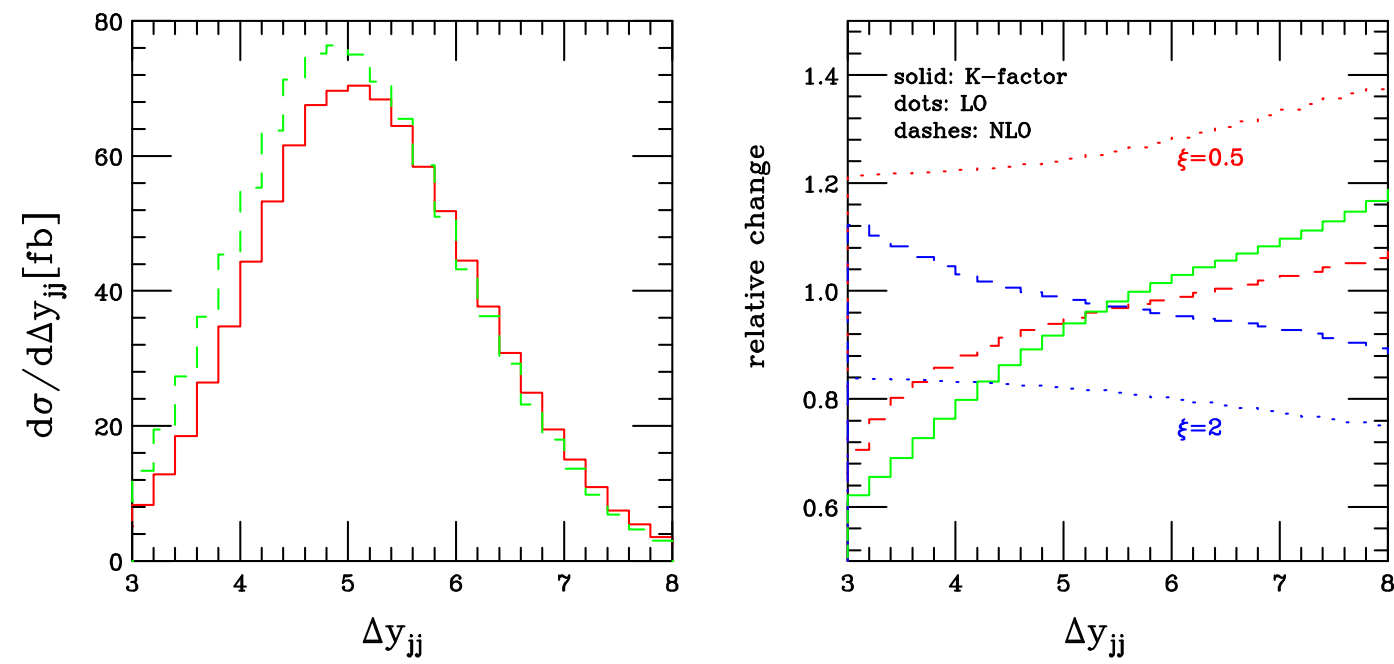

Figure 8: Rapidity separation in $H j j j$ production within the cuts of Eqs. (3.1)-(3.5) and Eq. (3.7). In the left panel, $d \sigma / d \Delta y_{j j}$ is shown at LO (dashed green) and NLO (solid red) for $\mu_{F}=\mu_{R}=$ $\mu_{0}=40 \mathrm{GeV}$. The right-hand panel depicts the $K$ factor (solid green) and scale variations of LO (dotted) and NLO (dashed) results for $\mu_{R}=\mu_{F}=\xi \mu_{0}$ with $\xi=1 / 2$ and 2.

Our Monte Carlo program allows the analysis of arbitrary infrared and collinear safe distributions with NLO QCD accuracy. In order to assess the impact of the NLO corrections we compare LO and NLO results by plotting the dynamical $K$ factor

$$
K(x)=\frac{d \sigma_{3}^{N L O}\left(\mu_{R}=\mu_{F}=\mu_{0}\right) / d x}{d \sigma_{3}^{L O}\left(\mu_{R}=\mu_{F}=\mu_{0}\right) / d x}
$$

for our fixed reference scale of $\mu_{0}=40 \mathrm{GeV}$. The stability of the results is represented via the scale dependence, given by the ratio of cross sections and dubbed "relative change" in the following,

$$
\text { relative change }=\frac{d \sigma_{3}\left(\mu_{R}=\mu_{F}=\xi \mu_{0}\right) / d x}{d \sigma_{3}\left(\mu_{R}=\mu_{F}=\mu_{0}\right) / d x} .
$$

We plot results for $\xi=1 / 2$ and 2 with $\mu_{0}=40 \mathrm{GeV}$ for NLO and LO distributions.

The wide separation in rapidity of the tagging jets is a characteristic feature of VBF processes. In the left-hand panel of Fig. 8 the distribution $d \sigma / d \Delta y_{j j}$ is shown at LO (dashed green) and at NLO (solid red) for $H j j j$ production. Just as in the NLO Hjj case [19], the NLO corrections push the peak towards higher values of rapidity separation $\Delta y_{j j}$. This strengthens the case for the rapidity gap cut of $\Delta y_{j j}>4$. The $K$ factor (solid green) in the right-hand-side of Fig. 8 is strongly phase space dependent. The scale variations $\xi=2^{ \pm 1}$ are significantly reduced by the NLO corrections, from $\approx 25 \%$ at LO to $\approx 10 \%$ or less at NLO in the relevant region $4<\Delta y_{j j}<7$. Similar results are found for the transverse momentum distribution of the tagging jets as shown in Fig. 9 . 

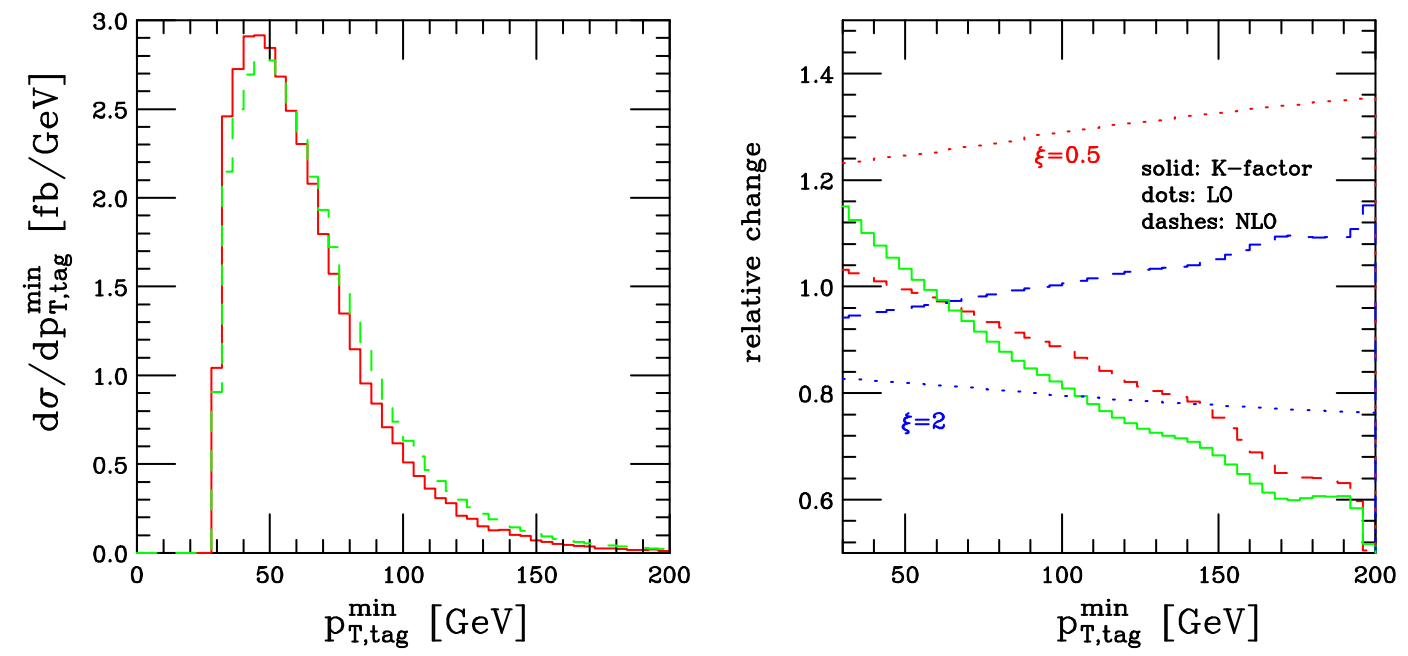

Figure 9: Transverse momentum distribution for the softer tagging jet in $\mathrm{Hjjj}$ production within the cuts of Eqs. (3.1)-(3.7). The meaning of the curves is the same as in Fig. 8.
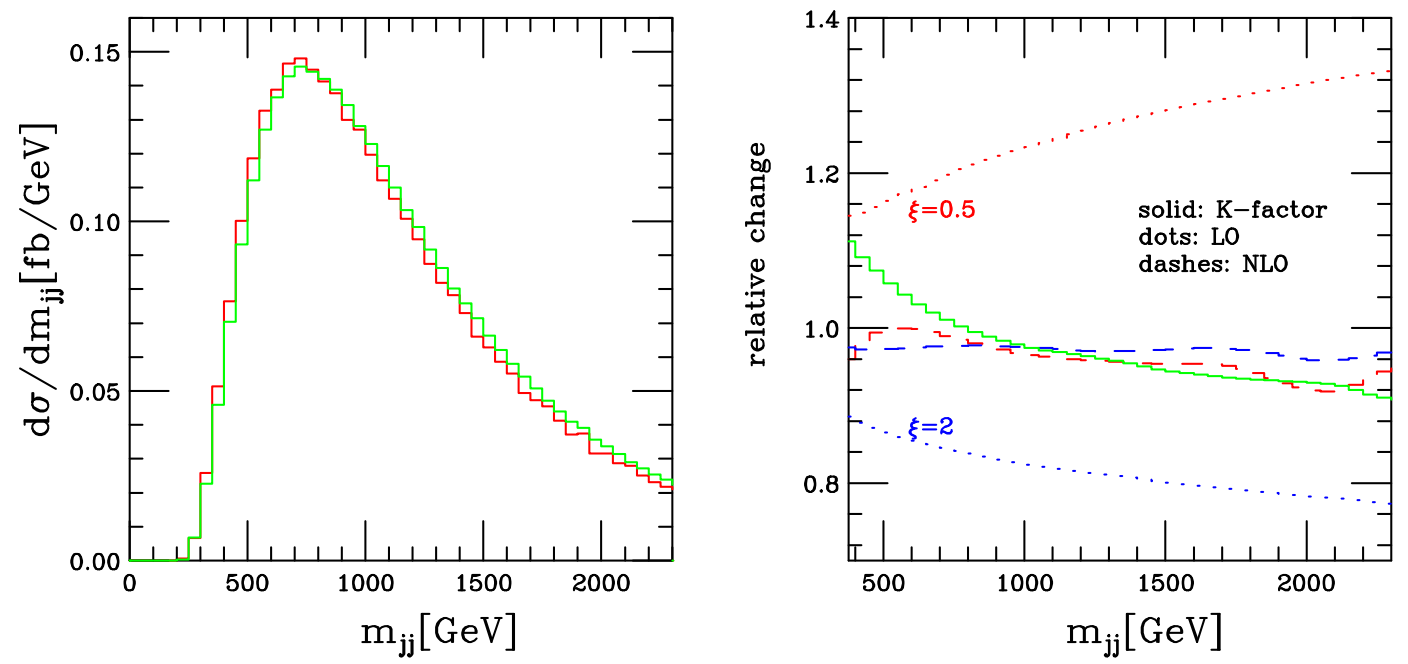

Figure 10: Invariant mass distribution of the two tagging jets in $H j j j$ production within the cuts of Eqs. (3.1)-(3.5) and Eq. (3.6). The meaning of the curves is the same as in Fig. 8.

In Fig. 10 the invariant mass distribution of the two tagging jets is shown for a fixed value of renormalization and factorization scale, $\mu_{R}=\mu_{F}=40 \mathrm{GeV}$. The $K$ factor (solid green) deviates from unity by $10 \%$ or less for this distribution and this scale choice, i.e. the LO result provides for an excellent estimate. The $\xi=2^{ \pm 1}$ scale variations produce changes in the LO distribution of about 30\%, however (dotted lines). This uncertainty is reduced to the $5 \%$ level at NLO (dashed curves).

When contemplating a central jet veto for the VBF signal, the probability for observing three (or more) jets in the final state becomes crucial. With the two leading jets defined as tagging jets, one would like to know this probability for emitting additional jets as a function of tagging jet distributions. It is given by the 3 -jet ratio $R=\sigma_{3} / \sigma_{2}$, which we 

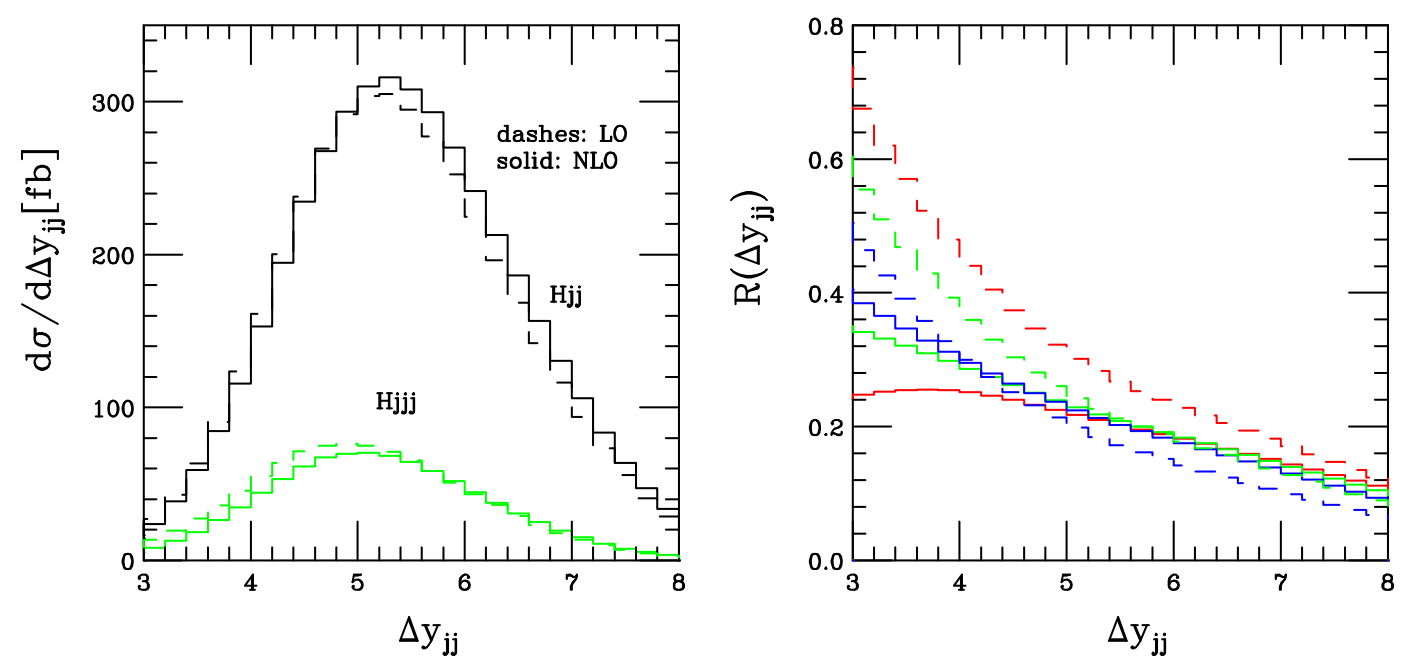

Figure 11: The rapidity separation of two tagging jets for $m_{h}=120 \mathrm{GeV}$ within the cuts of Eqs. (3.1)-(3.5) and Eq. (3.7). In the left panel, $d \sigma / d \Delta y_{j j}$ is shown at NLO (solid histograms) and LO (dashed histograms) for $H j j$ and $H j j j$ production with a fixed scale $\mu_{R}=\mu_{F}=40 \mathrm{GeV}$. In the right panel, 3-jet ratios, $R\left(\Delta y_{j j}\right)$ are shown at LO (dashed) and at NLO (solid) for $\mu_{R}=\mu_{F}=$ 20,40 , and $80 \mathrm{GeV}$.

define for arbitrary distributions as

$$
R^{\{L O, N L O\}}(x)=\frac{d \sigma_{3}^{\{L O, N L O\}}\left(\mu_{R}, \mu_{F}\right) / d x}{d \sigma_{2}^{N L O}\left(\mu_{R}=\mu_{F}=m_{h}\right) / d x} .
$$

For both NLO and LO 3-jet ratios, the distribution for Higgs plus two jet production in the denominator is computed to NLO accuracy since this provides the most accurate cross section estimate. For these $H j j$ distributions, the NLO parton-level Monte Carlo program described in 19] is used with renormalization scale and factorization scale set to the mass of the Higgs boson, $m_{h}$. The numerator corresponds to the analogous distribution for Higgs plus three jet inclusive events (VBF $H j j j$ production) for which we explore LO and NLO predictions and different scale choices.

Let us start by considering the scale variations of the 3 -jet ratio as a function of the rapidity separation of the tagging jets, $x=\Delta y_{j j}$, (in Fig. 11) and of the invariant mass, $x=m_{j j}$, of the two tagging jets (in Fig. 12). The left-hand panels show the distributions for 2-jet inclusive and 3-jet inclusive events as predicted at LO (dashed histograms) and NLO (solid histograms) for a fixed scale $\mu_{R}=\mu_{F}=40 \mathrm{GeV}$. The right-hand panels then give the corresponding 3 -jet ratios for three choices of scales, $\mu_{R}=\mu_{F}=20,40$, and $80 \mathrm{GeV}$.

The 3 -jet ratio decreases with increasing rapidity separation of the tagging jets. This is largely a kinematic effect: additional radiation in VBF events is mostly emitted outside the rapidity range set by the two tagging jets. Thus, the available phase space for additional jets diminishes rapidly as $\Delta y_{j j}$ increases. While typical 3 -jet ratios are around 0.2 , the LO ratio $R^{L O}\left(\Delta y_{j j}\right)$ (dashed curves) reaches values up to 0.7 at low values of $\Delta y_{j j}$. The corresponding NLO ratio is significantly lower, around 0.4. The reason is that at NLO the 

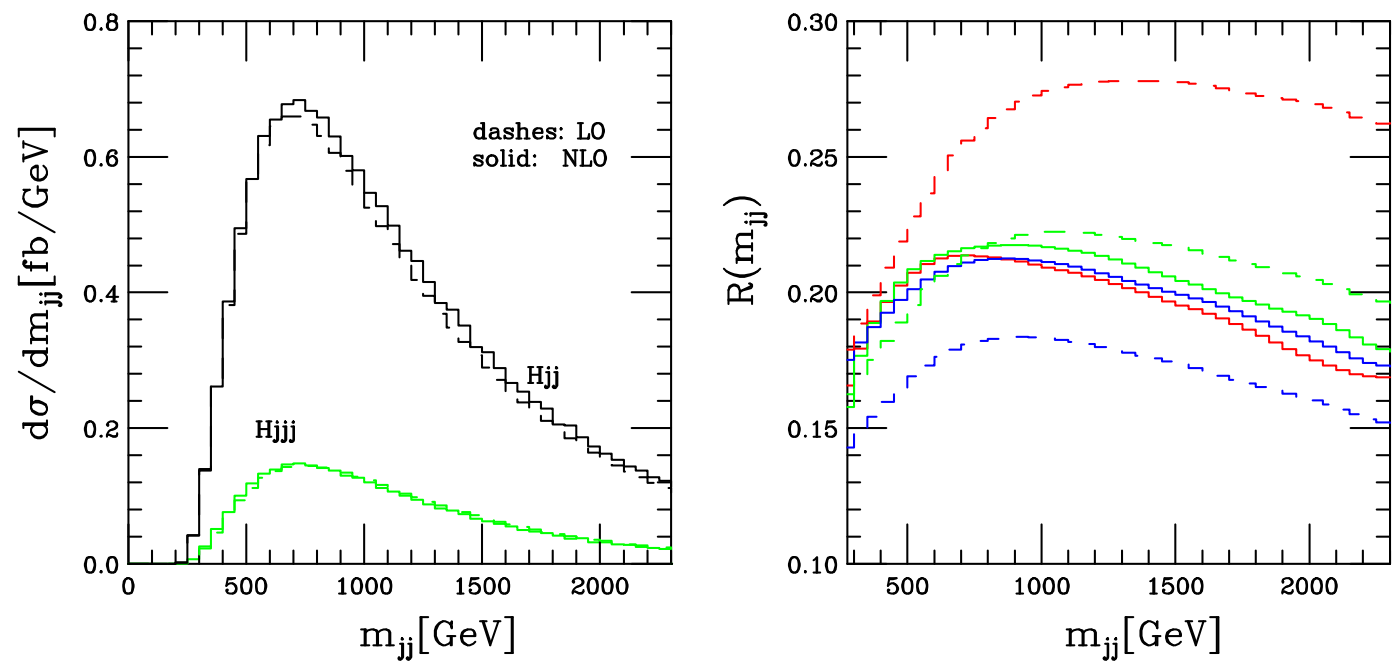

Figure 12: The dijet invariant mass of two tagging jets for $m_{h}=120 \mathrm{GeV}$ within the cuts of Eqs. (3.1)-(3.6). The meaning of the curves is the same as in Fig. 11.

separation of the tagging jets increases somewhat. When normalizing to the NLO $H j j$ cross section $R^{L O}$ is enhanced in the $\Delta y_{j j}=3$ region, where cross sections are very small due to the $m_{j j}>600 \mathrm{GeV}$ cut. There is no such effect for $R^{N L O}$. One also notices that for higher values of $\Delta y_{j j}$ the scale dependence decreases, becoming insignificant at NLO in the phase space region with typical VBF cuts $\left(\Delta y_{j j}>4\right)$.

Similar threshold effects appear in the $m_{j j}$ distributions of Fig. 12: large scale variations at NLO are confined to the low $m_{j j}$ region with negligible cross section due to the cuts. The 3-jet ratios decrease somewhat at large values of the dijet invariant mass. However, the effect is not as strong as in the $\Delta y_{j j}$ distribution. Particularly striking is the reduction of the scale uncertainty when going from $R^{L O}(\approx 30 \%)$ to $R^{N L O}$ (5 to $10 \%$ ).

Veto jets are typically defined to be non-tagging jets that reside in the rapidity region between the tagging jets. In addition to the cuts of Eqs. (3.1)-(3.7), we employ the following definition for the veto jets,

$$
p_{T j}^{\text {veto }}>p_{T, \text { veto }}, \quad y_{j}^{\text {veto }} \in\left(y_{j}^{\operatorname{tag} 1}, y_{j}^{\operatorname{tag} 2}\right) .
$$

For 4-jet events it is possible to identify two veto jets. In this case, we order the veto jets according to their transverse momentum with $p_{T j}^{\text {veto } 1}>p_{T j}^{\text {veto } 2}$. In the following we take $p_{T, \text { veto }}=20 \mathrm{GeV}$ unless stated otherwise.

On the left-hand-side of Fig. 13 the rapidity distribution, $d \sigma / d y_{r e l}$, of the highest $p_{T}$ veto jet is shown. Here the rapidity is measured with respect to the average rapidity of the tagging jets,

$$
y_{\text {rel }}=y_{j}^{\text {veto }}-\left(y_{j}^{\operatorname{tag} 1}+y_{j}^{\operatorname{tag} 2}\right) / 2 .
$$

The two histograms correspond to the LO (dashed green) and NLO (solid red) distributions at a scale $\mu_{R}=\mu_{F}=40 \mathrm{GeV}$. The suppression of jet activity in the center, near $y_{r e l}=0$, is even more pronounced at NLO than at LO, i.e. the higher order corrections strengthen the rapidity gap features of VBF events. This is reflected by the $K$ factor (solid line in 
right-side panel of Fig. 13) which is greater than one for $\left|y_{\text {rel }}\right|>2$ and is less than one in the central region between the tagging jets. The right-hand-side of the figure also shows the scale variations for $\xi=2^{ \pm 1}$ relative to the $\xi=1$ case: the scale dependence is significantly reduced at NLO (dashed curves) and remains largest in the regions of small cross section. In the vicinity of $y_{\text {rel }}=0$ the NLO result varies between $-20 \%$ and $+7 \%$ down from a LO variation of $-20 \%$ to $+24 \%$. In the large cross section regions, near $y_{\text {rel }} \approx \pm 2$, the scale variations at NLO are a few percent only, a drastic improvement from the LO situation. This small scale dependence in the large cross section region will be reflected in small QCD uncertainties at NLO for jet veto probabilities.

The effect is clearly visible in Fig. 14 where the transverse momentum distribution for the highest $p_{T}$ veto jet is shown for $\mu_{R}=\mu_{F}=40 \mathrm{GeV}$ at LO (dashed green) and NLO (solid red). The scale variations are largest at high $p_{T j}^{\text {veto }}$, but even at a value of $80 \mathrm{GeV}$ the NLO results for $\xi=2^{ \pm 1}$ (dashed curves) deviate from the $\xi=1$ case by only $-3 \%$ to $+10 \%$. At LO (dotted) these scale variations are $-22 \%$ to $+31 \%$. The $K$ factor (solid green) is close to one but decreases monotonically, i.e. at NLO the veto jet becomes slightly softer.
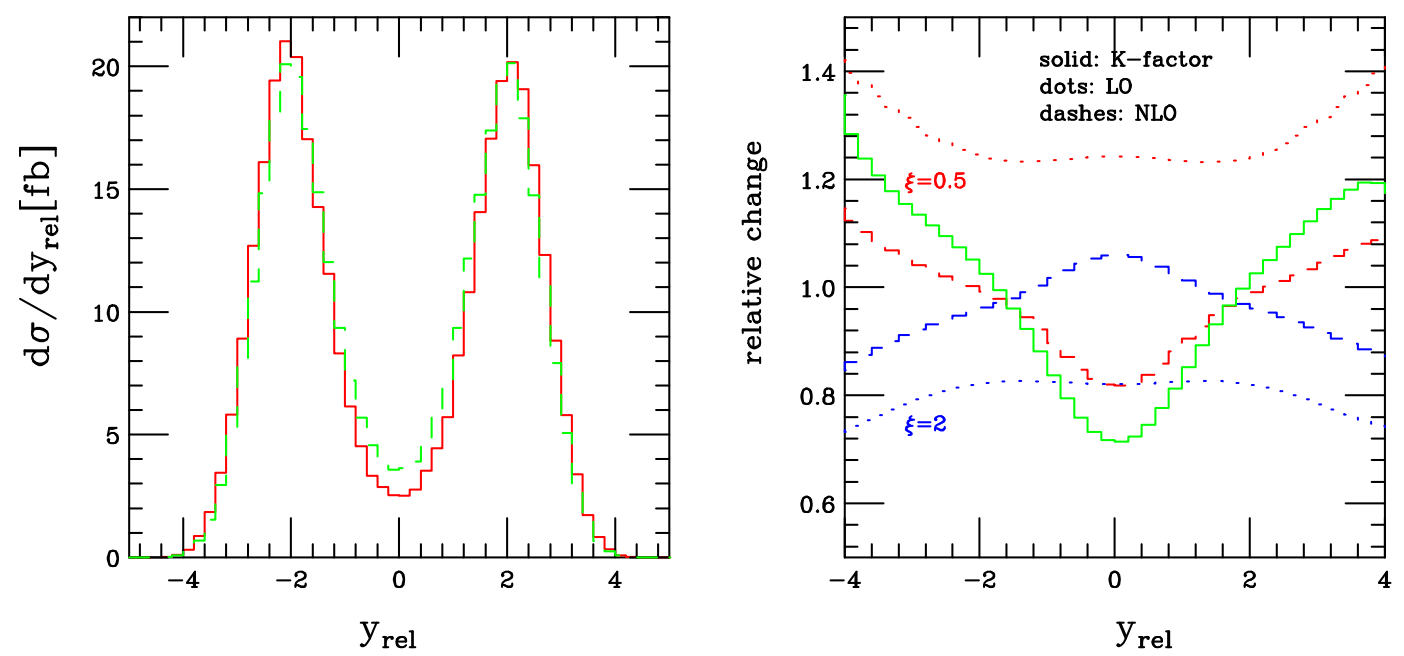

Figure 13: The distribution in rapidity of the highest $p_{T}$ veto jet with the cuts of Eqs. (3.1)- 3.7) and Eq. (3.12), measured with respect to the rapidity average of the tagging jets. In the left panel, $d \sigma / d y_{\text {rel }}$ is shown at LO (dashed green) and NLO (solid red) for $\mu_{F}=\mu_{R}=40 \mathrm{GeV}$. In the right-hand panel the $K$ factor (solid green) and scale variations of LO (dotted) and NLO (dashed) results are shown for $\mu_{R}=\mu_{F}=\xi \mu_{0}$ with $\xi=1 / 2$ and 2 .

Fig. 15 shows the effect of the veto cuts defined by Eq. (3.12) on the tagging jet invariant mass distribution. Both LO and NLO 3-jet ratios are reduced compared to Fig. 12 due to the restricted rapidity range of Eq. (3.12) for the veto jets. Fig. 16 depicts the distribution in rapidity separation of the tagging jets with veto cuts. Again, the 3-jet ratios are reduced. However, one also finds a significant shape change of the $\Delta y_{j j}$ dependence: the fairly steep decrease of the 3 -jet ratio with increasing $\Delta y_{j j}$ becomes much less pronounced at NLO. 

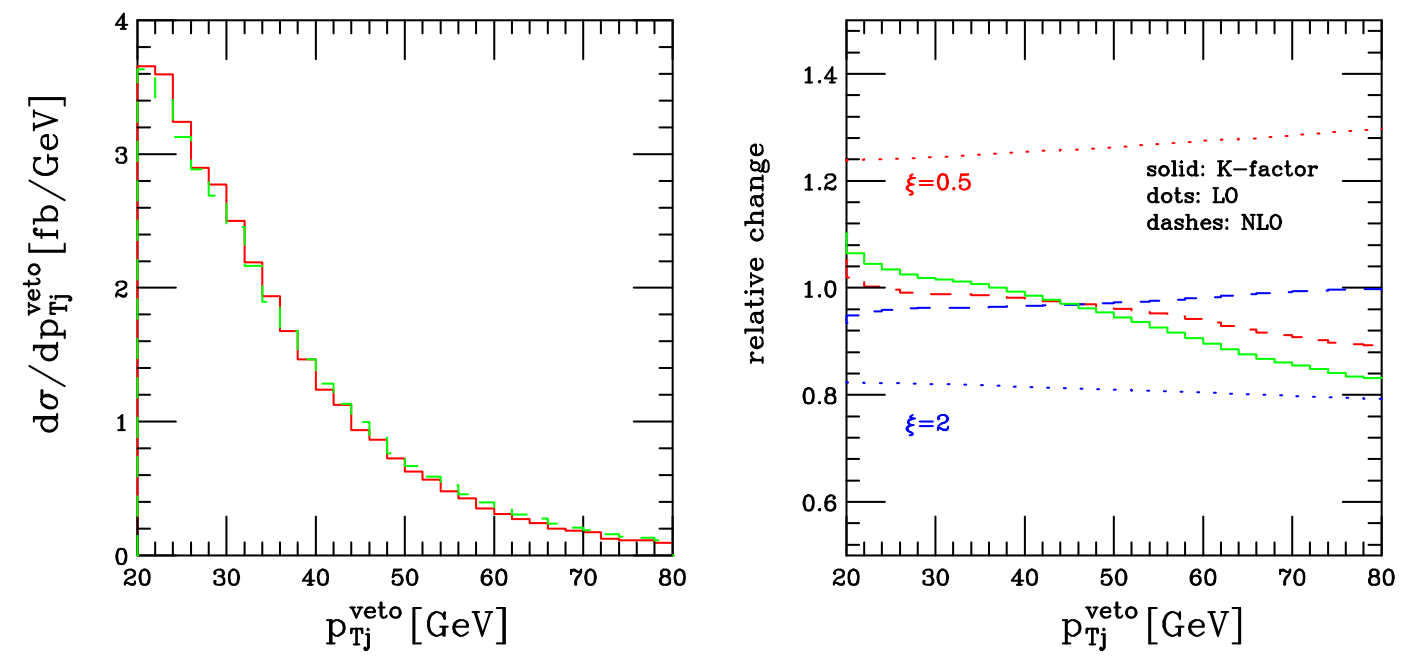

Figure 14: Same as Fig. 13 but for the distribution in the transverse momentum, $p_{T j}^{\mathrm{veto}}$, of the highest $p_{T}$ veto jet.
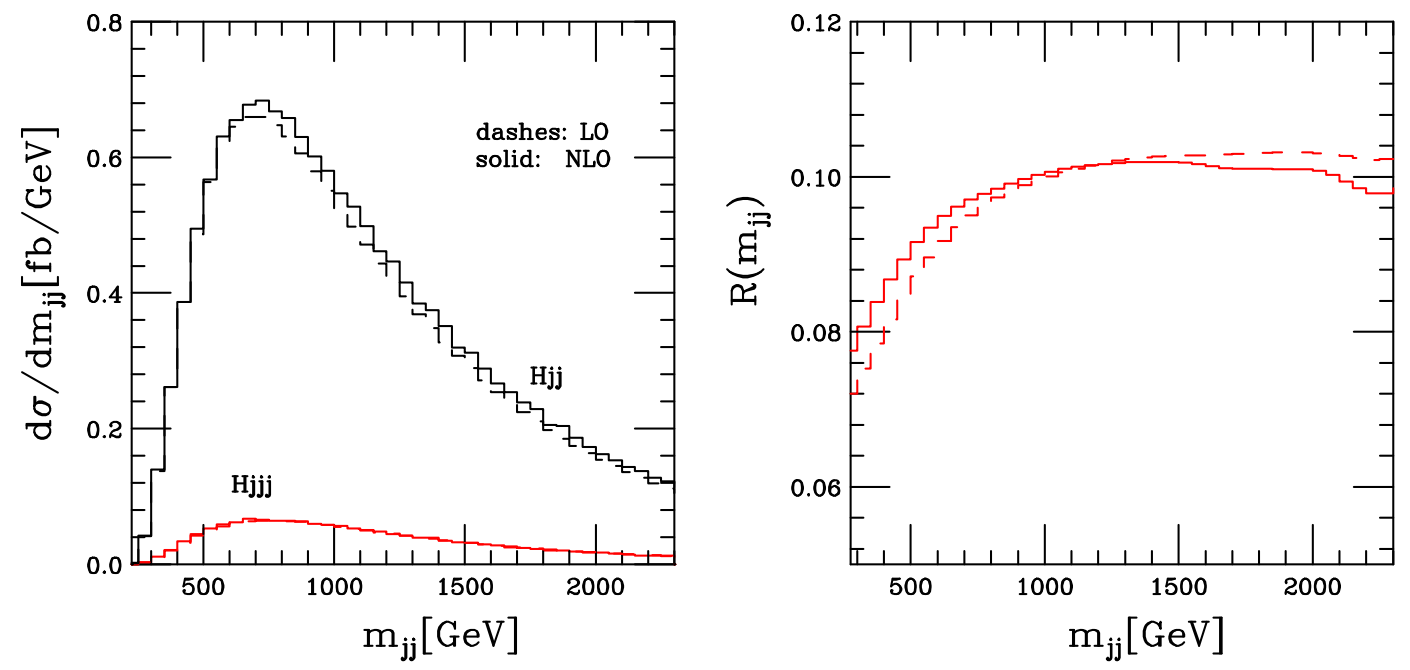

Figure 15: The invariant mass distribution of the two tagging jets for $m_{h}=120 \mathrm{GeV}$ within the cuts of Eqs. (3.1)-(3.5), Eq. (3.6) and Eq. (3.12). In the left panel, $d \sigma / d m_{j j}$ is shown at NLO (solid) and LO (dashed) for $H j j$ and for $H j j j$ production at $\mu_{R}=\mu_{F}=40 \mathrm{GeV}$. In the right panel, the corresponding 3-jet ratios, $R^{L O}\left(m_{j j}\right)$ (dashed) and $R^{N L O}\left(m_{j j}\right)$ (solid) are shown for the same scale choice.

In Fig. 17 we show the probability for finding a veto jet,

$$
P_{\text {veto }}=P\left(p_{T, \text { veto }}\right)=\frac{1}{\sigma_{2}^{N L O}} \int_{p_{T, \text { veto }}}^{\infty} d p_{T j}^{\text {veto }} \frac{d \sigma_{3}}{d p_{T j}^{\text {veto }}}
$$

as a function of the minimum transverse momentum of the hardest veto jet, $p_{T, v e t o}$. The scale variations at $\mathrm{LO}$ for the absolute veto probability are on the order of up to $\pm 3 \%$. The NLO corrections reduce this scale dependence to below the $1 \%$ level, i.e. to a negligible uncertainty. When imposing a central jet veto, the accepted VBF Higgs production cross 

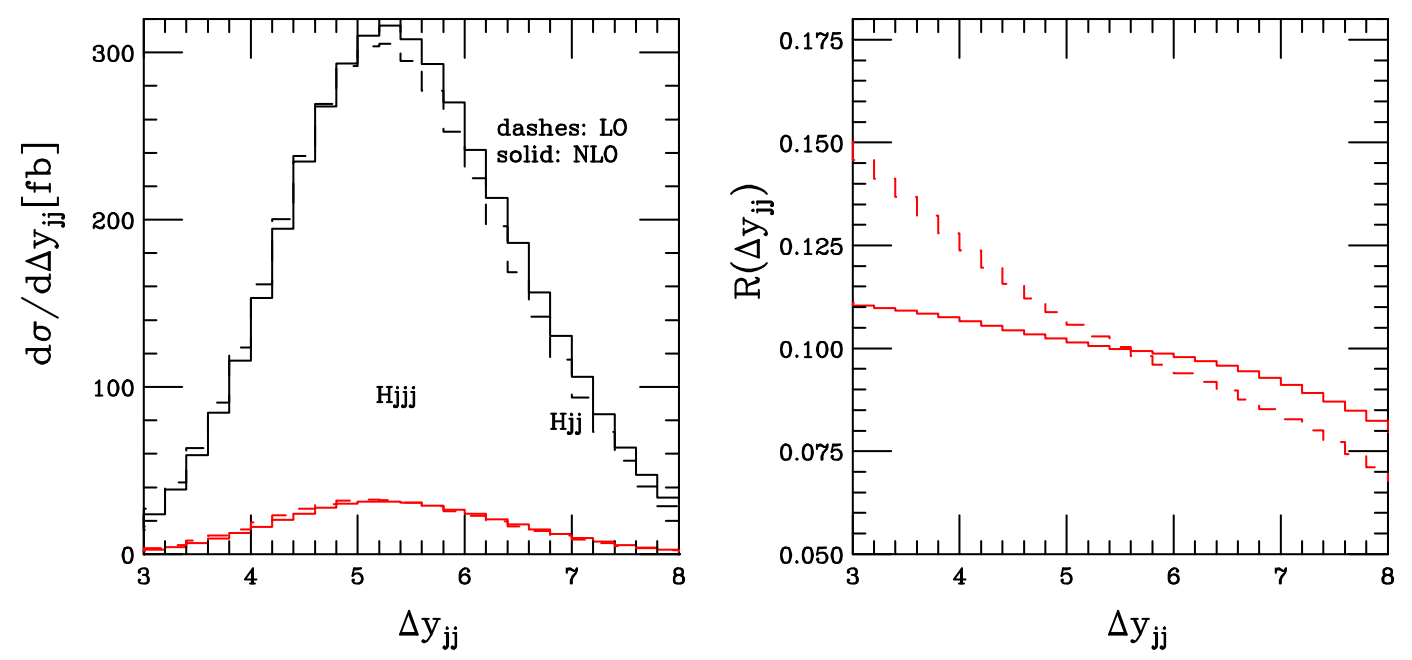

Figure 16: Same as Fig. 15 but for the rapidity separation of the two tagging jets and within the cuts of Eqs. (3.1)-(3.5), Eq. (3.7), and Eq. 3.12).

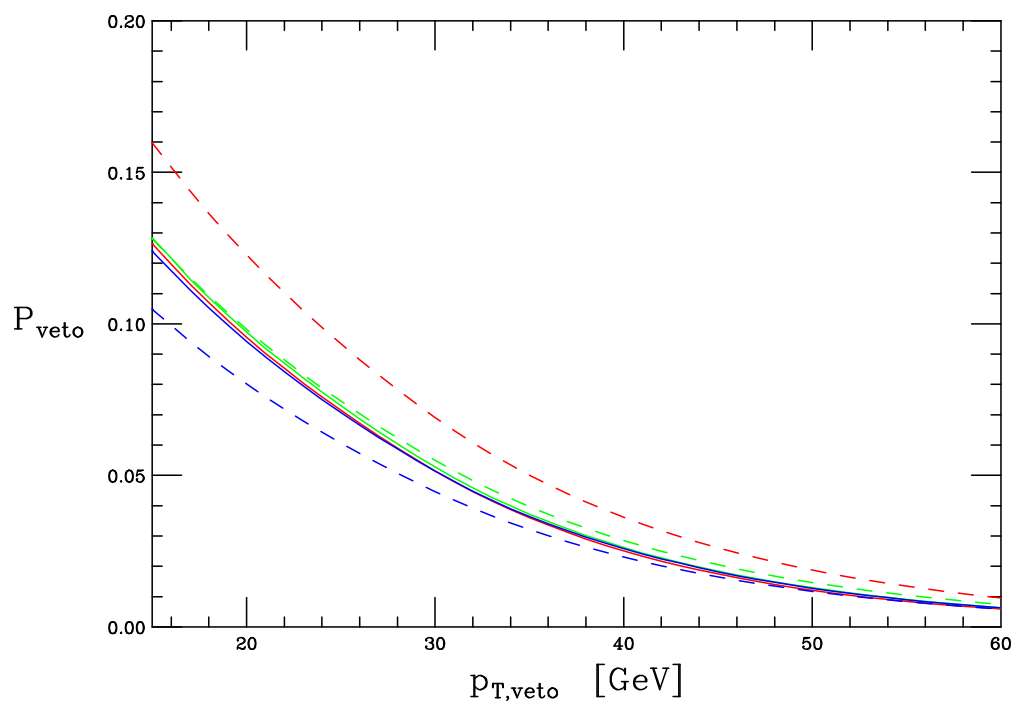

Figure 17: Ratio of the 3-jet cross section to the NLO 2-jet cross section for VBF, $P_{\text {veto }}=$ $\sigma_{3} / \sigma_{2}^{N L O}$. The dashed curves depict LO ratios and solid curves depict NLO ratios for the following scale choices: $\mu_{R}=\mu_{F}=20 \mathrm{GeV}$ (red), $\mu_{R}=\mu_{F}=40 \mathrm{GeV}$ (green), and $\mu_{R}=\mu_{F}=80 \mathrm{GeV}$ (blue).

section is given by

$$
\sigma_{2}(\text { veto })=\left(1-P_{\text {veto }}\right) \sigma_{2}
$$

Since, at NLO, $P_{\text {veto }}$ is only about $10 \%$ for a veto jet $p_{T}$ threshold of $20 \mathrm{GeV}$ (and lower for harder thresholds) the perturbative uncertainty on the SM prediction for the Higgs cross section due to a central jet veto is of order $1 \%$ only at NLO and hence negligible compared to expected statistical errors 邨. 


\section{Discussion and Conclusions}

In this paper we have presented the dominant QCD corrections for Higgs production via vector-boson fusion in association with three jets. The calculations are implemented in the form of a parton-level Monte Carlo program which allows to analyze arbitrary collinear and infrared safe distributions with NLO QCD accuracy.

Our calculation involves several approximations which significantly reduce the complexity of the virtual corrections. Since we are only interested in phase space regions where vector boson fusion processes can be distinguished from QCD backgrounds, we neglect contributions which are small once typical VBF cuts, in particular wide tagging jet separations and large tagging jet invariant masses, are imposed. Identical fermion interference effects are small after VBF cuts and we have also estimated the contribution from $t$-channel gluon exchange in virtual diagrams (and related real emission diagrams) to be well below one permille over the entire phase space relevant for VBF production. Neglecting these small contributions, the QCD 1-loop corrections involve only a single quark line and are similar in complexity to dijet production in DIS [33], i.e. they require the calculation of box diagrams as the most complex ingredient.

One reason for the smallness of the $t$-channel gluon contributions is that they are color suppressed, by a factor of $1 /\left(N^{2}-1\right)$ in an $S U(N)$ gauge theory, and this feature is generic since gluon colors need to be correlated to match the color singlet exchange nature of the tree level VBF process. In addition, we find very strong kinematical suppression factors in our analysis of the $t$-channel gluon contributions which can be traced to the characteristic gluon radiation pattern in VBF events. It is this kinematical suppression which renders the $t$-channel gluon contributions truly negligible. It would be interesting to find out, whether this kinematical suppression persists at higher orders.

In our phenomenological analysis for the LHC we find that additional jet activity between the tagging jets in VBF Higgs production events is even more strongly suppressed once NLO QCD corrections are included: $K$ factors go down to 0.7 for jet emission at the center between the two tagging jets. This strengthens the case for a central jet veto as a background suppression tool. Requiring the absence of any additional jet activity of $p_{T j}>20 \mathrm{GeV}$ between the the tagging jets we find veto probabilities for the signal of $P_{\text {veto }} \approx 10 \%$ from this perturbative QCD source. Our NLO QCD predictions for the veto probability show small residual scale variations, indicating a relative error on $P_{\text {veto }}$ due to higher order effects of $10 \%$ or less. This implies that the survival probability $P_{\text {surv }}=1-P_{\text {veto }}$ can be determined with a perturbative QCD uncertainty of about $1 \%$, which is more than sufficient for Higgs coupling determinations at the LHC 沟.

Beyond the additional jet activity from perturbative QCD radiation, which we have analyzed in this paper, additional central jets in VBF events will arise from multiple parton scattering (i.e. the underlying event) and from pile-up in high luminosity running. For

small veto thresholds $p_{T, v e t o}$, the contributions from these sources may be as large as the perturbative effects which we have considered and need to be estimated independently. However, these additional contributions should be independent of the hard scattering event and can, hence, be determined from other LHC data, in particular by measuring the jet 
activity in other VBF processes. What will be needed on the theoretical side, is a precise calculation of the perturbative contribution to the veto probability for these other VBF processes, similar to the calculation described in this paper. 


\section{Acknowledgments}

This research was supported in part by the Deutsche Forschungsgemeinschaft under SFB TR-9 "Computational Particle Physics" and via the Graduiertenkolleg "High Energy Physics and Particle Astrophysics" and in part by the European Community's Marie-Curie Research Training Network under contract MRTN-CT-2006-035505 'Tools and Precision Calculations for Physics Discoveries at Colliders'. We also thank the Galileo Galilei Institute for Theoretical Physics for the hospitality and the INFN for partial support during the completion of this work. 


\section{A. Virtual Corrections}

In this appendix, we give the expressions for the finite, reduced amplitudes, $\widetilde{\mathcal{M}}_{\tau}^{(i)}$ that appear in Eq. (2.23) in terms of $\widetilde{B}_{0}, \widetilde{C}_{0}$, and $\widetilde{D}_{i j}$ functions. Here $\widetilde{B}_{0}, \widetilde{C}_{0}$, and $\widetilde{D}_{i j}$ denote the finite parts of the Passarino-Veltman $B_{0}, C_{0}$, and $D_{i j}$ functions [28], and are given explicity below. We write

$$
\begin{aligned}
\widetilde{\mathcal{M}}_{\tau}^{(1)}\left(k_{2}, q_{1}, q_{2} ; \epsilon_{1}, \epsilon_{2}\right) & =\bar{\psi}\left(k_{2}\right)\left\{c_{q}^{(1)}\left(\phi_{1}-q_{2}\right)+c_{1}^{(1)} \phi_{1}+c_{2}^{(1)} \phi_{2}\right. \\
& \left.+c_{b}^{(1)} \phi_{2}\left(\not k_{2}+\phi_{2}\right) \phi_{1}\right\} P_{\tau} \psi\left(k_{1}\right), \\
\widetilde{\mathcal{M}}_{\tau}^{(2)}\left(k_{2}, q_{1}, q_{2} ; \epsilon_{1}, \epsilon_{2}\right) & =\bar{\psi}\left(k_{2}\right)\left\{c_{q}^{(2)}\left(\phi_{1}-\phi_{2}\right)+c_{1}^{(2)} \phi_{1}+c_{2}^{(2)} \phi_{2}\right. \\
& \left.+c_{b}^{(2)} \phi_{1}\left(\not k_{2}+\phi_{1}\right) \phi_{2}\right\} P_{\tau} \psi\left(k_{1}\right),
\end{aligned}
$$

and,

$$
\begin{aligned}
\widetilde{\mathcal{M}}_{\tau}^{(3)}\left(k_{2}, q_{1}, q_{2} ; \epsilon_{1}, \epsilon_{2}\right) & =\bar{\psi}\left(k_{2}\right)\left\{c_{q}^{(3)}\left(\phi_{1}-\phi_{2}\right)+c_{1}^{(3)} \phi_{1}+c_{2}^{(3)} \phi_{2}\right. \\
& \left.+c_{b}^{(3)}(u) \phi_{1}\left(\not k_{2}+\phi_{1}\right) \phi_{2}+c_{b}^{(3)}(t) \phi_{2}\left(\not k_{2}+q_{2}\right) \phi_{1}\right\} P_{\tau} \psi\left(k_{1}\right),
\end{aligned}
$$

where $\epsilon_{1}=\epsilon_{1}\left(q_{1}\right)$ and $\epsilon_{2}=\epsilon_{2}\left(q_{2}\right)$ are the effective polarization vectors for the gluon and weak boson. The coefficient functions, $c_{i}^{(j)}$, with $j=1,2,3$ and $i=b, q, 1,2$ are given below.

$$
\begin{aligned}
& c_{b}^{(1)}=\mathbf{B o x}_{b}^{(1)}-\frac{2 \widetilde{B}_{0}(t)}{t}-\frac{T_{b}\left(q_{2}^{2}, t\right)}{t} \\
& c_{1}^{(1)}=\operatorname{Box}_{1}^{(1)}+2 \epsilon_{2} \cdot k_{2} T_{\epsilon}\left(q_{2}^{2}, t\right)-2 \epsilon_{2} \cdot q_{2} \frac{\left[\widetilde{B}_{0}(t)-\widetilde{B}_{0}\left(q_{2}^{2}\right)\right]}{t-q_{2}^{2}} \\
& c_{2}^{(1)}=\mathbf{B o x}_{2}^{(1)}+2 \epsilon_{1} \cdot k_{1} T_{\epsilon}(0, t) \\
& c_{q}^{(1)}=\operatorname{Box}_{q}^{(1)} \\
& c_{b}^{(2)}=\mathbf{B o x}_{b}^{(2)}-\frac{2 \widetilde{B}_{0}(u)}{u}-\frac{T_{b}\left(q_{2}^{2}, u\right)}{u} \\
& c_{1}^{(2)}=\mathbf{B o x}_{1}^{(2)}+2 \epsilon_{2} \cdot k_{1} T_{\epsilon}\left(q_{2}^{2}, u\right)+2 \epsilon_{2} \cdot q_{2} \frac{\left[\widetilde{B}_{0}(u)-\widetilde{B}_{0}\left(q_{2}^{2}\right)\right]}{u-q_{2}^{2}} \\
& c_{2}^{(2)}=\mathbf{B o x}_{2}^{(2)}+2 \epsilon_{1} \cdot k_{2} T_{\epsilon}(0, u) \\
& c_{q}^{(2)}=\operatorname{Box}_{q}^{(2)} \\
& t c_{b}^{(3)}(t)=t \mathbf{B o x}_{b}^{(3)}-2\left(t \widetilde{C}_{0}(t)+1\right)+\widetilde{B}_{0}(t)+T_{b}\left(q_{2}^{2}, t\right) \\
& u c_{b}^{(3)}(u)=u \operatorname{Box}_{b}^{(3)}-2\left(u \widetilde{C}_{0}(u)+1\right)+\widetilde{B}_{0}(u)+T_{b}\left(q_{2}^{2}, u\right) \\
& c_{1}^{(3)}=\operatorname{Box}_{1}^{(3)}-2 \epsilon_{2} \cdot k_{2} T_{\epsilon}\left(q_{2}^{2}, t\right)+2 \epsilon_{2} \cdot q_{2} \frac{\left[\widetilde{B}_{0}(t)-\widetilde{B}_{0}\left(q_{2}^{2}\right)\right]}{t-q_{2}^{2}} \\
& -2 \epsilon_{2} \cdot k_{1} T_{\epsilon}\left(q_{2}^{2}, u\right)-2 \epsilon_{2} \cdot q_{2} \frac{\left[\widetilde{B}_{0}(u)-\widetilde{B}_{0}\left(q_{2}^{2}\right)\right]}{u-q_{2}^{2}} \\
& c_{2}^{(3)}=\operatorname{Box}_{2}^{(3)}+\frac{2}{t}\left(t \widetilde{C}_{0}(t)+1\right) \epsilon_{1} \cdot k_{1}+\frac{2}{u}\left(u \widetilde{C}_{0}(u)+1\right) \epsilon_{1} \cdot k_{2} \\
& c_{q}^{(3)}=\operatorname{Box}_{q}^{(3)}
\end{aligned}
$$


The $T_{i}$ functions are explicitly listed below.

$$
\begin{aligned}
T_{b}\left(q^{2}, t\right)= & \frac{1}{t-q^{2}}\left\{2 q^{2}\left[\widetilde{B}_{0}(t)-\widetilde{B}_{0}\left(q^{2}\right)\right]+t \widetilde{B}_{0}(t)\right. \\
& \left.-q^{2} \widetilde{B}_{0}\left(q^{2}\right)\right\}-2 q^{2} \widetilde{C}_{0}\left(q^{2}, t\right) \\
T_{\epsilon}\left(q^{2}, t\right)= & \frac{1}{t-q^{2}}\left\{\left[\widetilde{B}_{0}(t)-\widetilde{B}_{0}\left(q^{2}\right)\right] \frac{2 t+3 q^{2}}{t-q^{2}}+2 \widetilde{B}_{0}\left(q^{2}\right)+1-2 q^{2} \widetilde{C}_{0}\left(q^{2}, t\right)\right\} \\
T_{\epsilon}(0, t)= & \frac{1}{t}\left(2 \widetilde{B}_{0}(t)+1\right) \\
T_{b}(0, t)= & \widetilde{B}_{0}(t)
\end{aligned}
$$

Here the coefficients, Box $_{i}^{j}$, for $j=1,2,3$ and $i=b, q, 1,2$ are expressed in terms of the Passarino-Veltman $\widetilde{D}_{i j}$ functions. The Box coefficients with $\widetilde{D}_{i j}=\widetilde{D}_{i j}\left(q_{1}, k_{2}, q_{2}\right)$ are listed below.

$$
\begin{aligned}
\operatorname{Box}_{b}^{(3)} & =\left(6 \widetilde{D}_{27}+\frac{3}{2} \widetilde{D}_{0} q_{2}^{2}+\frac{5}{2} \widetilde{D}_{12} q_{2}^{2}-\widetilde{D}_{13} q_{2}^{2}-3 \widetilde{D}_{23} q_{2}^{2}+\widetilde{D}_{24} q_{2}^{2}-2 \widetilde{D}_{25} q_{2}^{2}\right. \\
& +4 \widetilde{D}_{26} q_{2}^{2}+\frac{1}{2} \widetilde{D}_{0} t+3 \widetilde{D}_{11} t-\frac{5}{2} \widetilde{D}_{12} t+\widetilde{D}_{21} t-\widetilde{D}_{24} t+4 \widetilde{D}_{25} t \\
& -4 \widetilde{D}_{26} t-\frac{3}{2} \widetilde{D}_{0} u-\widetilde{D}_{11} u-\frac{9}{2} \widetilde{D}_{12} u+4 \widetilde{D}_{13} u+\widetilde{D}_{21} u \\
& \left.-5 \widetilde{D}_{24} u+4 \widetilde{D}_{25} u\right) / 2
\end{aligned}
$$

$$
\begin{aligned}
& \operatorname{Box}_{q}^{(3)}=-\widetilde{D}_{27} \epsilon_{1} \cdot \epsilon_{2}-2 \widetilde{D}_{311} \epsilon_{1} \cdot \epsilon_{2}+2 \widetilde{D}_{313} \epsilon_{1} \cdot \epsilon_{2}-8 \widetilde{D}_{12} \epsilon_{1} \cdot k_{2} \epsilon_{2} \cdot k_{2} \\
& +8 \widetilde{D}_{13} \epsilon_{1} \cdot k_{2} \epsilon_{2} \cdot k_{2}-4 \widetilde{D}_{22} \epsilon_{1} \cdot k_{2} \epsilon_{2} \cdot k_{2}-8 \widetilde{D}_{24} \epsilon_{1} \cdot k_{2} \epsilon_{2} \cdot k_{2}+12 \widetilde{D}_{26} \epsilon_{1} \cdot k_{2} \epsilon_{2} \cdot k_{2} \\
& -4 \widetilde{D}_{36} \epsilon_{1} \cdot k_{2} \epsilon_{2} \cdot k_{2}+4 \widetilde{D}_{38} \epsilon_{1} \cdot k_{2} \epsilon_{2} \cdot k_{2}+\frac{3}{2} \widetilde{D}_{0} \epsilon_{1} \cdot q_{2} \epsilon_{2} \cdot k_{2}+\frac{3}{2} \widetilde{D}_{12} \epsilon_{1} \cdot q_{2} \epsilon_{2} \cdot k_{2} \\
& +4 \widetilde{D}_{23} \epsilon_{1} \cdot q_{2} \epsilon_{2} \cdot k_{2}-8 \widetilde{D}_{25} \epsilon_{1} \cdot q_{2} \epsilon_{2} \cdot k_{2}+4 \widetilde{D}_{26} \epsilon_{1} \cdot q_{2} \epsilon_{1} \cdot k_{2}-4 \widetilde{D}_{310} \epsilon_{1} \cdot q_{2} \epsilon_{2} \cdot k_{2} \\
& +4 \widetilde{D}_{39} \epsilon_{1} \cdot q_{2} \epsilon_{2} \cdot k_{2}-\frac{3}{2} \widetilde{D}_{0} \epsilon_{1} \cdot k_{2} \epsilon_{2} \cdot q_{1}-\frac{19}{2} \widetilde{D}_{12} \epsilon_{1} \cdot k_{2} \epsilon_{2} \cdot q_{1}+8 \widetilde{D}_{13} \epsilon_{1} \cdot k_{2} \epsilon_{2} \cdot q_{1} \\
& -12 \widetilde{D}_{24} \epsilon_{1} \cdot k_{2} \epsilon_{2} \cdot q_{1}+8 \widetilde{D}_{25} \epsilon_{1} \cdot k_{2} \epsilon_{2} \cdot q_{1}+4 \widetilde{D}_{26} \epsilon_{1} \cdot k_{2} \epsilon_{2} \cdot q_{1}+4 \widetilde{D}_{310} \epsilon_{1} \cdot k_{2} \epsilon_{2} \cdot q_{1} \\
& -4 \widetilde{D}_{34} \epsilon_{1} \cdot k_{2} \epsilon_{2} \cdot q_{1}+4 \widetilde{D}_{23} \epsilon_{1} \cdot q_{2} \epsilon_{2} \cdot q_{1}-4 \widetilde{D}_{25} \epsilon_{1} \cdot q_{2} \epsilon_{2} \cdot q_{1}-4 \widetilde{D}_{35} \epsilon_{1} \cdot q_{2} \epsilon_{2} \cdot q_{1} \\
& +4 \widetilde{D}_{37} \epsilon_{1} \cdot q_{2} \epsilon_{2} \cdot q_{1}-\frac{3}{2} \widetilde{D}_{0} \epsilon_{1} \cdot k_{2} \epsilon_{2} \cdot q_{2}-\frac{11}{2} \widetilde{D}_{12} \epsilon_{1} \cdot k_{2} \epsilon_{2} \cdot q_{2}+4 \widetilde{D}_{13} \epsilon_{1} \cdot k_{2} \epsilon_{2} \cdot q_{2} \\
& +8 \widetilde{D}_{23} \epsilon_{1} \cdot k_{2} \epsilon_{2} \cdot q_{2}-4 \widetilde{D}_{24} \epsilon_{1} \cdot k_{2} \epsilon_{2} \cdot q_{2}-4 \widetilde{D}_{26} \epsilon_{1} \cdot k_{2} \epsilon_{2} \cdot q_{2}-4 \widetilde{D}_{310} \epsilon_{1} \cdot k_{2} \epsilon_{2} \cdot q_{2} \\
& +4 \widetilde{D}_{39} \epsilon_{1} \cdot k_{2} \epsilon_{2} \cdot q_{2}+4 \widetilde{D}_{23} \epsilon_{1} \cdot q_{2} \epsilon_{2} \cdot q_{2}-4 \widetilde{D}_{25} \epsilon_{1} \cdot q_{2} \epsilon_{2} \cdot q_{2}+4 \widetilde{D}_{33} \epsilon_{1} \cdot q_{2} \epsilon_{2} \cdot q_{2} \\
& \left.-4 \widetilde{D}_{37} \epsilon_{1} \cdot q_{2} \epsilon_{2} \cdot q_{2}-\frac{1}{2} \widetilde{D}_{12} \epsilon_{1} \cdot \epsilon_{2} q_{2}^{2}+\frac{1}{2} \widetilde{D}_{13} \epsilon_{1} \cdot \epsilon_{2} q_{2}^{2}+\frac{1}{2} \widetilde{D}_{23} \epsilon_{1} \cdot \epsilon_{2} q_{2}^{2}\right) \\
& -\frac{1}{2} \widetilde{D}_{24} \epsilon_{1} \cdot \epsilon_{2} q_{2}^{2}-\widetilde{D}_{310} \epsilon_{1} \cdot \epsilon_{2} q_{2}^{2}-\widetilde{D}_{33} \epsilon_{1} \cdot \epsilon_{2} q_{2}^{2}+\widetilde{D}_{37} \epsilon_{1} \cdot \epsilon_{2} q_{2}^{2} \\
& +\widetilde{D}_{39} \epsilon_{1} \cdot \epsilon_{2} q_{2}^{2}-\widetilde{D}_{0} \epsilon_{1} \cdot \epsilon_{2} t-\frac{3}{2} \widetilde{D}_{11} \epsilon_{1} \cdot \epsilon_{2} t+\frac{1}{2} \widetilde{D}_{12} \epsilon_{1} \cdot \epsilon_{2} t \\
& -\frac{1}{2} \widetilde{D}_{21} \epsilon_{1} \cdot \epsilon_{2} t+\frac{1}{2} \widetilde{D}_{24} \epsilon_{1} \cdot \epsilon_{2} t+\widetilde{D}_{310} \epsilon_{1} \cdot \epsilon_{2} t-\widetilde{D}_{35} \epsilon_{1} \cdot \epsilon_{2} t \\
& +\widetilde{D}_{37} \epsilon_{1} \cdot \epsilon_{2} t-\widetilde{D}_{39} \epsilon_{1} \cdot \epsilon_{2} t-\frac{11}{2} \widetilde{D}_{11} \epsilon_{1} \cdot \epsilon_{2} u+\frac{3}{2} \widetilde{D}_{12} \epsilon_{1} \cdot \epsilon_{2} u
\end{aligned}
$$


- $\widetilde{D}_{13} \epsilon_{1} \cdot \epsilon_{2} u+\frac{1}{2} \widetilde{D}_{21} \epsilon_{1} \cdot \epsilon_{2} u+\widetilde{D}_{23} \epsilon_{1} \cdot \epsilon_{2} u+\frac{3}{2} \widetilde{D}_{24} \epsilon_{1} \cdot \epsilon_{2} u$

$-2 \widetilde{D}_{25} \epsilon_{1} \cdot \epsilon_{2} u-\widetilde{D}_{26} \epsilon_{1} \cdot \epsilon_{2} u-\widetilde{D}_{310} \epsilon_{1} \cdot \epsilon_{2} u+\widetilde{D}_{34} \epsilon_{1} \cdot \epsilon_{2} u$

- $\widetilde{D}_{35} \epsilon_{1} \cdot \epsilon_{2} u+\widetilde{D}_{37} \epsilon_{1} \cdot \epsilon_{2} u$

$$
\begin{aligned}
& \mathbf{B o x}_{1}^{(3)}=24 \widetilde{D}_{27} \epsilon_{2} \cdot k_{2}+20 \widetilde{D}_{312} \epsilon_{2} \cdot k_{2}+22 \widetilde{D}_{27} \epsilon_{2} \cdot q_{1}+20 \widetilde{D}_{311} \epsilon_{2} \cdot q_{1} \\
& +12 \widetilde{D}_{27} \epsilon_{2} \cdot q_{2}+20 \widetilde{D}_{313} \epsilon_{2} \cdot q_{2}-4 \widetilde{D}_{23} \epsilon_{2} \cdot k_{2} q_{2}^{2}+4 \widetilde{D}_{26} \epsilon_{2} \cdot k_{2} q_{2}^{2} \\
& +2 \widetilde{D}_{38} \epsilon_{2} \cdot k_{2} q_{2}^{2}-2 \widetilde{D}_{39} \epsilon_{2} \cdot k_{2} q_{2}^{2}+\widetilde{D}_{12} \epsilon_{2} \cdot q_{1} q_{2}^{2}-\widetilde{D}_{13} \epsilon_{2} \cdot q_{1} q_{2}^{2} \\
& -3 \widetilde{D}_{23} \epsilon_{2} \cdot q_{1} q_{2}^{2}+\widetilde{D}_{24} \epsilon_{2} \cdot q_{1} q_{2}^{2}+2 \widetilde{D}_{26} \epsilon_{2} \cdot q_{1} q_{2}^{2}+2 \widetilde{D}_{310} \epsilon_{2} \cdot q_{1} q_{2}^{2} \\
& -2 \widetilde{D}_{37} \epsilon_{2} \cdot q_{1} q_{2}^{2}-2 \widetilde{D}_{23} \epsilon_{2} \cdot q_{2} q_{2}^{2}+2 \widetilde{D}_{26} \epsilon_{2} \cdot q_{2} q_{2}^{2}-2 \widetilde{D}_{33} \epsilon_{2} \cdot q_{2} q_{2}^{2} \\
& +2 \widetilde{D}_{39} \epsilon_{2} \cdot q_{2} q_{2}^{2}+\frac{3}{2} \widetilde{D}_{0} \epsilon_{2} \cdot k_{2} t+\frac{3}{2} \widetilde{D}_{12} \epsilon_{2} \cdot k_{2} t+4 \widetilde{D}_{13} \epsilon_{2} \cdot k_{2} t \\
& +2 \widetilde{D}_{25} \epsilon_{2} \cdot k_{2} t+2 \widetilde{D}_{26} \epsilon_{2} \cdot k_{2} t+2 \widetilde{D}_{310} \epsilon_{2} \cdot k_{2} t-2 \widetilde{D}_{38} \epsilon_{2} \cdot k_{2} t \\
& +2 \widetilde{D}_{0} \epsilon_{2} \cdot q_{1} t+3 \widetilde{D}_{11} \epsilon_{2} \cdot q_{1} t-\widetilde{D}_{12} \epsilon_{2} \cdot q_{1} t+4 \widetilde{D}_{13} \epsilon_{2} \cdot q_{1} t \\
& +\widetilde{D}_{21} \epsilon_{2} \cdot q_{1} t-\widetilde{D}_{24} \epsilon_{2} \cdot q_{1} t+6 \widetilde{D}_{25} \epsilon_{2} \cdot q_{1} t-2 \widetilde{D}_{26} \epsilon_{2} \cdot q_{1} t \\
& -2 \widetilde{D}_{310} \epsilon_{2} \cdot q_{1} t+2 \widetilde{D}_{35} \epsilon_{2} \cdot q_{1} t+4 \widetilde{D}_{13} \epsilon_{2} \cdot q_{2} t+4 \widetilde{D}_{23} \epsilon_{2} \cdot q_{2} t \\
& +2 \widetilde{D}_{25} \epsilon_{2} \cdot q_{2} t-2 \widetilde{D}_{26} \epsilon_{2} \cdot q_{2} t+2 \widetilde{D}_{37} \epsilon_{2} \cdot q_{2} t-2 \widetilde{D}_{39} \epsilon_{2} \cdot q_{2} t \\
& +\frac{3}{2} \widetilde{D}_{0} \epsilon_{2} \cdot k_{2} u+\frac{7}{2} \widetilde{D}_{12} \epsilon_{2} \cdot k_{2} u-2 \widetilde{D}_{13} \epsilon_{2} \cdot k_{2} u+2 \widetilde{D}_{22} \epsilon_{2} \cdot k_{2} u \\
& -2 \widetilde{D}_{24} \epsilon_{2} \cdot k_{2} u+2 \widetilde{D}_{25} \epsilon_{2} \cdot k_{2} u-2 \widetilde{D}_{26} \epsilon_{2} \cdot k_{2} u+2 \widetilde{D}_{310} \epsilon_{2} \cdot k_{2} u \\
& -2 \widetilde{D}_{36} \epsilon_{2} \cdot k_{2} u+\frac{3}{2} \widetilde{D}_{0} \epsilon_{2} \cdot q_{1} u+\widetilde{D}_{11} \epsilon_{2} \cdot q_{1} u+\frac{5}{2} \widetilde{D}_{12} \epsilon_{2} \cdot q_{1} u \\
& -2 \widetilde{D}_{13} \epsilon_{2} \cdot q_{1} u-\widetilde{D}_{21} \epsilon_{2} \cdot q_{1} u+\widetilde{D}_{24} \epsilon_{2} \cdot q_{1} u-2 \widetilde{D}_{34} \epsilon_{2} \cdot q_{1} u \\
& +2 \widetilde{D}_{35} \epsilon_{2} \cdot q_{1} u+\frac{3}{2} \widetilde{D}_{0} \epsilon_{2} \cdot q_{2} u+\frac{3}{2} \widetilde{D}_{12} \epsilon_{2} \cdot q_{2} u-2 \widetilde{D}_{23} \epsilon_{2} \cdot q_{2} u \\
& +2 \widetilde{D}_{26} \epsilon_{2} \cdot q_{2} u-2 \widetilde{D}_{310} \epsilon_{2} \cdot q_{2} u+2 \widetilde{D}_{37} \epsilon_{2} \cdot q_{2} u
\end{aligned}
$$

$$
\begin{aligned}
\mathbf{B o x}_{2}^{(3)} & =-12 \widetilde{D}_{27} \epsilon_{1} \cdot k_{2}-4 \widetilde{D}_{312} \epsilon_{1} \cdot k_{2}-6 \widetilde{D}_{27} \epsilon_{1} \cdot q_{2}-4 \widetilde{D}_{313} \epsilon_{1} \cdot q_{2} \\
& -3 \widetilde{D}_{0} \epsilon_{1} \cdot k_{2} q_{2}^{2}-7 \widetilde{D}_{12} \epsilon_{1} \cdot k_{2} q_{2}^{2}+2 \widetilde{D}_{13} \epsilon_{1} \cdot k_{2} q_{2}^{2}-2 \widetilde{D}_{22} \epsilon_{1} \cdot k_{2} q_{2}^{2} \\
& +6 \widetilde{D}_{23} \epsilon_{1} \cdot k_{2} q_{2}^{2}-2 \widetilde{D}_{24} \epsilon_{1} \cdot k_{2} q_{2}^{2}+4 \widetilde{D}_{25} \epsilon_{1} \cdot k_{2} q_{2}^{2}-8 \widetilde{D}_{26} \epsilon_{1} \cdot k_{2} q_{2}^{2} \\
& -2 \widetilde{D}_{38} \epsilon_{1} \cdot k_{2} q_{2}^{2}+2 \widetilde{D}_{39} \epsilon_{1} \cdot k_{2} q_{2}^{2}-\frac{3}{2} \widetilde{D}_{0} \epsilon_{1} \cdot q_{2} q_{2}^{2}-\frac{5}{2} \widetilde{D}_{12} \epsilon_{1} \cdot q_{2} q_{2}^{2} \\
& -\widetilde{D}_{13} \epsilon_{1} \cdot q_{2} q_{2}^{2}+3 \widetilde{D}_{23} \epsilon_{1} \cdot q_{2} q_{2}^{2}-\widetilde{D}_{24} \epsilon_{1} \cdot q_{2} q_{2}^{2}+2 \widetilde{D}_{25} \epsilon_{1} \cdot q_{2} q_{2}^{2} \\
& -6 \widetilde{D}_{26} \epsilon_{1} \cdot q_{2} q_{2}^{2}+2 \widetilde{D}_{33} \epsilon_{1} \cdot q_{2} q_{2}^{2}-2 \widetilde{D}_{39} \epsilon_{1} \cdot q_{2} q_{2}^{2}+\frac{3}{2} \widetilde{D}_{0} \epsilon_{1} \cdot k_{2} t \\
& -4 \widetilde{D}_{11} \epsilon_{1} \cdot k_{2} t+\frac{11}{2} \widetilde{D}_{12} \epsilon_{1} \cdot k_{2} t-2 \widetilde{D}_{21} \epsilon_{1} \cdot k_{2} t+2 \widetilde{D}_{22} \epsilon_{1} \cdot k_{2} t \\
& -6 \widetilde{D}_{25} \epsilon_{1} \cdot k_{2} t+6 \widetilde{D}_{26} \epsilon_{1} \cdot k_{2} t-2 \widetilde{D}_{310} \epsilon_{1} \cdot k_{2} t+2 \widetilde{D}_{38} \epsilon_{1} \cdot k_{2} t \\
& -\frac{1}{2} \widetilde{D}_{0} \epsilon_{1} \cdot q_{2} t-3 \widetilde{D}_{11} \epsilon_{1} \cdot q_{2} t+\frac{5}{2} \widetilde{D}_{12} \epsilon_{1} \cdot q_{2} t-\widetilde{D}_{21} \epsilon_{1} \cdot q_{2} t \\
& +\widetilde{D}_{24} \epsilon_{1} \cdot q_{2} t-6 \widetilde{D}_{25} \epsilon_{1} \cdot q_{2} t+6 \widetilde{D}_{26} \epsilon_{1} \cdot q_{2} t-2 \widetilde{D}_{37} \epsilon_{1} \cdot q_{2} t
\end{aligned}
$$




$$
\begin{aligned}
& +2 \widetilde{D}_{39} \epsilon_{1} \cdot q_{2} t+\frac{7}{2} \widetilde{D}_{0} \epsilon_{1} \cdot k_{2} u+\frac{23}{2} \widetilde{D}_{12} \epsilon_{1} \cdot k_{2} u-6 \widetilde{D}_{13} \epsilon_{1} \cdot k_{2} u \\
& -2 \widetilde{D}_{21} \epsilon_{1} \cdot k_{2} u+2 \widetilde{D}_{22} \epsilon_{1} \cdot k_{2} u+10 \widetilde{D}_{24} \epsilon_{1} \cdot k_{2} u-6 \widetilde{D}_{25} \epsilon_{1} \cdot k_{2} u \\
& -2 \widetilde{D}_{26} \epsilon_{1} \cdot k_{2} u-2 \widetilde{D}_{310} \epsilon_{1} \cdot k_{2} u+2 \widetilde{D}_{36} \epsilon_{1} \cdot k_{2} u+2 \widetilde{D}_{0} \epsilon_{1} \cdot q_{2} u \\
& -\widetilde{D}_{11} \epsilon_{1} \cdot q_{2} u+5 \widetilde{D}_{12} \epsilon_{1} \cdot q_{2} u-\widetilde{D}_{21} \epsilon_{1} \cdot q_{2} u-2 \widetilde{D}_{23} \epsilon_{1} \cdot q_{2} u \\
& +3 \widetilde{D}_{24} \epsilon_{1} \cdot q_{2} u+2 \widetilde{D}_{26} \epsilon_{1} \cdot q_{2} u+2 \widetilde{D}_{310} \epsilon_{1} \cdot q_{2} u-2 \widetilde{D}_{37} \epsilon_{1} \cdot q_{2} u
\end{aligned}
$$

The Box coefficients with $\widetilde{D}_{i j}=\widetilde{D}_{i j}\left(k_{2}, q_{2}, q_{1}\right)$ are listed below.

$$
\begin{aligned}
\operatorname{Box}_{1}^{(1)} & =-\epsilon_{1} \cdot q_{1}\left[-8 \widetilde{D}_{27}-8 \widetilde{D}_{312}-\left(\widetilde{D}_{11}-\widetilde{D}_{12}+\widetilde{D}_{13}-4 \widetilde{D}_{22}+4 \widetilde{D}_{24}\right) q_{2}^{2}+\widetilde{D}_{11} s-\widetilde{D}_{12} s\right. \\
& \left.+\widetilde{D}_{13} s-4 \widetilde{D}_{22} s+4 \widetilde{D}_{24} s+\widetilde{D}_{11} t-\widetilde{D}_{12} t+\widetilde{D}_{13} t-4 \widetilde{D}_{22} t+4 \widetilde{D}_{24} t\right] \\
& +\epsilon_{2} \cdot q_{1}\left[8 \widetilde{D}_{27}+8 \widetilde{D}_{313}+\left(\widetilde{D}_{11}-\widetilde{D}_{12}+\widetilde{D}_{13}-4 \widetilde{D}_{22}+4 \widetilde{D}_{24}\right) q_{2}^{2}-\widetilde{D}_{11} t-3 \widetilde{D}_{12} t\right. \\
& \left.+3 \widetilde{D}_{13} t-4 \widetilde{D}_{24} t+4 \widetilde{D}_{26} t\right]-\epsilon_{2} \cdot k_{2}\left[16 \widetilde{D}_{311}-24 \widetilde{D}_{312}-\left(\widetilde{D}_{11}-\widetilde{D}_{12}+\widetilde{D}_{13}+4 \widetilde{D}_{25}\right.\right. \\
& \left.-4 \widetilde{D}_{26}-8 \widetilde{D}_{310}-4 \widetilde{D}_{32}-4 \widetilde{D}_{34}+4 \widetilde{D}_{35}+8 \widetilde{D}_{36}+4 \widetilde{D}_{38}\right) q_{2}^{2}+5 \widetilde{D}_{11} s-5 \widetilde{D}_{12} s \\
& +\widetilde{D}_{13} s+4 \widetilde{D}_{21} s-4 \widetilde{D}_{24} s+4 \widetilde{D}_{25} s-4 \widetilde{D}_{26} s-8 \widetilde{D}_{310} s+4 \widetilde{D}_{35} s+4 \widetilde{D}_{38} s-4 \widetilde{D}_{12} t \\
& +4 \widetilde{D}_{13} t+4 \widetilde{D}_{22} t-8 \widetilde{D}_{24} t+8 \widetilde{D}_{25} t-4 \widetilde{D}_{26} t-4 \widetilde{D}_{310} t-4 \widetilde{D}_{34} t \\
& \left.+4 \widetilde{D}_{35} t+4 \widetilde{D}_{36} t\right]
\end{aligned}
$$

$$
\begin{aligned}
\mathbf{B o x}_{2}^{(1)} & =\epsilon_{1} \cdot k_{2}\left[8 \widetilde{D}_{311}-24 \widetilde{D}_{313}-\left(\widetilde{D}_{11}+3 \widetilde{D}_{12}-3 \widetilde{D}_{13}-4 \widetilde{D}_{22}+8 \widetilde{D}_{24}-4 \widetilde{D}_{25}+4 \widetilde{D}_{310}\right.\right. \\
& \left.-4 \widetilde{D}_{37}-4 \widetilde{D}_{38}+4 \widetilde{D}_{39}\right) q_{2}^{2}-\widetilde{D}_{11} s+\widetilde{D}_{12} s-5 \widetilde{D}_{13} s-8 \widetilde{D}_{25} s+4 \widetilde{D}_{26} s-4 \widetilde{D}_{37} s \\
& \left.+4 \widetilde{D}_{39} s+4 \widetilde{D}_{12} t-4 \widetilde{D}_{13} t-4 \widetilde{D}_{23} t+4 \widetilde{D}_{24} t-4 \widetilde{D}_{25} t+4 \widetilde{D}_{26} t+4 \widetilde{D}_{310} t-4 \widetilde{D}_{37} t\right] \\
& -\epsilon_{1} \cdot q_{2}\left[8 \widetilde{D}_{27}-8 \widetilde{D}_{312}+24 \widetilde{D}_{313}+\left(\widetilde{D}_{11}+3 \widetilde{D}_{12}-3 \widetilde{D}_{13}-4 \widetilde{D}_{22}+8 \widetilde{D}_{24}-4 \widetilde{D}_{25}\right.\right. \\
& \left.+4 \widetilde{D}_{310}-4 \widetilde{D}_{37}-4 \widetilde{D}_{38}+4 \widetilde{D}_{39}\right) q_{2}^{2}+\widetilde{D}_{11} s-\widetilde{D}_{12} s+5 \widetilde{D}_{13} s+8 \widetilde{D}_{25} s-4 \widetilde{D}_{26} s \\
& +4 \widetilde{D}_{37} s-4 \widetilde{D}_{39} s-\widetilde{D}_{11} t-7 \widetilde{D}_{12} t+7 \widetilde{D}_{13} t+4 \widetilde{D}_{23} t-8 \widetilde{D}_{24} t+4 \widetilde{D}_{25} t-4 \widetilde{D}_{310} t \\
& \left.+4 \widetilde{D}_{37} t\right]
\end{aligned}
$$

$$
\begin{aligned}
& \operatorname{Box}_{q}^{(1)}=8 \widetilde{D}_{312} \epsilon_{1} \cdot \epsilon_{2}-8 \widetilde{D}_{313} \epsilon_{1} \cdot \epsilon_{2}+8 \widetilde{D}_{12} \epsilon_{1} \cdot k_{2} \epsilon_{2} \cdot k_{2}-8 \widetilde{D}_{13} \epsilon_{1} \cdot k_{2} \epsilon_{2} \cdot k_{2} \\
& +12 \widetilde{D}_{24} \epsilon_{1} \cdot k_{2} \epsilon_{2} \cdot k_{2}-12 \widetilde{D}_{25} \epsilon_{1} \cdot k_{2} \epsilon_{2} \cdot k_{2}+4 \widetilde{D}_{34} \epsilon_{1} \cdot k_{2} \epsilon_{2} \cdot k_{2}-4 \widetilde{D}_{35} \epsilon_{1} \cdot k_{2} \epsilon_{2} \cdot k_{2} \\
& +\widetilde{D}_{11} \epsilon_{1} \cdot q_{2} \epsilon_{2} \cdot k_{2}+7 \widetilde{D}_{12} \epsilon_{1} \cdot q_{2} \epsilon_{2} \cdot k_{2}-7 \widetilde{D}_{13} \epsilon_{1} \cdot q_{2} \epsilon_{2} \cdot k_{2}+4 \widetilde{D}_{22} \epsilon_{1} \cdot q_{2} \epsilon_{2} \cdot k_{2} \\
& +8 \widetilde{D}_{24} \epsilon_{1} \cdot q_{2} \epsilon_{2} \cdot k_{2}-4 \widetilde{D}_{25} \epsilon_{1} \cdot q_{2} \epsilon_{2} \cdot k_{2}-8 \widetilde{D}_{26} \epsilon_{1} \cdot q_{2} \epsilon_{2} \cdot k_{2}-4 \widetilde{D}_{310} \epsilon_{1} \cdot q_{2} \epsilon_{2} \cdot k_{2} \\
& +4 \widetilde{D}_{36} \epsilon_{1} \cdot q_{2} \epsilon_{2} \cdot k_{2}-\widetilde{D}_{11} \epsilon_{1} \cdot k_{2} \epsilon_{2} \cdot q_{1}+\widetilde{D}_{12} \epsilon_{1} \cdot k_{2} \epsilon_{2} \cdot q_{1}-\widetilde{D}_{13} \epsilon_{1} \cdot k_{2} \epsilon_{2} \cdot q_{1} \\
& -4 \widetilde{D}_{23} \epsilon_{1} \cdot k_{2} \epsilon_{2} \cdot q_{1}-4 \widetilde{D}_{25} \epsilon_{1} \cdot k_{2} \epsilon_{2} \cdot q_{1}+8 \widetilde{D}_{26} \epsilon_{1} \cdot k_{2} \epsilon_{2} \cdot q_{1}+4 \widetilde{D}_{310} \epsilon_{1} \cdot k_{2} \epsilon_{2} \cdot q_{1} \\
& -4 \widetilde{D}_{37} \epsilon_{1} \cdot k_{2} \epsilon_{2} \cdot q_{1}-4 \widetilde{D}_{23} \epsilon_{1} \cdot q_{2} \epsilon_{2} \cdot q_{1}+4 \widetilde{D}_{26} \epsilon_{1} \cdot q_{2} \epsilon_{2} \cdot q_{1}+4 \widetilde{D}_{38} \epsilon_{1} \cdot q_{2} \epsilon_{2} \cdot q_{1} \\
& -4 \widetilde{D}_{39} \epsilon_{1} \cdot q_{2} \epsilon_{2} \cdot q_{1}-\widetilde{D}_{11} \epsilon_{1} \cdot k_{2} \epsilon_{1} \cdot q_{1}+5 \widetilde{D}_{12} \epsilon_{1} \cdot k_{2} \epsilon_{1} \cdot q_{1}-5 \widetilde{D}_{13} \epsilon_{1} \cdot k_{2} \epsilon_{1} \cdot q_{1} \\
& +8 \widetilde{D}_{22} \epsilon_{1} \cdot k_{2} \epsilon_{1} \cdot q_{1}-4 \widetilde{D}_{25} \epsilon_{1} \cdot k_{2} \epsilon_{1} \cdot q_{1}-4 \widetilde{D}_{26} \epsilon_{1} \cdot k_{2} \epsilon_{1} \cdot q_{1}-4 \widetilde{D}_{310} \epsilon_{1} \cdot k_{2} \epsilon_{1} \cdot q_{1} \\
& +4 \widetilde{D}_{36} \epsilon_{1} \cdot k_{2} \epsilon_{1} \cdot q_{1}+4 \widetilde{D}_{12} \epsilon_{1} \cdot q_{2} \epsilon_{1} \cdot q_{1}-4 \widetilde{D}_{13} \epsilon_{1} \cdot q_{2} \epsilon_{1} \cdot q_{1}+8 \widetilde{D}_{22} \epsilon_{1} \cdot q_{2} \epsilon_{1} \cdot q_{1} \\
& -8 \widetilde{D}_{26} \epsilon_{1} \cdot q_{2} \epsilon_{1} \cdot q_{1}+4 \widetilde{D}_{32} \epsilon_{1} \cdot q_{2} \epsilon_{1} \cdot q_{1}-4 \widetilde{D}_{38} \epsilon_{1} \cdot q_{2} \epsilon_{1} \cdot q_{1}-4 \widetilde{D}_{310} \epsilon_{1} \cdot \epsilon_{2} q_{2}^{2} \\
& -2 \widetilde{D}_{32} \epsilon_{1} \cdot \epsilon_{2} q_{2}^{2}+2 \widetilde{D}_{36} \epsilon_{1} \cdot \epsilon_{2} q_{2}^{2}+2 \widetilde{D}_{37} \epsilon_{1} \cdot \epsilon_{2} q_{2}^{2}+4 \widetilde{D}_{38} \epsilon_{1} \cdot \epsilon_{2} q_{2}^{2}
\end{aligned}
$$


$-2 \widetilde{D}_{39} \epsilon_{1} \cdot \epsilon_{2} q_{2}^{2}-\frac{1}{2} \widetilde{D}_{11} \epsilon_{1} \cdot \epsilon_{2} s+\frac{1}{2} \widetilde{D}_{12} \epsilon_{1} \cdot \epsilon_{2} s-\frac{1}{2} \widetilde{D}_{13} \epsilon_{1} \cdot \epsilon_{2} s$

$-2 \widetilde{D}_{25} \epsilon_{1} \cdot \epsilon_{2} s+2 \widetilde{D}_{26} \epsilon_{1} \cdot \epsilon_{2} s+2 \widetilde{D}_{310} \epsilon_{1} \cdot \epsilon_{2} s-2 \widetilde{D}_{37} \epsilon_{1} \cdot \epsilon_{2} s$

$-2 \widetilde{D}_{38} \epsilon_{1} \cdot \epsilon_{2} s+2 \widetilde{D}_{39} \epsilon_{1} \cdot \epsilon_{2} s-2 \widetilde{D}_{22} \epsilon_{1} \cdot \epsilon_{2} t-2 \widetilde{D}_{23} \epsilon_{1} \cdot \epsilon_{2} t$

$+4 \widetilde{D}_{26} \epsilon_{1} \cdot \epsilon_{2} t+4 \widetilde{D}_{310} \epsilon_{1} \cdot \epsilon_{2} t-2 \widetilde{D}_{36} \epsilon_{1} \cdot \epsilon_{2} t-2 \widetilde{D}_{37} \epsilon_{1} \cdot \epsilon_{2} t$

$$
\begin{aligned}
\operatorname{Box}_{b}^{(1)} & =-4 \widetilde{D}_{27}-12 \widetilde{D}_{312}+12 \widetilde{D}_{313}+4 \widetilde{D}_{310} q_{2}^{2}+2 \widetilde{D}_{32} q_{2}^{2}-2 \widetilde{D}_{36} q_{2}^{2}-2 \widetilde{D}_{37} q_{2}^{2}-4 \widetilde{D}_{38} q_{2}^{2}+2 \widetilde{D}_{39} q_{2}^{2} \\
& -2 \widetilde{D}_{0} s-\widetilde{D}_{11} s-\widetilde{D}_{12} s+\widetilde{D}_{13} s+2 \widetilde{D}_{25} s-2 \widetilde{D}_{26} s-2 \widetilde{D}_{310} s+2 \widetilde{D}_{37} s+2 \widetilde{D}_{38} s \\
& -2 \widetilde{D}_{39} s+2 \widetilde{D}_{22} t+2 \widetilde{D}_{23} t-4 \widetilde{D}_{26} t-4 \widetilde{D}_{310} t+2 \widetilde{D}_{36} t+2 \widetilde{D}_{37} t
\end{aligned}
$$

The Box coefficients with $\widetilde{D}_{i j}=\widetilde{D}_{i j}\left(k_{2}, q_{1}, q_{2}\right)$ are listed below.

$$
\begin{aligned}
\text { Box }_{1}^{(2)} & =-\epsilon_{2} \cdot q_{1}\left[8 \widetilde{D}_{27}-8 \widetilde{D}_{312}+24 \widetilde{D}_{313}-4\left(\widetilde{D}_{23}-\widetilde{D}_{26}+\widetilde{D}_{33}-\widetilde{D}_{39}\right) q_{2}^{2}+\widetilde{D}_{11} s-\widetilde{D}_{12} s\right. \\
& +5 \widetilde{D}_{13} s+8 \widetilde{D}_{25} s-4 \widetilde{D}_{26} s+4 \widetilde{D}_{37} s-4 \widetilde{D}_{39} s-\widetilde{D}_{11} u-7 \widetilde{D}_{12} u+7 \widetilde{D}_{13} u+4 \widetilde{D}_{23} u \\
& \left.-8 \widetilde{D}_{24} u+4 \widetilde{D}_{25} u-4 \widetilde{D}_{310} u+4 \widetilde{D}_{37} u\right]-\epsilon_{2} \cdot q_{2}\left[8 \widetilde{D}_{27}+16 \widetilde{D}_{313}-4\left(\widetilde{D}_{23}-\widetilde{D}_{26}+\widetilde{D}_{33}\right.\right. \\
& \left.-\widetilde{D}_{39}\right) q_{2}^{2}+4 \widetilde{D}_{13} s+4 \widetilde{D}_{23} s+4 \widetilde{D}_{25} s-4 \widetilde{D}_{26} s+4 \widetilde{D}_{37} s-4 \widetilde{D}_{39} s-\widetilde{D}_{11} u-3 \widetilde{D}_{12} u \\
& \left.+3 \widetilde{D}_{13} u+8 \widetilde{D}_{23} u-4 \widetilde{D}_{24} u-4 \widetilde{D}_{26} u-4 \widetilde{D}_{310} u+4 \widetilde{D}_{37} u\right]-\epsilon_{2} \cdot k_{2}\left[-8 \widetilde{D}_{311}+24 \widetilde{D}_{313}\right. \\
& -\left(\widetilde{D}_{11}-\widetilde{D}_{12}+\widetilde{D}_{13}+4 \widetilde{D}_{25}-4 \widetilde{D}_{26}+4 \widetilde{D}_{33}-4 \widetilde{D}_{39}\right) q_{2}^{2}+\widetilde{D}_{11} s-\widetilde{D}_{12} s+5 \widetilde{D}_{13} s \\
& +8 \widetilde{D}_{25} s-4 \widetilde{D}_{26} s+4 \widetilde{D}_{37} s-4 \widetilde{D}_{39} s-4 \widetilde{D}_{12} u+4 \widetilde{D}_{13} u+4 \widetilde{D}_{23} u-4 \widetilde{D}_{24} u+4 \widetilde{D}_{25} u \\
& \left.-4 \widetilde{D}_{26} u-4 \widetilde{D}_{310} u+4 \widetilde{D}_{37} u\right]
\end{aligned}
$$

$$
\begin{aligned}
\operatorname{Box}_{2}^{(2)} & =-\epsilon_{1} \cdot q_{2}\left[-8 \widetilde{D}_{27}-8 \widetilde{D}_{313}+\left(\widetilde{D}_{11}+3 \widetilde{D}_{12}-3 \widetilde{D}_{13}+4 \widetilde{D}_{24}-4 \widetilde{D}_{26}\right) u\right] \\
& -\epsilon_{1} \cdot k_{2}\left[16 \widetilde{D}_{311}-24 \widetilde{D}_{312}+\left(\widetilde{D}_{11}+3 \widetilde{D}_{12}-3 \widetilde{D}_{13}-4 \widetilde{D}_{23}+4 \widetilde{D}_{24}+4 \widetilde{D}_{310}\right.\right. \\
& \left.-4 \widetilde{D}_{37}-4 \widetilde{D}_{38}+4 \widetilde{D}_{39}\right) q_{2}^{2}+5 \widetilde{D}_{11} s-5 \widetilde{D}_{12} s+\widetilde{D}_{13} s+4 \widetilde{D}_{21} s-4 \widetilde{D}_{24} s+4 \widetilde{D}_{25} s \\
& -4 \widetilde{D}_{26} s-8 \widetilde{D}_{310} s+4 \widetilde{D}_{35} s+4 \widetilde{D}_{38} s-4 \widetilde{D}_{12} u+4 \widetilde{D}_{13} u+4 \widetilde{D}_{22} u-8 \widetilde{D}_{24} u+8 \widetilde{D}_{25} u \\
& \left.-4 \widetilde{D}_{26} u-4 \widetilde{D}_{310} u-4 \widetilde{D}_{34} u+4 \widetilde{D}_{35} u+4 \widetilde{D}_{36} u\right]
\end{aligned}
$$

$$
\begin{aligned}
\operatorname{Box}_{b}^{(2)} & =-4 \widetilde{D}_{27}-12 \widetilde{D}_{312}+12 \widetilde{D}_{313}-2 \widetilde{D}_{33} q_{2}^{2}-2 \widetilde{D}_{38} q_{2}^{2}+4 \widetilde{D}_{39} q_{2}^{2}-2 \widetilde{D}_{0} s-\widetilde{D}_{11} s-\widetilde{D}_{12} s \\
& +\widetilde{D}_{13} s+2 \widetilde{D}_{25} s-2 \widetilde{D}_{26} s-2 \widetilde{D}_{310} s+2 \widetilde{D}_{37} s+2 \widetilde{D}_{38} s-2 \widetilde{D}_{39} s+2 \widetilde{D}_{22} u+2 \widetilde{D}_{23} u \\
& -4 \widetilde{D}_{26} u-4 \widetilde{D}_{310} u+2 \widetilde{D}_{36} u+2 \widetilde{D}_{37} u
\end{aligned}
$$

$$
\begin{aligned}
\mathbf{B o x}_{q}^{(2)} & =-8 \widetilde{D}_{312} \epsilon_{1} \cdot \epsilon_{2}+8 \widetilde{D}_{313} \epsilon_{1} \cdot \epsilon_{2}-8 \widetilde{D}_{12} \epsilon_{1} \cdot k_{2} \epsilon_{2} \cdot k_{2}+8 \widetilde{D}_{13} \epsilon_{1} \cdot k_{2} \epsilon_{2} \cdot k_{2} \\
& -12 \widetilde{D}_{24} \epsilon_{1} \cdot k_{2} \epsilon_{2} \cdot k_{2}+12 \widetilde{D}_{25} \epsilon_{1} \cdot k_{2} \epsilon_{2} \cdot k_{2}-4 \widetilde{D}_{34} \epsilon_{1} \cdot k_{2} \epsilon_{2} \cdot k_{2}+4 \widetilde{D}_{35} \epsilon_{1} \cdot k_{2} \epsilon_{2} \cdot k_{2} \\
& +\widetilde{D}_{11} \epsilon_{1} \cdot q_{2} \epsilon_{2} \cdot k_{2}-\widetilde{D}_{12} \epsilon_{1} \cdot q_{2} \epsilon_{2} \cdot k_{2}+\widetilde{D}_{13} \epsilon_{1} \cdot q_{2} \epsilon_{2} \cdot k_{2}+4 \widetilde{D}_{23} \epsilon_{1} \cdot q_{2} \epsilon_{2} \cdot k_{2} \\
& +4 \widetilde{D}_{25} \epsilon_{1} \cdot q_{2} \epsilon_{2} \cdot k_{2}-8 \widetilde{D}_{26} \epsilon_{1} \cdot q_{2} \epsilon_{2} \cdot k_{2}-4 \widetilde{D}_{310} \epsilon_{1} \cdot q_{2} \epsilon_{2} \cdot k_{2}+4 \widetilde{D}_{37} \epsilon_{1} \cdot q_{2} \epsilon_{2} \cdot k_{2} \\
& -\widetilde{D}_{11} \epsilon_{1} \cdot k_{2} \epsilon_{2} \cdot q_{1}-7 \widetilde{D}_{12} \epsilon_{1} \cdot k_{2} \epsilon_{2} \cdot q_{1}+7 \widetilde{D}_{13} \epsilon_{1} \cdot k_{2} \epsilon_{2} \cdot q_{1}-4 \widetilde{D}_{22} \epsilon_{1} \cdot k_{2} \epsilon_{2} \cdot q_{1} \\
& -8 \widetilde{D}_{24} \epsilon_{1} \cdot k_{2} \epsilon_{2} \cdot q_{1}+4 \widetilde{D}_{25} \epsilon_{1} \cdot k_{2} \epsilon_{2} \cdot q_{1}+8 \widetilde{D}_{26} \epsilon_{1} \cdot k_{2} \epsilon_{2} \cdot q_{1}+4 \widetilde{D}_{310} \epsilon_{1} \cdot k_{2} \epsilon_{2} \cdot q_{1} \\
& -4 \widetilde{D}_{36} \epsilon_{1} \cdot k_{2} \epsilon_{2} \cdot q_{1}+4 \widetilde{D}_{23} \epsilon_{1} \cdot q_{2} \epsilon_{2} \cdot q_{1}-4 \widetilde{D}_{26} \epsilon_{1} \cdot q_{2} \epsilon_{2} \cdot q_{1}-4 \widetilde{D}_{38} \epsilon_{1} \cdot q_{2} \epsilon_{2} \cdot q_{1}
\end{aligned}
$$




$$
\begin{aligned}
& +4 \widetilde{D}_{39} \epsilon_{1} \cdot q_{2} \epsilon_{2} \cdot q_{1}-\widetilde{D}_{11} \epsilon_{1} \cdot k_{2} \epsilon_{2} \cdot q_{2}-3 \widetilde{D}_{12} \epsilon_{1} \cdot k_{2} \epsilon_{2} \cdot q_{2}+3 \widetilde{D}_{13} \epsilon_{1} \cdot k_{2} \epsilon_{2} \cdot q_{2} \\
& +8 \widetilde{D}_{23} \epsilon_{1} \cdot k_{2} \epsilon_{2} \cdot q_{2}-4 \widetilde{D}_{24} \epsilon_{1} \cdot k_{2} \epsilon_{2} \cdot q_{2}-4 \widetilde{D}_{26} \epsilon_{1} \cdot k_{2} \epsilon_{2} \cdot q_{2}-4 \widetilde{D}_{310} \epsilon_{1} \cdot k_{2} \epsilon_{2} \cdot q_{2} \\
& +4 \widetilde{D}_{37} \epsilon_{1} \cdot k_{2} \epsilon_{2} \cdot q_{2}+4 \widetilde{D}_{23} \epsilon_{1} \cdot q_{2} \epsilon_{2} \cdot q_{2}-4 \widetilde{D}_{26} \epsilon_{1} \cdot q_{2} \epsilon_{2} \cdot q_{2}+4 \widetilde{D}_{33} \epsilon_{1} \cdot q_{2} \epsilon_{2} \cdot q_{2} \\
& -4 \widetilde{D}_{39} \epsilon_{1} \cdot q_{2} \epsilon_{2} \cdot q_{2}-2 \widetilde{D}_{33} \epsilon_{1} \cdot \epsilon_{2} q_{2}^{2}-2 \widetilde{D}_{38} \epsilon_{1} \cdot \epsilon_{2} q_{2}^{2}+4 \widetilde{D}_{39} \epsilon_{1} \cdot \epsilon_{2} q_{2}^{2} \\
& +\frac{1}{2} \widetilde{D}_{11} \epsilon_{1} \cdot \epsilon_{2} s-\frac{1}{2} \widetilde{D}_{12} \epsilon_{1} \cdot \epsilon_{2} s+\frac{1}{2} \widetilde{D}_{13} \epsilon_{1} \cdot \epsilon_{2} s+2 \widetilde{D}_{25} \epsilon_{1} \cdot \epsilon_{2} s \\
& -2 \widetilde{D}_{26} \epsilon_{1} \cdot \epsilon_{2} s-2 \widetilde{D}_{310} \epsilon_{1} \cdot \epsilon_{2} s+2 \widetilde{D}_{37} \epsilon_{1} \cdot \epsilon_{2} s+2 \widetilde{D}_{38} \epsilon_{1} \cdot \epsilon_{2} s \\
& -2 \widetilde{D}_{39} \epsilon_{1} \cdot \epsilon_{2} s+2 \widetilde{D}_{22} \epsilon_{1} \cdot \epsilon_{2} u+2 \widetilde{D}_{23} \epsilon_{1} \cdot \epsilon_{2} u-4 \widetilde{D}_{26} \epsilon_{1} \cdot \epsilon_{2} u \\
& -4 \widetilde{D}_{310} \epsilon_{1} \cdot \epsilon_{2} u+2 \widetilde{D}_{36} \epsilon_{1} \cdot \epsilon_{2} u+2 \widetilde{D}_{37} \epsilon_{1} \cdot \epsilon_{2} u
\end{aligned}
$$

In the above expressions, the finite $\widetilde{D}_{i j}$ functions are obtained by standard PassarinoVeltman recursion relations [28] from the finite parts of the basic scalar integrals. For the virtual corrections considered, only the one-mass box [34, 35], is needed. Specifically, we need the case in which $k_{1}^{2}=k_{2}^{2}=q_{1}^{2}=0$ and $q_{2}^{2} \neq 0$. Here $k_{i}$ and $q_{i}$ with $i=1,2$ are the external four momenta. The one-mass box in the unphysical region, $-s>0,-t>0,-q_{2}^{2}>$ 0 is,

$$
\begin{aligned}
D_{0}\left(k_{2}, q_{2}, q_{1}\right) & =\int \frac{d^{d} k}{i \pi^{2}} \frac{1}{\left[k^{2}\right]\left[\left(k-k_{2}\right)^{2}\right]\left[\left(k-k_{2}-q_{2}\right)^{2}\right]\left[\left(k-k_{2}-q_{2}-q_{1}\right)^{2}\right]} \\
& =\pi^{-\epsilon}\left(\mu^{2}\right)^{-\epsilon} \Gamma(1+\epsilon) \\
& \cdot\left\{\frac{2}{s t} \frac{1}{\epsilon^{2}}+\frac{2}{s t} \frac{1}{\epsilon}\left[\ln \left(\frac{-q_{2}^{2}}{\mu^{2}}\right)-\ln \left(\frac{-s}{\mu^{2}}\right)-\ln \left(\frac{-t}{\mu^{2}}\right)\right]+\widetilde{D}_{0}\left(k_{2}, q_{2}, q_{1}\right)+\mathcal{O}(\epsilon)\right\},
\end{aligned}
$$

where,

$$
\begin{aligned}
\widetilde{D}_{0}\left(k_{2}, q_{2}, q_{1}\right) & =\frac{1}{s t}\left[\ln ^{2}\left(\frac{-s}{\mu^{2}}\right)+\ln ^{2}\left(\frac{-t}{\mu^{2}}\right)-\ln ^{2}\left(\frac{-q_{2}^{2}}{\mu^{2}}\right)\right. \\
& \left.-\ln \left(\frac{-s}{\mu^{2}}\right)+\ln \left(\frac{-t}{\mu^{2}}\right)-2 \operatorname{Li}_{2}\left(1-\frac{q_{2}^{2}}{t}\right)-2 \operatorname{Li}_{2}\left(1-\frac{q_{2}^{2}}{s}\right)-\frac{2 \pi^{2}}{3}\right] .
\end{aligned}
$$

The Mandelstam variables, $s$ and $t$, are defined in Eq. (2.22).

For the present application, the invariant, $q_{2}^{2}$ is always space-like while the Mandelstam invariants, $s$ and $t$, may either be time-like or space-like. Results for physical kinematic regions can be obtained by analytic continuation by replacing the time-like invariant by $t \rightarrow t+i 0^{+}$or $s \rightarrow s+i 0^{+}$.

In addition, to the one-mass box, we also require expressions for the 3-point and 2-point scalar integrals in $d=4-2 \epsilon$ space-time dimensions. For the 3 -point scalar integral,

$$
\begin{aligned}
C_{0}\left(p_{1}^{2}, p_{2}^{2},\left(p_{1}+p_{2}\right)^{2}\right) & =\int \frac{d^{d} k}{i \pi^{2}} \frac{1}{\left[-k^{2}-i 0^{+}\right]\left[-\left(k+p_{1}\right)^{2}-i 0^{+}\right]} \\
& \times \frac{1}{\left[-\left(k+p_{1}+p_{2}\right)^{2}-i 0^{+}\right]},
\end{aligned}
$$

two cases are needed. Here $p_{1}$ and $p_{2}$ represent the external outward flowing four momenta. 
(a). For the two-mass triangle, either, $p_{1}^{2}=0$ or $p_{2}^{2}=0$ and $p_{3}^{2}=\left(p_{1}+p_{2}\right)^{2} \neq 0$.

$$
\begin{aligned}
C_{0}\left(p_{1}^{2}, 0, p_{3}^{2}\right)= & \pi^{-\epsilon}\left(\mu^{2}\right)^{-\epsilon} \Gamma(1+\epsilon) \\
& \cdot\left\{\frac{1}{-p_{3}^{2}-p_{1}^{2}}\left(\ln \left(\frac{-p_{3}^{2}-i 0^{+}}{\mu^{2}}\right)-\ln \left(\frac{-p_{1}^{2}-i 0^{+}}{\mu^{2}}\right)\right) \frac{1}{\epsilon}\right. \\
+ & \left.\widetilde{C}_{0}\left(p_{1}^{2}, p_{3}^{2}\right)+\mathcal{O}(\epsilon)\right\} \\
\widetilde{C}_{0}\left(p_{1}^{2}, p_{3}^{2}\right) & =\frac{1}{2} \frac{1}{-p_{3}^{2}-p_{1}^{2}}\left(\ln ^{2}\left(\frac{-p_{1}^{2}-i 0^{+}}{\mu^{2}}\right)-\ln ^{2}\left(\frac{-p_{3}^{2}-i 0^{+}}{\mu^{2}}\right)\right)
\end{aligned}
$$

(b). For the one-mass triangle, $p_{1}^{2}=p_{2}^{2}=0$ and $p_{3}^{2}=\left(p_{1}+p_{2}\right)^{2} \neq 0$.

$$
\begin{aligned}
C_{0}\left(0,0, p_{3}^{2}\right) & =\pi^{-\epsilon}\left(\mu^{2}\right)^{-\epsilon} \Gamma(1+\epsilon)\left\{\frac{1}{-p_{3}^{2}} \frac{1}{\epsilon^{2}}\right. \\
& \left.-\frac{1}{-p_{3}^{2}} \ln \left(\frac{-p_{3}^{2}-i 0^{+}}{\mu^{2}}\right) \frac{1}{\epsilon}+\widetilde{C}_{0}\left(p_{3}^{2}\right)+\mathcal{O}(\epsilon)\right\} \\
\widetilde{C}_{0}\left(p_{3}^{2}\right) & =-\frac{\pi^{2}}{6} \frac{1}{-p_{3}^{2}}+\frac{1}{-p_{3}^{2}} \frac{1}{2} \ln ^{2}\left(\frac{-p_{3}^{2}-i 0^{+}}{\mu^{2}}\right)
\end{aligned}
$$

The scalar 2-point integral is

$$
\begin{aligned}
B_{0}\left(q^{2}\right) & =\int \frac{d^{d} k}{i \pi^{2}} \frac{1}{\left[-k^{2}-i 0^{+}\right]\left[-(k-q)^{2}-i 0^{+}\right]} \\
& =\pi^{-\epsilon}\left(\mu^{2}\right)^{-\epsilon} \Gamma(1+\epsilon)\left[\frac{1}{\epsilon}+\widetilde{B}_{0}\left(q^{2}\right)+\mathcal{O}(\epsilon)\right]
\end{aligned}
$$

with

$$
\widetilde{B}_{0}\left(q^{2}\right)=2-\ln \frac{-q^{2}-i 0^{+}}{\mu^{2}}
$$

\section{B. Cross section formulas}

In this appendix we give cross section formulas for processes of the type,

$$
g\left(p_{a}\right)+Q\left(p_{b}\right) \rightarrow q\left(p_{1}\right)+\bar{q}\left(p_{3}\right)+Q\left(p_{2}\right)+H(P) .
$$

Results for the crossed process $q\left(p_{a}\right)+Q\left(p_{b}\right) \rightarrow q\left(p_{1}\right)+Q\left(p_{2}\right)+g\left(p_{3}\right)+H(P)$ were already given in Section 2. The finite three parton NLO cross section that results from the cancellation of the $1 / \epsilon^{2}$ and $1 / \epsilon$ poles of the virtual corrections with those of the insertion operator, $\mathbf{I}(\epsilon)$, is

$$
\begin{aligned}
\sigma_{3}^{N L O}(g Q \rightarrow q \bar{q} Q H)= & \int_{0}^{1} d x_{a} \int_{0}^{1} d x_{b} f_{g / p}\left(x_{a}, \mu_{F}\right) f_{Q / p}\left(x_{b}, \mu_{F}\right) \\
\times & \frac{1}{2 \hat{s}} d \Phi_{4}\left(p_{a}, p_{b}\right) F_{J}^{(3)}\left(p_{1}, p_{2}, p_{3}, P ; p_{a}, p_{b}\right) \\
& \cdot \sum_{\text {colors }}\left\{\left|\mathcal{M}_{3}\left(1_{q}, 2_{Q}, 3_{\bar{q}}, a_{g}, b_{Q}\right)\right|^{2}\left(1+\frac{\alpha_{s}\left(\mu^{2}\right)}{2 \pi}\left(K_{\text {born }}+F\left(s_{13}, s_{a 3}, s_{a 1}\right)\right)\right)\right. \\
+ & \left.2 \operatorname{Re}\left[\widetilde{\mathcal{M}}_{3}^{\text {virt }}\left(1_{q}, 2_{Q}, 3_{\bar{q}}, a_{g}, b_{Q}\right) \mathcal{M}_{3}^{*}\left(1_{q}, 2_{Q}, 3_{\bar{q}}, a_{g}, b_{Q}\right)\right]\right\}
\end{aligned}
$$


with

$$
\widetilde{\mathcal{M}}_{3}^{\text {virt }}\left(1_{q}, 2_{Q}, 3_{\bar{q}}, a_{g}, b_{Q}\right)=t^{c_{a}} \delta_{i_{1} i_{3}} \widetilde{\mathcal{M}}_{V}\left(p_{1},-p_{a}, p_{a 13} ; \epsilon_{a}, h\left(p_{b} \tau_{b}, p_{2} \tau_{2}\right)\right)
$$

The Born level matrix element squared is

$$
\overline{\sum_{\text {colors }}}\left|\mathcal{M}_{3}\left(1_{q}, 2_{Q}, 3_{\bar{q}}, a_{g}, b_{Q}\right)\right|^{2}=\frac{C_{F} N}{N^{2}-1}\left|\mathcal{A}_{3}\left(1_{q}, a_{g}, 3_{\bar{q}} ; 2_{Q}, b_{Q}\right)\right|^{2}
$$

with

$$
\mathcal{A}_{3}\left(1_{q}, a_{g}, 3_{\bar{q}} ; 2_{Q}, b_{Q}\right)=\mathcal{M}_{B}\left(p_{1},-p_{a}, p_{a 13} ; \epsilon_{a}, h\left(p_{b} \tau_{b}, p_{2} \tau_{2}\right)\right) .
$$

The finite collinear contribution is

$$
\begin{aligned}
\sigma_{3, \mathrm{col}}^{N L O}(g Q \rightarrow q \bar{q} Q H) & =\int_{0}^{1} d x_{a} \int_{0}^{1} d x_{b} \frac{1}{2 \hat{s}} d \Phi_{4}\left(p_{a}, p_{b}\right) F_{J}^{(3)}\left(p_{1}, p_{2}, p_{3} ; p_{a}, p_{b}\right) \\
& \cdot\left\{f_{g / p}\left(x_{a} ; \mu_{F}\right) f_{Q / p}^{2, b}\left(x_{b} ; \mu_{F}, \mu_{R}\right)\right. \\
+ & \left.\frac{1}{2}\left(f_{g / p}^{1, a}\left(x_{a} ; \mu_{F}, \mu_{R}\right)+f_{g / p}^{3, a}\left(x_{a} ; \mu_{F}, \mu_{R}\right)\right) f_{Q / p}\left(x_{b} ; \mu_{F}\right)\right\} \\
& \cdot \frac{C_{F} N}{N^{2}-1}\left|\mathcal{A}_{3}\left(1_{q}, a_{g}, 3_{\bar{q}} ; 2_{Q}, b_{Q}\right)\right|^{2}
\end{aligned}
$$

with

$$
\begin{aligned}
f_{g / p}^{i, a}\left(x_{a} ; \mu_{F}, \mu_{R}\right) & =\frac{\alpha_{s}\left(\mu_{R}\right)}{2 \pi} \int_{x_{a}}^{1} \frac{d z}{z}\left\{\sum_{q}\left[f_{q / p}\left(\frac{x_{a}}{z} ; \mu_{F}\right)+f_{\bar{q} / p}\left(\frac{x_{a}}{z} ; \mu_{F}\right)\right] A_{q g}^{i, a}(z)\right. \\
& +\left[f_{g / p}\left(\frac{x_{a}}{z} ; \mu_{F}\right)-z f_{g / p}\left(x_{a} ; \mu_{F}\right)\right] B_{g g}^{i, a}(z) \\
& \left.+f_{g / p}\left(\frac{x_{a}}{z} ; \mu_{F}\right) C_{g g}^{i, a}(z)\right\}+\frac{\alpha_{s}\left(\mu_{R}\right)}{2 \pi} f_{g / p}\left(x_{a} ; \mu_{F}\right) D_{g g}^{i, a}\left(x_{a}\right)
\end{aligned}
$$

with kernels,

$$
\begin{aligned}
A_{q g}^{i, a}(z) & =C_{F}\left[\frac{1+(1-z)^{2}}{z} \ln \frac{2 p_{a} p_{i}(1-z)}{\mu_{F}^{2} z}+z\right] \\
B_{g g}^{i, a}(z) & =C_{A}\left[\frac{2}{1-z} \ln \frac{2 p_{a} p_{i}(1-z)}{\mu_{F}^{2}}-\frac{3}{2} \frac{1}{1-z}\right], \\
C_{g g}^{i, a}(z) & =2 C_{A}\left[\left(\frac{1-z}{z}-1+z(1-z)\right) \ln \frac{2 p_{a} p_{i}(1-z)}{\mu_{F}^{2} z}-\frac{1}{1-z} \ln z\right], \\
D_{g g}^{i, a}(x) & =2 C_{A} \ln (1-x) \ln \frac{2 p_{a} p_{i}}{\mu_{F}^{2}}+\gamma_{g} \ln \frac{2 p_{a} p_{i}}{\mu_{F}^{2}} \\
& +C_{A}\left(\frac{2 \pi^{2}}{3}-\frac{50}{9}+\ln ^{2}(1-x)\right) \\
& +\frac{16}{9} T_{R} N_{f}-\frac{3}{2} C_{A}-\frac{3}{2} C_{A} \ln (1-x) .
\end{aligned}
$$




\section{References}

[1] ATLAS Collaboration, ATLAS TDR, ATLAS Detector and Physics Performance Technical Design Report, Report No. CERN/LHCC/99-15 (1999); E. Richter-Was and M. Sapinski, "Search for the SM and MSSM Higgs boson in $t \bar{t} H, H \rightarrow b \bar{b}$ channel," Acta Phys. Polon. B30 (1999) 1001; B. P. Kersevan and E. Richter-Was, "What is the $W b \bar{b}, Z b \bar{b}$ or $t \bar{t} b \bar{b}$ irreducible background to the light-Higgs boson searches at LHC? "Eur. Phys. J. C 25 (2002) 379 hep-ph/0203148.

[2] G. L. Bayatian et al., CMS Technical Proposal, Report No. CERN/LHCC/94-38x (1994); D. Denegri, "Prospects for Higgs (SM and MSSM) searches at LHC," talk in the Circle Line Tour Series, Fermilab, October 1999, (http://www-theory.fnal.gov/CircleLine/DanielBG.html); R. Kinnunen and D. Denegri, "Expected SM/SUSY Higgs observability in CMS," CMS Note No. 1997/057; R. Kinnunen and A. Nikitenko, "Study of $H_{S U S Y} \rightarrow \tau \tau \rightarrow l^{ \pm}+h^{\mp}+E_{t}^{m i s s}$ in CMS," Report No. CMS TN/97-106; R. Kinnunen and D. Denegri, "The $H_{S U S Y} \rightarrow \tau \tau \rightarrow h^{ \pm}+h^{\mp}+X$ channel, its advantages and potential instrumental drawbacks," hep-ph/9907291; V. Drollinger, T. Müller and D. Denegri, "Searching for Higgs Bosons in Association with Top Quark Pairs in the $H \rightarrow b \bar{b}$ Decay Mode," hep-ph/0111312

[3] D. L. Rainwater, PhD thesis, "Intermediate-mass Higgs searches in weak-boson fusion," hep-ph/9908378.

[4] D. Zeppenfeld, R. Kinnunen, A. Nikitenko and E. Richter-Was, "Measuring Higgs boson couplings at the LHC," Phys. Rev. D 62 (2000) 013009 hep-ph/0002036; D. Zeppenfeld, "Higgs couplings at the LHC," in Proc. of the APS/DPF/DPB Summer Study on the Future of Particle Physics, Snowmass, 2001 edited by N. Graf, eConf C010630, (2001) 123 hep-ph/0203123; A. Belyaev and L. Reina, " $p p \rightarrow t \bar{t} H, H \rightarrow \tau^{+} \tau^{-}$: Toward a model independent determination of the Higgs boson couplings at the LHC," J. High Energy Phys. 08 (2002) 041 hep-ph/0205270; M. Dührssen et al., S. Heinemeyer, H. Logan, D. Rainwater, G. Weiglein and D. Zeppenfeld, "Extracting Higgs boson couplings from LHC data," Phys. Rev. D 70 (2004) 113009 hep-ph/0406323.

[5] N. Kauer, T. Plehn, D. L. Rainwater and D. Zeppenfeld, "H $\rightarrow \mathrm{W}$ W as the discovery mode for a light Higgs boson," Phys. Lett. B 503 (2001) 113 hep-ph/0012351.

[6] D. Rainwater, D. Zeppenfeld and K. Hagiwara, "Searching for $H \rightarrow \tau \tau$ in weak boson fusion at the LHC," Phys. Rev. D 59 (1999) 014037 hep-ph/9808468]; T. Plehn, D. Rainwater and D. Zeppenfeld, "Method for identifying $H \rightarrow \tau \tau \rightarrow e^{ \pm} \mu^{\mp} p_{T}$ at the CERN LHC," Phys. Rev. D 61 (2000) 093005 hep-ph/9911385; S. Asai et al., "Prospects for the Search of a Standard Model Higgs Boson in ATLAS using Vector Boson Fusion," Eur. Phys. J. C 32 (2004) 19.

[7] C. M. Buttar, R. S. Harper and K. Jakobs, "Weak boson fusion $H \rightarrow W W^{*} \rightarrow l^{+} l^{-} p_{T}$ miss as a search mode for an intermediate mass SM Higgs boson at ATLAS," ATL-PHYS-2002-033; K. Cranmer et al., "Search for Higgs Bosons Decay $H \rightarrow W^{+} W^{-} \rightarrow l^{+} l^{-} \not p_{T}$ for $115<M_{H}<130 \mathrm{GeV}$ using Vector Boson Fusion," ATL-PHYS-2003-002; K. Cranmer et al., "Neural Networks Based Search for Higgs Bosons Produced via VBF with $H \rightarrow W^{+} W^{-} \rightarrow l^{+} l^{-} \not p_{T}$ for $115<M_{H}<130 \mathrm{GeV}$," ATL-PHYS-2003-007.

[8] D. L. Rainwater and D. Zeppenfeld, "Observing $H \rightarrow W^{(*)} W^{(*)} \rightarrow e^{ \pm} \mu^{\mp} p_{T}$ in weak boson fusion with dual forward jet tagging at the CERN LHC," Phys. Rev. D 60 (1999) 113004 [Erratum-ibid. D 61, (2000) 099901] hep-ph/9906218]. 
[9] D. Rainwater and D. Zeppenfeld, "Searching for $H \rightarrow \gamma \gamma$ in weak boson fusion at the LHC," J. High Energy Phys. 12 (1997) 005 hep-ph/9712271. K. Cranmer, B. Mellado, W. Quayle and S. L. Wu, "Search for Higgs bosons decay $H \rightarrow \gamma \gamma$ using vector boson fusion," hep-ph/0401088.

[10] O. J. Eboli and D. Zeppenfeld, "Observing an invisible Higgs boson," Phys. Lett. B 495 (2000) 147 hep-ph/0009158; B. Di Girolamo, A. Nikitenko, L. Neukermans, K. Mazumdar and D. Zeppenfeld, "Experimental observation of an invisible Higgs boson at LHC," hep-ph/0203056.

[11] V. Del Duca, A. Frizzo and F. Maltoni, "Higgs boson production in association with three jets," J. High Energy Phys. 05 (2004) 064 hep-ph/0404013.

[12] J. D. Bjorken, "Rapidity gaps and jets as a new physics signature in very high-energy hadron hadron collisions," Phys. Rev. D 47 (1993) 101

[13] D. L. Rainwater, R. Szalapski and D. Zeppenfeld, "Probing color-singlet exchange in Z + 2-jet events at the LHC," Phys. Rev. D 54 (1996) 6680 hep-ph/9605444.

[14] V. D. Barger, R. J. N. Phillips and D. Zeppenfeld, "Mini - jet veto: A Tool for the heavy Higgs search at the LHC," Phys. Lett. B 346 (1995) 106 hep-ph/9412276.

[15] V. D. Barger, K. M. Cheung, T. Han and D. Zeppenfeld, "Finding the leptonic W W decay mode of a heavy Higgs boson at hadron supercolliders," Phys. Rev. D 48 (1993) 5433 hep-ph/9305277.

[16] V. D. Barger, K. M. Cheung, T. Han and D. Zeppenfeld, "Single forward jet tagging and central jet vetoing to identify the leptonic W W decay mode of a heavy Higgs boson," Phys. Rev. D 44 (1991) 2701 [Erratum-ibid. D 48, (1993) 5444].

[17] A. Duff and D. Zeppenfeld, "Heavy Higgs boson production in association with three jets at hadron supercolliders," Phys. Rev. D 50 (1994) 3204 hep-ph/9312357.

[18] T. Han, G. Valencia and S. Willenbrock, "Structure function approach to vector boson scattering in p p collisions," Phys. Rev. Lett. 69 (1992) 3274 hep-ph/9206246.

[19] T. Figy, C. Oleari and D. Zeppenfeld, "Next-to-leading order jet distributions for Higgs boson production via weak-boson fusion," Phys. Rev. D 68 (2003) 073005 hep-ph/0306109.

[20] E. L. Berger and J. Campbell, "Higgs boson production in weak boson fusion at next-to-leading order," Phys. Rev. D 70 (2004) 073011 hep-ph/0403194.

[21] C. Georg, diploma thesis, http://www-itp.particle.uni-karlsruhe.de/diplomatheses.de.shtml; J. R. Andersen and J. M. Smillie, "QCD and electroweak interference in Higgs production by gauge boson fusion," Phys. Rev. D 75 (2007) 037301 [arXiv:hep-ph/0611281].

[22] M. Ciccolini, A. Denner and S. Dittmaier, "Strong and electroweak corrections to the production of Higgs+2jets via weak interactions at the LHC," Phys. Rev. Lett. 99 (2007) 161803 arXiv:0707.0381; "Electroweak and QCD corrections to Higgs production via vector-boson fusion at the LHC," arXiv:0710.4749; J. R. Andersen, T. Binoth, G. Heinrich and J. M. Smillie, "Loop induced interference effects in Higgs Boson plus two jet production at the LHC," arXiv:0709.3513.

[23] K. Hagiwara and D. Zeppenfeld, "Helicity Amplitudes for Heavy Lepton Production in $e^{+} e^{-}$ Annihilation," Nucl. Phys. B 274 (1986) 1;

K. Hagiwara and D. Zeppenfeld, "Amplitudes for Multiparton Processes Involving a Current at $e^{+} e^{-}, e^{ \pm} p$, and Hadron Colliders," Nucl. Phys. B 313 (1989) 560. 
[24] S. Dittmaier, "Separation of soft and collinear singularities from one-loop N-point integrals," Nucl. Phys. B 675 (2003) 447 hep-ph/0308246.

[25] T. Stelzer and W. F. Long, "Automatic generation of tree level helicity amplitudes," Comput. Phys. Commun. 81 (1994) 357 hep-ph/9401258;

F. Maltoni and T. Stelzer, "MadEvent: Automatic event generation with MadGraph," J. High Energy Phys. 02 (2003) 027 hep-ph/0208156.

[26] S. Catani and M. H. Seymour, "A general algorithm for calculating jet cross sections in NLO QCD," Nucl. Phys. B 485 (1997) 291 [Erratum-ibid. B510, 503 (1997)] hep-ph/9605323.

[27] C. Oleari and D. Zeppenfeld, "QCD corrections to electroweak $\ell \nu_{\ell} j j$ and $\ell^{+} \ell^{-} j j$ production," Phys. Rev. D 69 (2004) 093004 hep-ph/0310156.

[28] G. Passarino and M. J. Veltman, "One Loop Corrections For E+ E- Annihilation Into Mu+ Mu- In The Weinberg Model," Nucl. Phys. B 160 (1979) 151

[29] Z. Nagy, "Next-to-leading order calculation of three-jet observables in hadron hadron collision," Phys. Rev. D 68 (2003) 094002 hep-ph/0307268.

[30] J. Pumplin, D. R. Stump, J. Huston, H. L. Lai, P. Nadolsky and W. K. Tung, "New generation of parton distributions with Uncertainties from Global QCD Analysis ," J. High Energy Phys. 07 (2002) 012 hep-ph/0201195.

[31] S. Catani, Yu. L. Dokshitzer and B. R. Webber, "The K-perpendicular clustering algorithm for jets in deep inelastic scattering and hadron collisions," Phys. Lett. B 285 (1992) 291;

S. Catani, Yu. L. Dokshitzer, M. H. Seymour and B. R. Webber, "Longitudinally invariant K(t) clustering algorithms for hadron hadron collisions," Nucl. Phys. B 406 (1993) 187; S. D. Ellis and D. E. Soper, "Successive combination jet algorithm for hadron collision," Phys. Rev. D 48 (1993) 3160

[32] G. C. Blazey et al., "Run II jet physics," hep-ex/0005012.

[33] T. Brodkorb and J. G. Körner, "Lepton - hadron correlations to $\mathrm{O}($ alpha-s**2) in $(2+1)$ jet production at electron - proton colliders," Z. Physik C 54 (1992) 519 D. Graudenz, "Next-to-leading order QCD corrections to jet cross-sections and jet rates in deeply inelastic electron proton scattering," Phys. Rev. D 49 (1994) 3291 hep-ph/9307311; D. Graudenz, "DISASTER++ version 1.0," hep-ph/9710244; E. Mirkes and D. Zeppenfeld, "Dijet Production at HERA in Next-to-Leading Order," Phys. Lett. B 380 (1996) 205 hep-ph/9511448; S. Catani and M. H. Seymour, "QCD jet calculations in DIS based on the subtraction method and dipole formalism," hep-ph/9609237.

[34] Z. Bern, L. J. Dixon and D. A. Kosower, "Dimensionally regulated pentagon integrals," Nucl. Phys. B 412 (1994) 751 hep-ph/9306240].

[35] S. Papadopoulos, A. P. Contogouris and J. Ralston, "Calculation of Box Graph with Lightlike Particles," Phys. Rev. D 25 (1982) 2218. 